JOURNAL OF

SYMPLECTIC GEOMETRY

Volume 10, Number 2, 247-324, 2012

\title{
A PRODUCT FORMULA FOR GROMOV-WITTEN INVARIANTS
}

\author{
Clément Hyvrier
}

We establish a product formula for Gromov-Witten invariants for closed relatively semi-positive Hamiltonian fibrations, with connected fiber, and over any connected symplectic base. Furthermore, we show that the fibration projection induces a locally trivial (orbi-)fibration map from the moduli space of pseudo-holomorphic maps with marked points in the total space of the Hamiltonian fibration to the corresponding moduli space of pseudo-holomorphic maps with marked points in the base. We use this induced map to recover the product formula by means of integration. Finally, we give applications to $c$-splitting and symplectic uniruledness.

\section{Contents}

1. Introduction

2. The framework

2.1. Hamiltonian connections and coupling form

3. The Cauchy-Riemann problem in Hamiltonian fibrations

3.1. Splitting of Fredholm systems

3.2. Splitting of Fredholm systems for Hamiltonian fibrations

4. Structure theorems

\subsection{Stable holomorphic maps and Hamiltonian} fibrations 
4.1.2. Moduli space of stable maps 275

4.1.3. Energy identities $\quad 276$

4.1.4. Forgetful maps and Hamiltonian fibrations $\quad 277$

4.2. Transversality on every strata 278

4.2.1. The non-fibered case. 278

4.2.2. The Hamiltonian fibration case $\quad 280$

4.2.3. Cobordisms 286

5. The product formula $\quad 286$

5.1. Evaluation maps as pseudo-cycles 289

5.2. Proof of Theorem A 290

6. Gluing and fibration of moduli spaces $\quad 292$

6.1. Gluing in the non-fibered case: $B=p t$

6.1.1. Gluing for nodal curves $\quad 294$

6.1.2. Gluing stable components 295

6.1.3. Gluing unstable components 303

6.2. Gluing for general $B \quad 307$

6.2.1. Pregluing $\quad 307$

6.2.2. Right inverses $\quad 308$

6.2.3. Gluing stable components $\quad 312$

6.2.4. Gluing: the unstable case. 313

6.2.5. Gluing maps between strata 317

6.3. Fibration of moduli spaces: proof of Theorem C $\quad 318$

6.3.1. Charts data and admissible gluing maps $\quad 318$

6.3.2. Structure of orbi-bundle $\quad 320$

References

323

\section{Introduction}

We consider rational Gromov-Witten invariants ( $G W$-invariants) of Hamiltonian fibrations with closed connected fiber over any closed connected symplectic base. In a symplectic manifold $(X, \omega)$ with $\omega$-tame almost complex structure $J, G W$-invariants are given by counting the (algebraic) number of unparameterized $J$-(pseudo)-holomorphic genus 0 maps with $l$ distinct marked points, representing a fixed spherical homology class $A \in H_{2}(X, \mathbb{Z})$, and intersecting transversally $l$ given cycles of $X$ at the marked points. Roughly speaking, if $\mathcal{M}_{0, l}(X, A, J)$ denotes the moduli space of unparameterized (genus 0 ) $J$-holomorphic maps with $l$ markings, $\left(u, x_{1}, \ldots, x_{l}\right)$, representing the class $A$, and if $M_{1}, \ldots, M_{l}$ are cycles in $X$ representing given classes $c_{1}, \ldots, c_{l} \in H_{*}(X, \mathbb{Q})$, then these invariants can be seen as the values 
of the homomorphism:

$$
\begin{aligned}
\langle\rangle_{0, l, A}^{X}:\left(H_{*}(X, \mathbb{Q})\right)^{\otimes l} & \rightarrow \mathbb{Q} \\
c_{1} \otimes \cdots \otimes c_{l} & \mapsto e v_{l}^{X} \cdot\left(M_{1} \times \cdots \times M_{l}\right),
\end{aligned}
$$

the intersection pairing - being taken with respect to the evaluation at the marked points:

$$
e v_{l, J}^{X}: \mathcal{M}_{0, l}(X, A, J) \rightarrow X^{l}, \quad\left(u, x_{1}, \ldots, x_{l}\right) \mapsto\left(u\left(x_{1}\right), \ldots, u\left(x_{n}\right)\right) .
$$

It is well known that when the symplectic manifold is semi-positive the $G W$ invariants are generically well-defined $\mathbb{Z}$-valued invariants of the symplectic manifold, and the classes $c_{i}$ can be chosen in $H_{*}(X)$ the singular homology of $X$ modulo torsion (see $[\mathbf{1 7}, \mathbf{2 1}]$ ).

In the case where the Hamiltonian fibration is a product of two symplectic manifolds, Ruan and Tian [21], and shortly after Kontsevich and Manin [9], showed that when both the base and the fiber are semi-positive, the $G W$ invariants of the total space are given by products of related $G W$-invariants in the base and in the fiber, giving rise to splitting of the quantum product. A priori, we cannot expect for such a splitting to hold in the non-trivial case as even the cup product may not split. Nevertheless, one may still ask about the algebraic relations that can be established out of the invariants of the base, the fiber and the total space. We give such a relation under some assumptions, in particular when the reference fiber of the Hamiltonian fibration is semi-positive relative to the total space. We now explain in detail the setting, our main results and some consequences.

Hamiltonian fibrations. By definition, a symplectic fibration is a smooth locally trivial fibration $\pi: P \rightarrow B$ with symplectic reference fiber $\left(F^{2 n_{\mathrm{F}}}, \omega\right)$ whose structure group lies in the group of symplectic diffeomorphisms of the fiber, denoted $\operatorname{Symp}(F, \omega)$. It follows that each fiber $F_{b}:=\pi^{-1}(b)$ is naturally equipped with a symplectic form $\omega_{b}$. A symplectic fibration is Hamiltonian if the structure group can be reduced to the group $\operatorname{Ham}(F, \omega)$ of Hamiltonian diffeomorphisms. Extending results of GuilleminLerman-Sternberg for Symplectic fibrations with simply connected fibers ([5], Theorem 1.4.1), McDuff and Salamon showed the following criterion for Hamiltonian fibrations:

Theorem 1.1 ([16], Theorem 6.21). A Symplectic fibration as above is Hamiltonian if and only if:

$\left(H_{1}\right): P$ is symplectically trivial over the 1-skeleton $B_{1}$ of $B$;

$\left(H_{2}\right)$ : there exists a connection $H_{o r} \subset T P$ with holonomy in $\operatorname{Ham}(F, \omega)$, induced by a unique closed 2-form $\tau \in \Omega^{2}(P)$ extending the family $\left\{\omega_{b}\right\}_{b \in B}$, and such that the integration of $\tau^{n_{F}+1}$ along the fibers of $\pi$ vanishes. 
The closed 2-form is usually referred to as the coupling form. Since $B$ is assumed to be closed and symplectic, the existence of such a form is sufficient to give $P$ a symplectic structure compatible with the family $\left\{\omega_{b}\right\}_{b \in B}$ by considering the form

$$
\omega_{P, \kappa}:=\tau+\kappa \pi^{*} \omega_{B},
$$

where $\kappa>0$ is a real number chosen large enough, so that $\omega_{P, \kappa}$ is nondegenerate. A simple example of such fibration is the trivial product of the base with the fiber. A less trivial class of examples is that of Hamiltonian fibrations over $S^{2}$. These latter fibrations correspond (up to isomorphism) to homotopy classes of loops in $\operatorname{Ham}(F, \omega)$. This direct connection with the fundamental group of $\operatorname{Ham}(F, \omega)$ makes these objects particularly interesting from the point of view of symplectic topology as pointed out by Seidel $[\mathbf{2 2}]$.

Product formula. Let $\sigma \in H_{2}(P ; \mathbb{Z})$ be a spherical homology class such that $\sigma_{B}:=\pi_{*} \sigma$ is non-zero. Also, consider classes in $H_{*}(P)$ that are either lying in the image of $\iota_{\mathrm{F}}^{P}: H_{*}(F) \rightarrow H_{*}(P)$ or in the image of the "shriek" map

$$
\pi_{!}: H_{*}(B) \rightarrow H_{2 n_{\mathrm{F}}+*}(P), \quad \alpha \mapsto P D_{P}^{-1} \pi^{*} P D_{B}(\alpha) .
$$

Explicitly, we will consider classes $c_{i}^{B} \in H_{*}(B), c_{i}^{F} \in H_{*}(F)$ and $c_{i}^{P} \in$ $H_{*}(P), i=1, \ldots, l$, such that the following conditions are satisfied for some integer $0 \leq m \leq l$ :

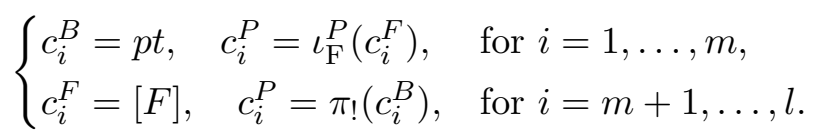

In this paper, we provide a product-type formula giving the $G W$-invariant $\left\langle c_{1}^{P}, \ldots, c_{l}^{P}\right\rangle_{0, l, \sigma}^{P}$ of $P$ as the product of $G W$-invariants $\left\langle c_{1}^{B}, \ldots, c_{l}^{B}\right\rangle_{0, l, \sigma_{B}}^{B}$ of $B$ with a term involving $G W$-invariants of a Hamiltonian fibration over $S^{2}$. Such a product formula was suggested in [10].

For this purpose, we equip $P$ with an (almost) complex structure $J_{P}$ compatible with $\pi$ and a Hamiltonian connection $\tau$, which means that $J_{P}$ projects to an $\omega_{B}$-tame almost complex structure $J_{B}$ via $d \pi, J_{P}$ preserves the horizontal distribution $\mathrm{Hor}_{\tau}$, and $J_{P}$ restricts to an $\omega_{b}$-tame almost complex structure $J_{b}$ on $T F_{b}$. For simplicity, we will say that such $J_{P}$ is fibered. It follows from this choice of almost complex structure that $\pi$ induces a map between moduli spaces of unparameterized pseudo-holomorphic maps:

$$
\bar{\pi}: \mathcal{M}_{0, l}\left(P, \sigma, J_{P}\right) \rightarrow \mathcal{M}_{0, l}\left(B, \sigma_{B}, J_{B}\right), \quad(u, \mathbf{x}) \mapsto(\pi(u), \mathbf{x}) .
$$

In fact, the third term in the product formula, that we have not defined yet, is related to the fibers of this map. Let us describe the fiber of $\bar{\pi}$ over $\left(u_{B}, \mathbf{x}\right)$. Let $C$ denote the image of the $J_{B}$-holomorphic map $u_{B}$ representing $\sigma_{B}$, and let $P_{C}$ denote the restriction of $P$ along $C$. Then, $P_{C}$ is a Hamiltonian 
fibration over $S^{2}$ with coupling form given by the pull-back of $\tau$ under the natural inclusion $\iota_{P_{C}}^{P}: P_{C} \hookrightarrow P$. Moreover, the restriction of $J_{P}$ to $P_{C}$ defines a fibered almost complex structure $J_{C}$ on $P_{C}$. Then $\bar{\pi}^{-1}\left(u_{B}, \mathbf{x}\right)$ consists of unparameterized $J_{C}$-holomorphic maps in $P_{C}$ with fixed marked points $\mathbf{x}$ representing classes belonging to the set

$$
B_{\sigma}:=\left\{\sigma^{\prime} \in H_{2}\left(P_{C} ; \mathbb{Z}\right) \mid \iota_{P_{C}}^{P} \sigma^{\prime}=\sigma\right\},
$$

where $\iota_{P_{C}}^{P}$ stands for the induced map in homology. Roughly speaking, the third term in the product formula is actually a sum over $B_{\sigma}$ of $G W$-invariants of $P_{C}$ involving the classes $\iota_{\mathrm{F}}^{P_{C}}\left(c_{i}^{F}\right)$, where $C$ is the image of some map counted in $\left\langle c_{1}^{B}, \ldots, c_{l}^{B}\right\rangle_{0, l, \sigma_{B}}^{B}$, and where $\iota_{\mathrm{F}}^{P_{C}}$ denotes the map in homology induced from the natural inclusion of $F$ into $P_{C}$. We will provide two proofs for this formula.

The first proof rests on a transversality result for fibered almost complex structures. The formula is then obtained by explicitly identifying the curves entering the definition of the involved $G W$-invariants. To achieve transversality we will assume that the fiber is semi-positive relative to the total space: let $c^{v}$ denote the first Chern class of the vertical subbundle ker $d \pi \subset T P$ (induced by a family of $\omega_{b}$-tame almost complex structures on $F_{b}$ ); we say that $(F, \omega)$ is semi-positive relative to $P$ if and only if

$(\star \star) \quad \forall A \in \pi_{2}(F): \omega(A)>0, \quad c^{v}(A) \geq 3-1 / 2 \operatorname{dim} P \Longrightarrow c^{v}(A) \geq 0$,

where $A$ is seen as an element in $\pi_{2}(P)$ via the natural inclusion $\iota_{\mathrm{F}}^{P}: F \hookrightarrow P$. Note that $P$ need not be semi-positive, but the fiber must be. We also require that $\sigma_{B}$ only admits irreducible effective decompositions for some $J_{B}$ : this means that any stable $J_{B}$-holomorphic map with $l$-marked points representing $\sigma_{B}$ is simple, i.e., admits no multiply covered component or any two components having the same non-constant image in $B$. This is in particular realized by effective primitive classes (see Section 4.1.2).

Theorem A. Let $\pi: P \rightarrow B$ be a Hamiltonian fibration with semi-positive fiber $(F, \omega)$ relatively to $P$. Let $\sigma \in H_{2}(P, \mathbb{Z})$ and suppose $\sigma_{B}:=\pi_{*}(\sigma) \neq 0$ only admits irreducible effective decompositions for some $J_{B}$. Let $c_{i}^{P}, c_{i}^{B}, c_{i}^{F}$ be as in $(\star)$. Then, for a generic fibered complex structure the following equation holds:

$$
\left\langle c_{1}^{P}, \ldots, c_{l}^{P}\right\rangle_{0, l, \sigma}^{P}=\left\langle c_{1}^{B}, \ldots, c_{l}^{B}\right\rangle_{0, l, \sigma_{B}}^{B} \cdot \sum_{\sigma^{\prime} \in B_{\sigma}}\left\langle\iota_{\mathrm{F}}^{P_{C}}\left(c_{1}^{F}\right), \ldots, \iota_{\mathrm{F}}^{P_{C}}\left(c_{l}^{F}\right)\right\rangle_{0, l, \sigma^{\prime}}^{P_{C}},
$$

where $C$ is a curve counted in $\left\langle c_{1}^{B}, \ldots, c_{l}^{B}\right\rangle_{0, l, \sigma_{B}}^{B}$.

By Gromov's compactness the above sum is finite. It is even possible to simplify the expression of the formula by considering equivalence classes on the preimage $\pi_{*}^{-1}\left(\sigma_{B}\right) \subset H_{2}(P, \mathbb{Z})$. More precisely, we say that 
$\sigma_{1}, \sigma_{2} \in \pi_{*}^{-1}\left(\sigma_{B}\right)$ are equivalent if and only if

$$
\tau\left(\sigma_{1}-\sigma_{2}\right)=0=c^{v}\left(\sigma_{1}-\sigma_{2}\right) .
$$

Let $[\sigma]_{\sigma_{B}}$ denote the equivalence class of $\sigma$ in the product formula. Note that, under the pull-back by $\iota_{P_{C}}^{P}$, any element in $\pi_{*}^{-1}\left(\sigma_{B}\right)$ defines a section class of $P_{C}$, i.e., a class projecting on $\left[S^{2}\right]$ under $\pi_{*}$. Moreover, it is not hard to see that the preimage of $[\sigma]_{\sigma_{B}}$ under $\iota_{P_{C}}^{P}$ gives rise to an equivalence class of section classes in $P_{C}$. If $\sigma_{C}$ denotes this equivalence class then the sum in the product formula disappears:

$$
\left\langle c_{1}^{P}, \ldots, c_{l}^{P}\right\rangle_{0, l,[\sigma]_{\sigma_{B}}^{P}}^{P}=\left\langle c_{1}^{B}, \ldots, c_{l}^{B}\right\rangle_{0, l, \sigma_{B}}^{B} \cdot\left\langle\iota_{\mathrm{F}}^{P_{C}}\left(c_{1}^{F}\right), \ldots, \iota_{\mathrm{F}}^{P_{C}}\left(c_{l}^{F}\right)\right\rangle_{0, l, \sigma_{C}}^{P_{C}} .
$$

Also, let us mention that the case $\sigma_{B}=0$ leads to the Parametric $G W$ invariants, which are well known $[\mathbf{2}, \mathbf{1 2}]$.

An important issue in the proof of Theorem A is establishing that the $G W$-invariants involved are generically and simultaneously well-defined. More precisely, the problem is to realize generically both moduli spaces in (1.1) as smooth oriented manifolds together with preserving $\bar{\pi}$. In the general context of a symplectic manifold $(X, \omega)$ it is well known that the irreducible component $\mathcal{M}_{0, l}^{*}(X, A, J)$ of $\mathcal{M}_{0, l}(X, A, J)$ consisting of simple maps is actually an oriented open manifold of finite dimension for a generic choice of $\omega$-tame structure $J[\mathbf{1 7}, \mathbf{1 9}]$. Here we cannot directly apply this result since tame almost complex structures of the total space do not coincide with fibered almost complex structures. Nevertheless, generalizing a result of McDuff and Salamon in the case of Hamiltonian fibrations over Riemann surfaces ([17], Theorem 8.3.1) we prove that:

Theorem B. Suppose $\sigma_{B} \neq 0$. There exists a second category subset, $\mathcal{J}_{P, \mathrm{reg}}$, of fibered almost complex structures such that for every $J_{P} \in \mathcal{J}_{P \text {,reg }}$

(1) the subset $\mathcal{M}_{0, l}^{* *}\left(P, \sigma, J_{P}\right)$ of $\mathcal{M}_{0, l}\left(P, \sigma, J_{P}\right)$ consisting of simple maps that project to simple maps under $\bar{\pi}$, and the moduli space $\mathcal{M}_{0, l}^{*}\left(B, \sigma_{B}\right.$, $\left.J_{B}\right)$, are open-oriented manifolds.

(2) for any countable set $Z$ of elements in $\mathcal{M}_{0, l}^{*}\left(B, \sigma_{B}, J_{B}\right)$, for every $u \in Z$, the preimage $\bar{\pi}^{-1}(u)$ is an open oriented manifold.

The dimensions of the manifolds in (1) are respectively given by the indices of the linearizations, $D^{P}$ and $D^{B}$, of the Cauchy Riemann operators $\bar{\partial}_{J_{P}}$ and $\bar{\partial}_{J_{B}}$, while the dimension of the moduli space in (2) is given by the index of $D^{v}$, the restriction of $D^{P}$ to vector fields along the curves that are vertically valued. The proof of Theorem B is based on the relation

$$
\pi_{*} \circ D^{P}=D^{B} \circ \pi_{*} \text {. }
$$

Consequently, $\pi$ induces a submersion of Fredholm systems (see Section 2) between the Fredholm systems relative to the operators $\bar{\partial}_{J_{P}}$ and $\bar{\partial}_{J_{B}}$, as 
defined in $[\mathbf{3}]$. From this we derive an exact sequence

$$
0 \rightarrow \operatorname{ker} D^{v} \rightarrow \operatorname{ker} D^{P} \rightarrow \operatorname{ker} D^{B} \rightarrow \operatorname{coker} D^{v} \rightarrow \text { coker } D^{P} \rightarrow \text { coker } D^{B} \rightarrow 0 .
$$

The result then follows by ensuring that the cokernels vanish, at least at the level of the universal moduli spaces, for example the hypothesis on $\sigma_{B}$ ensures that the last term of the sequence vanishes. It is worth pointing out that without this assumption standard transversality may fail a priori, due to multiple coverings as shown in [18]. The remaining obstructions are dealt with by perturbing the Hamiltonian connection as in [17], Chapter 8. More generally, $\bar{\pi}$ extends to a map, still denoted $\bar{\pi}$, between the compactifications $\overline{\mathcal{M}}_{0, l}\left(P, \sigma, J_{P}\right)$ and $\overline{\mathcal{M}}_{0, l}\left(B, \sigma_{B}, J_{B}\right)$ of the moduli spaces. These compactifications are stratified spaces for which the strata can be represented by stable stratum data $\mathcal{S}$, as pointed out by Kontsevitch, that is, by connected trees with tails together with an effective decomposition of the represented second homology class. We can repeat the arguments above for each stratum $\mathcal{M}_{\mathcal{S}_{P}}(P)$ mapped to a stratum $\mathcal{M}_{\mathcal{S}_{B}}(B)$ under $\bar{\pi}$, in order to show that transversality is generically realized for the irreducible elements in $\mathcal{M}_{\mathcal{S}_{P}}(P)$ whenever $\mathcal{M}_{\mathcal{S}_{B}}(B)$ does not contain reducible elements. Then condition $(\star \star)$ ensures that the "boundary" of the compactified moduli spaces above, given by lower strata, has codimension at least 2 with respect to the top stratum consisting of simple maps.

Once transversality is established, the product formula is obtained along the following lines. First, using the following commutative diagram of evaluation at the marked points maps:

$($ evd $)$

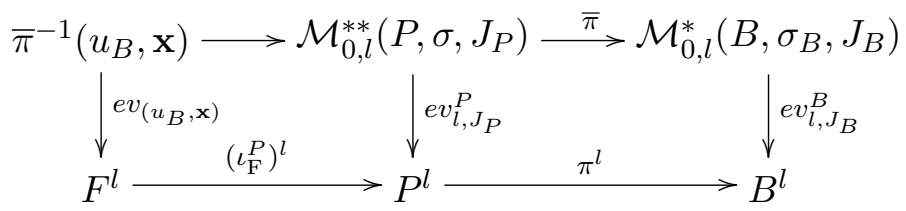

where

$$
e v_{\left(u_{B}, \mathbf{x}\right)}: \bar{\pi}^{-1}\left(u_{B}, \mathbf{x}\right) \rightarrow F^{l}, \quad(u, \mathbf{x}) \mapsto\left(u\left(x_{1}\right), \ldots, u\left(x_{l}\right)\right) \in \prod_{i=1}^{l} F_{u_{B}\left(x_{i}\right)},
$$

we consider the respective (transverse) intersections of these evaluations with product cycles, $\mathcal{C}^{B} \subset B^{l}, \mathcal{C}^{P} \subset P^{l}$ and $\mathcal{C}^{F} \subset F^{l}$, representing the products over $i=1, \ldots, l$ of the classes $c_{i}^{B}, c_{i}^{P}$ and $c_{i}^{F}$ in $(\star)$. The formula follows by observing that the intersection of $e v_{\left(u_{B}, \mathbf{x}\right)}$ with $\mathcal{C}^{F}$ does not depend on $\left(u_{B}, \mathbf{x}\right)$ counted in the intersection of $e v_{l, J_{B}}^{B}$ with $\mathcal{C}^{B}$, which is actually a consequence of the symplectic triviality of Hamiltonian fibrations over the 1-squeleton of their base $\left(H_{1}\right)$.

Remark 1.1. At this point, it is worth mentioning that the restriction to the genus 0 case is not essential. Although we have not treated the case of 
higher genus curves, all the results should still go through with minor modifications, except regarding the applications to $c$-splitting and symplectic uniruledness below. Another noteworthy observation is that the restrictions on $\sigma_{B}$ and the relative semipositivity conditions are only of technical order. It is believed that those ad hoc hypotheses can be avoided by using virtual perturbations (see $[\mathbf{3}, \mathbf{1 3}, \mathbf{2 0}, \mathbf{2 4}]$ ), which have been developped in order to deal with transversality issues for general symplectic manifolds. Removing these assumptions, is part of a joint work in progress with Shengda $\mathrm{Hu}$.

The second proof of the product formula is more global in essence: it rests on a better understanding, under some transversality assumptions, of the structure of the map $\bar{\pi}$. This is explained in more details in the next paragraph.

Fibration structure. It is natural to study the structure of the map

$$
\bar{\pi}: \overline{\mathcal{M}}_{0, l}\left(P, \sigma, J_{P}\right) \rightarrow \overline{\mathcal{M}}_{0, l}\left(B, \sigma_{B}, J_{B}\right) .
$$

In particular, it would be interesting to understand when $\bar{\pi}$ is a fibration, at least above the top stratum of the target space. When this is the case, we can recover the product formula using integration over the fibers of $\bar{\pi}$. Assuming the linearized operators involved in the exact sequence above are all surjective, it follows that the restriction of $\bar{\pi}$ to $\mathcal{M}_{0, l}\left(P, \sigma, J_{P}\right)$ is a smooth submersion onto $\mathcal{M}_{0, l}\left(B, \sigma_{B}, J_{B}\right)$. However, this map is not proper. This latter condition is important, as one can easily construct a smooth submersion that is not proper and which does not induce a fibration structure. To solve this problem, we consider the fiberwise compactification of $\bar{\pi}$. The properness issue then "disappears" but at the cost of losing the obvious smooth structure. Nevertheless, Chen and Li recently showed in the general case of a symplectic manifold $(X, \omega)$, that one can define a differentiable orbifold atlas on $\overline{\mathcal{M}}_{0, l}(X, A, J)$, where the charts are given by gluing maps [3]. There are many variants in the gluing of pseudo-holomorphic spheres procedure (see $[\mathbf{3}, \mathbf{1 4}, \mathbf{1 7}, \mathbf{2 0}, \mathbf{2 3}]$, among others), which appears naturally in the GW theory as well as in the Floer theory. The approach followed in $[\mathbf{3}]$ is to use balanced curves in order to define a natural slice for the action of the group $P S L_{2}(\mathbb{C})$ of reparameterizations of $S^{2}$, reducing the action of this latter non-compact group to that of $S^{1}$. As a consequence, they obtain gluing maps that are well-defined after quotient by the reparameterizations. Adapting their ideas to the Hamiltonian fibration case we construct gluing maps $G l^{P}$ and $G l^{B}$ satisfying

$$
\bar{\pi} \circ G l^{P}=G l^{B} \circ \bar{\pi} .
$$

Those maps are constructed under the assumption that $J_{P}$ is split regular (SR) with respect to $\sigma$ (see Section 6 , introduction). Roughly speaking, this means that on every stratum of $\overline{\mathcal{M}}_{0, l}\left(P, \sigma, J_{P}\right)$, mapped to a stratum of 
$\overline{\mathcal{M}}_{0, l}\left(B, \sigma_{B}, J_{B}\right)$ under $\bar{\pi}$, the operators in the exact sequence of the preceding paragraph are all surjective. Under the (SR) assumption we obtain the following result that can be seen as the global geometric incarnation of the product formula:

Theorem C. The moduli spaces $\overline{\mathcal{M}}_{0, l}\left(P, \sigma, J_{P}\right)$ and $\overline{\mathcal{M}}_{0, l}\left(B, \sigma_{B}, J_{B}\right)$ are $C^{1}$-orbifolds, and the map $\bar{\pi}$ restricts to a $C^{1}$ locally trivial fibration (of $C^{1}$-orbifolds) above each stratum of $\overline{\mathcal{M}}_{0, l}\left(B, \sigma_{B}, J_{B}\right)$. Moreover, the product formula can be recovered using integration over the fibers of $\bar{\pi}$ above the top stratum of $\overline{\mathcal{M}}_{0, l}\left(B, \sigma_{B}, J_{B}\right)$.

Proof. We only show how to recover the product formula assuming the first assertion of the theorem. The rest of the proof is postponed to Section 6 . In order to simplify notations, set $\mathcal{M}(P):=\mathcal{M}_{0, l}^{* *}\left(P, \sigma, J_{P}\right)$ and $\mathcal{M}(B):=$ $\mathcal{M}_{0, l}^{*}\left(B, \sigma_{B}, J_{B}\right)$. It actually follows from the split regularity assumption that the lower strata in $\overline{\mathcal{M}}(B)$, in $\overline{\mathcal{M}}(P)$, and in $\bar{\pi}^{-1}\left(u_{B}, \mathbf{x}\right)$ for every $\left(u_{B}, \mathbf{x}\right) \in$ $\mathcal{M}(B)$, are of codimension at least two (see Section 6, Introduction).

Now, let $c_{i}^{P}, c_{i}^{B}$ and $c_{i}^{F}, i=1, \ldots, l$, be classes as in $(\star)$. We can represent these classes by submanifolds after multiplying them by well chosen integers, if necessary. Represent the Poincaré duals of $c_{i}^{P}, c_{i}^{B}$ and $c_{i}^{F}$, by differential forms $\alpha_{i}^{P}, \alpha_{i}^{B}$ and $\alpha_{i}^{F}$, compactly supported in a small-enough tubular neighborhoods around the submanifolds. Without loss of generality we can assume that the evaluation maps $e v^{P}, e v^{B}$ and $e v_{\left(u_{B}, \mathbf{x}\right)}$ in (evd), are respectively transverse to the product cycles $\mathcal{C}^{P}, \mathcal{C}^{B}$ and $\mathcal{C}^{F}$. Since the inferior strata of the moduli spaces involved are of codimension at least two, the pull-backs of the $\alpha_{i}^{P}, \alpha_{i}^{B}$ and $\alpha_{i}^{F}$, along $e v^{P}, e v^{B}$ and $e v_{\left(u_{B}, \mathbf{x}\right)}$, are also compactly supported in $\mathcal{M}(P), \mathcal{M}(B)$ and in $\bar{\pi}^{-1}\left(u_{B}, \mathbf{x}\right)$. For $i=1, \ldots, m$, let $\rho_{i}: \mathcal{N}_{i} \rightarrow F$ denote a deformation retract associated to a tubular neighborhood $\mathcal{N}_{i}$ of the fiber above $c_{i}^{B}=p t$. From condition $(\star)$ we deduce that

$$
\begin{cases}\alpha_{i}^{B}=\operatorname{vol}(B), \quad \alpha_{i}^{P}=\pi^{*} \operatorname{vol}(B) \wedge \rho_{i}^{*} \alpha_{i}^{F}, & \text { for } i=1, \ldots, m, \\ \alpha_{i}^{F}=1_{F}, \quad \alpha_{i}^{P}=\pi^{*}\left(\alpha_{i}^{B}\right), & \text { for } i=m+1, \ldots, l,\end{cases}
$$

where $1_{F}$ stands for the constant function on $F$ equal to 1 . Here, we have to make sure that the support of $\pi^{*} \operatorname{vol}(B)$ does not strictly contain the support of $\rho_{i}^{*} \alpha_{i}^{F}$, but this can be realized by decreasing the support of $\operatorname{vol}(B)$ if necessary. By definition, (see [19]) we have that:

$$
\left\langle c_{1}^{P}, \ldots, c_{l}^{P}\right\rangle_{0, l, \sigma}^{P}=\int_{\mathcal{M}(P)}\left(e v^{P}\right)^{*}\left(\bigwedge_{i=1}^{l} \alpha_{i}^{P}\right)=\int_{\mathcal{M}(B)} \bar{\pi}_{*}\left(e v^{P}\right)^{*}\left(\bigwedge_{i=1}^{l} \alpha_{i}^{P}\right),
$$

where $\bar{\pi}_{*}$ stands for integration along the fibers. Using (1.3), we get

$$
\left.\left\langle c_{1}^{P}, \ldots, c_{l}^{P}\right\rangle_{0, l, \sigma}^{P}=\int_{\mathcal{M}(B)} \bar{\pi}_{*}\left(e v^{P}\right)^{*}\left(\bigwedge_{i=1}^{k}\left(\pi^{*} \alpha_{i}^{B} \wedge \rho_{i}^{*} \alpha_{i}^{F}\right)\right) \wedge \bigwedge_{i=k+1}^{l} \pi^{*} \alpha_{i}^{B}\right) .
$$


Let $e v_{i}^{P}$ be the projection of $e v^{P}$ on the $i$ th component of $P^{l}$ and define $e v_{i}^{B}$ and $e v_{\left(u_{B}, \mathbf{x}\right), i}$ similarly. For $i=1, \ldots, l, \bar{\pi} \circ e v_{i}^{P}=e v_{i}^{B} \circ \bar{\pi}$, so that

$$
\left\langle c_{1}^{P}, \ldots, c_{l}^{P}\right\rangle_{0, l, \sigma}^{P}=(-1)^{\alpha} \int_{\mathcal{M}(B)} \bar{\pi}_{*}\left(\bigwedge_{i=1}^{l} \pi^{*}\left(e v_{i}^{B}\right)^{*} \alpha_{i}^{B} \wedge \bigwedge_{i=1}^{k}\left(e v_{i}^{P}\right)^{*} \rho_{i}^{*} \alpha_{i}^{F}\right),
$$

where $\alpha=\sum_{i=k+1}^{l} \operatorname{deg} \alpha_{i}^{B} \sum_{i=1}^{k} \operatorname{deg} \alpha_{i}^{F}$ is odd (by a simple dimension argument). Furthermore, since $\pi_{*}\left(a \wedge \pi^{*} b\right)=\left(\pi_{*} a\right) \wedge b$ for any form $a$ and $b$, we finally have:

$$
\left\langle c_{1}^{P}, \ldots, c_{l}^{P}\right\rangle_{0, l, \sigma}^{P}=\int_{\mathcal{M}(B)}\left(\left(e v^{B}\right)^{*} \bigwedge_{i=1}^{l} \alpha_{i}^{B}\right) \wedge \bar{\pi}_{*}\left(\bigwedge_{i=1}^{k}\left(e v_{i}^{P}\right)^{*} \rho_{i}^{*} \alpha_{i}^{F}\right),
$$

where the term involving integration over the fibers of $\pi$ must be a function $\psi$ on $\mathcal{M}(B)$ given by:

$$
\begin{aligned}
\psi\left(u_{B}, \mathbf{x}\right) & =\int_{\bar{\pi}^{-1}\left(u_{B}, \mathbf{x}\right)} e v_{\left(u_{B}, \mathbf{x}\right)}^{*}\left(\bigwedge_{i=1}^{k} \alpha_{i}^{F}\right)=\int_{\bar{\pi}^{-1}\left(u_{B}, \mathbf{x}\right)} e v_{\left(u_{B}, \mathbf{x}\right)}^{*}\left(\bigwedge_{i=1}^{l} \alpha_{i}^{F}\right) \\
& =\sum_{\sigma^{\prime} \in B_{\sigma}}\left\langle\iota_{\mathrm{F}}^{P_{C}}\left(c_{1}^{F}\right), \ldots, \iota_{\mathrm{F}}^{P_{C}}\left(c_{l}^{F}\right)\right\rangle_{0, l, \sigma^{\prime}}^{P_{C}},
\end{aligned}
$$

where $C$ denotes the image of $u_{B}$. By lemma $(2.3), \psi$ does not depend on $\left(u_{B}, \mathbf{x}\right)$ and we can withdraw it from the integral. This ends the proof.

An example. We illustrate the results above by giving an example of computation using the product formula. We also describe the corresponding induced fibration of moduli spaces and show that it is non-trivial.

Let $\pi: P \rightarrow \mathbb{C P}^{2}$ be the Hamiltonian fibration with fiber $\left(F:=\mathbb{C P}^{1}, \omega_{F S}\right)$ where $P$ is the projectivization of the rank 2 holomorphic vector bundle

$$
\pi: V:=\mathcal{O}_{\mathbb{C P}^{2}}(1) \oplus \mathbb{C} \rightarrow \mathbb{C P}^{2} .
$$

Let $J_{0}$ be the standard complex structure on $\mathbb{C P}^{2}$ that is compatible with $\omega_{F S}$. Let $J_{P}$ be the integrable structure on $P$ induced by $J_{0}$, the structure of complex fibration on $V$ and the holomorphic Hermitian connection on $V$ (inducing the coupling form here.).

Let $h \in H^{2}(P, \mathbb{Z})$ denote the pull-back under $\pi$ of the positive generator in $H^{2}\left(\mathbb{C P}^{2}, \mathbb{Z}\right)$ Poincaré dual to the class $L \in H_{2}\left(\mathbb{C P}^{2}, \mathbb{Z}\right)$ of a line in $\mathbb{C P}^{2}$. Let also $\xi \in H^{2}(P ; \mathbb{Z})$ be the first Chern class of the dual of the tautological bundle over $P$. The following description for the cohomology ring $H^{*}(P ; \mathbb{Z})$ is standard (see [1] , Chapter IV):

$$
H^{*}(P ; \mathbb{Z}) \cong \frac{\mathbb{Z}[h, \xi]}{\left\{h^{3}=0, \xi^{2}+h \xi=0\right\}} .
$$


Then, $H_{2}(P ; \mathbb{Z})$ is generated by the duals of the classes $h^{2}$ and $h \xi$. Let $L_{0} \in$ $H_{2}(P, \mathbb{Z})$ denote the Poincaré dual of $h^{2}+h \xi$. If $\pi_{*}$ represents integration along the fibers of $\pi$ then $\pi_{*} L_{0}=L$.

The map $\pi$ induces the projection

$$
\bar{\pi}: \overline{\mathcal{M}}\left(P, L_{0}, J_{P}\right) \rightarrow \overline{\mathcal{M}}\left(\mathbb{C P}^{2}, L, J_{0}\right) \cong\left(\mathbb{C P}^{2}\right)^{*}
$$

The source moduli space is made of two strata: $\mathcal{S}_{0}$ the top stratum of simple maps, and $\mathcal{S}_{1}$ the stratum consisting of stable maps having two components, one being a $\pi_{*}$-stable component (cf Section 4.1.1) representing the class of the exceptional divisor, $P D(h \xi)$, and the other being a $\pi_{*}$-unstable component (cf Section 4.1.1) representing the class $P D\left(h^{2}\right)=[F]$. Observe that the second stratum contains only irreducible elements.

Lemma 1.1. The fibered complex structure $J_{P}$ is $S R$ for $L_{0}$.

Proof. First, since $L$ is $J_{0}$-indecomposable, coker $D^{B}$ vanishes in the exact sequence of linearized operators. Thus, we have to verify that coker $D^{v}=0$ on every stratum. But, for every $u \in \mathcal{S}_{0}$, we have

$$
\text { coker } \begin{aligned}
D_{u}^{v} & =H^{0,1}\left(\mathbb{C P}^{1}, u^{*} T P^{v}\right)=H^{0,1}\left(\mathbb{C P}^{1}, \mathcal{O}_{\mathbb{C P}^{2}}(1)\right) \\
& \cong H^{0}\left(\mathbb{C P}^{1}, \mathcal{O}(-3)\right)=0 .
\end{aligned}
$$

Now consider $\left(u_{1}, u_{2}, y_{12}, y_{21}\right) \in \mathcal{S}_{1}$, where $u_{1}$ denotes the $\pi_{*}$-stable component, and $u_{2}$ denotes the $\pi_{*}$-unstable component. Again, we have to check that coker $D_{u_{1}}^{v}$ and coker $D_{u_{2}}^{v}$ vanish. Moreover, we have to show that for every line $\ell \in\left(\mathbb{C P}^{2}\right)^{*}$ the edge evaluation map

$e v: \bar{\pi}^{-1}(\ell) \cap \mathcal{S}_{1} \rightarrow \pi^{-1}(b) \times \pi^{-1}(b), \quad\left(u_{1}, u_{2}, y_{12}, y_{21}\right) \mapsto\left(u_{1}\left(y_{12}\right), u_{1}\left(y_{21}\right)\right)$, is transverse to the diagonal $\Delta_{\pi^{-1}(b)}$, where $b:=\pi(\bar{b})$ and $\bar{b}$ denotes the unique intersection point between $u_{1}$ and $u_{2}$. Again, for every holomorphic map representing $[F]$, coker $D_{u_{2}}^{v}$ can be identified to $H^{0,1}\left(\mathbb{C P}^{1}, T \mathbb{C P}^{1}\right)$, which vanishes, and similarly

$$
\text { coker } D_{u_{1}}^{v}=H^{0,1}\left(\mathbb{C P}^{1}, u_{1}^{*} T P^{v}\right)=H^{0,1}\left(\mathbb{C P}^{1}, \mathcal{O}(-1)\right)=0 .
$$

Finally, transversality of $e v$ follows since for every $X_{0} \in T_{\bar{b}} \pi^{-1}(b)$ there exists a holomorphic vector field on $\mathbb{C P}^{1} \cong \pi^{-1}(b)$ with value $X_{0}$ at $\bar{b}$.

As an example, we compute $\langle p t, p t\rangle_{0,2, L_{0}}^{P}$. Since the homology of the fiber injects in the homology of the total space, the product formula simplifies to give:

$$
\langle p t, p t\rangle_{0,2, L_{0}}^{P}=\langle p t, p t\rangle_{0,2, L}^{\mathbb{C P}^{2}} \cdot\langle p t, p t\rangle_{0,2, B}^{\left.P\right|_{\ell}},
$$

where $\ell$ is the line in $\mathbb{C P}^{2}$ passing through two given points and $B$ is the Poincaré dual of the restriction of $h^{2}+h \xi$ to $\left.P\right|_{\ell}$. Note that the restriction of $P$ to a line in $\mathbb{C P}^{2}$ is the projectivization of $\mathcal{O}_{\mathbb{C P}^{1}}(1) \oplus \mathbb{C}$, hence is isomorphic to $\widetilde{\mathbb{C P}}^{2}$, the one point blow-up of $\mathbb{C P}^{2}$. Then, the class $B$ corresponds to the 
sum of the fiber and the exceptional divisor in the blow up. It follows that both members of the above product give 1 , hence

$$
\langle p t, p t\rangle_{0,2, L_{0}}^{P}=1 \text {. }
$$

Next, we show that $\bar{\pi}$ is non-trivial, more precisely that it is a $\mathbb{C P}^{2}$ fibration obtained as the projectivization of a non-trivial rank two holomorphic bundle over $\left(\mathbb{C P}^{2}\right)^{*}$. The fibers of $\bar{\pi}$ are given by $\overline{\mathcal{M}}\left(\widetilde{\mathbb{C P}}^{2}, L_{0}, J\right)$, where $J$ is the complex structure associated to the Hirzebruch surface $\mathbb{P}\left(\mathcal{O}_{\mathbb{C P}^{1}}(1) \oplus \mathbb{C}\right)$, and $L_{0}$ is the class represented by the zero section. Furthermore,

$$
\mathcal{M}^{*}\left(\widetilde{\mathbb{C P}}^{2}, L_{0}, J\right) \cong H^{0}\left(\mathbb{C P}^{1}, \mathcal{O}_{\mathbb{C P}^{1}}(1)\right) \cong \mathbb{C}\langle u, v\rangle
$$

where $[u: v]$ stands for the homogeneous coordinates on $\mathbb{C P}^{1}$. The Gromov closure then corresponds to adding the line at infinity, so that we have $\overline{\mathcal{M}}\left(\widetilde{\mathbb{C P}}^{2}, L_{0}, J\right)=\mathbb{C P}^{2}$. Now, consider the incidence variety:

$$
W:=\left\{(p, \ell) \in \mathbb{C} P^{2} \times\left(\mathbb{C P}^{2}\right)^{*} \mid p \in \ell\right\},
$$

and let $\pi_{1}, \pi_{2}$ denote the projections on the first and second factors. Note that $\left(W, \pi_{2}\right)$ is the projectivization of $\mathcal{O}_{\left(\mathbb{C P}^{2}\right)^{*}}(-1) \oplus \mathbb{C}$. Consider the direct image sheaf over $\left(\mathbb{C P}^{2}\right)^{*}$,

$$
\mathcal{R}:=\pi_{2 *} \pi_{1}^{*} \mathcal{O}_{\mathbb{C P}^{2}}(1)
$$

whose germ at $\ell \in\left(\mathbb{C P}^{2}\right)^{*}$ is given by

$$
H^{0}\left(\pi_{2}^{-1}(\ell),\left.\mathcal{O}_{\mathbb{C P}^{1}}(1)\right|_{\pi_{2}^{-1}(\ell)}\right) \cong H^{0}\left(\mathbb{C P}^{1}, \mathcal{O}_{\mathbb{C P}^{1}}(1)\right) .
$$

Hence $\mathcal{R}=\mathcal{S}_{0}$. Let $\mathcal{D}$ be a line in $\left(\mathbb{C P}^{2}\right)^{*}$. Then $\pi_{2}^{-1}(\mathcal{D})$ can be identified to $\widetilde{\mathbb{C P}}^{2}$, where the blown-up point is given by the intersection of all the lines generated by $\mathcal{D}$. In fact,

$$
\pi_{2}^{-1}(\mathcal{D}) \cong \frac{D^{+} \times \mathbb{C P}^{1} \sqcup D^{-} \times \mathbb{C P}^{1}}{(\lambda,[u: v]) \sim\left(\lambda^{-1},[\lambda u: v]\right), \lambda \neq 0},
$$

where $D^{+}$and $D^{-}$respectively denote the complements of $[0: 1]$ and $[1: 0]$ in $\mathbb{C P}^{1}$. Hence, the restriction of $\mathcal{R}$ to $\mathcal{D}$ is the direct sum of $\mathcal{O}_{\mathbb{C P}^{1}}(-1)$ with $\mathbb{C}$, so that $\bar{\pi}$ is non-trivial.

Applications. In 1997, Seidel defined in [22] a representation of the space of Hamiltonian loops of a given symplectic manifold in the automorphism group of the corresponding quantum homology. Lalonde, McDuff and Polterovich have shown, under the relative semi-positivity assumption, that the rational cohomology of the total space splits as a module for any Hamiltonian fibration over $S^{2}[\mathbf{1 1}]$. McDuff removed the semi-positivity assumption using virtual techniques [15]. In general, we say that a fibration is 
rationally c-split if

$$
H^{*}(P ; \mathbb{Q}) \cong H^{*}(B ; \mathbb{Q}) \otimes H^{*}(F ; \mathbb{Q})
$$

as modules. This splitting is realized when $\iota_{\mathrm{F}}^{P}: F \hookrightarrow P$ induces an injection in rational homology, and if, in addition, the second page of the Leray-Serre spectral sequence splits. More generally, Lalonde and McDuff conjectured that every Hamiltonian fibration verifies the $c$-splitting property [10]. They showed that this splitting property holds for a large panel of Hamiltonian fibrations, in particular for Hamiltonian fibrations over $\mathbb{C P}^{n}$. Below we provide an alternative proof that Hamiltonian fibrations over $\mathbb{C P}^{n}$ with relative semi-positive fiber are $c$-split, using the product formula and the invertibility of Seidel's morphism $[\mathbf{1 1}, \mathbf{2 2}]$.

Another consequence is the symplectic uniruledness of Hamiltonian fibrations over rationally connected bases. As defined in [6], a symplectic manifold $(X, \omega)$ is (symplectically) uniruled if there is a non-vanishing $G W$ invariant with at least one point as a constraint. In other words, if there exists $A \in H_{2}(X, \mathbb{Z})$ and homology classes $c_{2}, \ldots, c_{l} \in H_{*}(X)$ such that:

$$
\left\langle p t, c_{2}, c_{3}, \ldots, c_{l}\right\rangle_{0, l, A}^{X} \neq 0 .
$$

A symplectic manifold is rationally connected if there is a non-zero $G W$ invariant involving two point insertions [6], i.e., the equation above is still true with $c_{2}=p t$. In summary:

Corollary. Let $\pi: P \rightarrow B$ be a Hamiltonian fibration. Assume $B$ is rationally connected with respect to a class $\sigma_{B} \in H_{2}(B ; \mathbb{Z})$ verifying the hypothesis of Theorem B. Then, $P$ is c-split and symplectically uniruled.

Proof. Let $C$ be the image of a map counted in $\left\langle p t, p t, c_{3}^{B}, \ldots, c_{l}^{B}\right\rangle_{0, l, \sigma_{B}}^{B} \neq 0$. As already mentioned, $P_{C}$ is a Hamiltonian fibration over $S^{2}$, and by a result of Lalonde, McDuff and Polterovich, [11] (Corollary 4.C.), for every $a \in H_{*}(F)$ there is an equivalence class $\sigma^{\prime}$ of section classes in $P_{C}$, as well as an element $b \in H_{*}(F)$, such that:

$$
0 \neq\left\langle\iota_{\mathrm{F}}^{P_{C}}(a), \iota_{\mathrm{F}}^{P_{C}}(b)\right\rangle_{0,2, \sigma^{\prime}}^{P_{C}}=\left\langle\iota_{\mathrm{F}}^{P_{C}}(a), \iota_{\mathrm{F}}^{P_{C}}(b), \iota_{\mathrm{F}}^{P_{C}}([F]), \ldots, \iota_{\mathrm{F}}^{P_{C}}([F])\right\rangle_{0, l, \sigma^{\prime}}^{P_{C}},
$$

where the last equality is a consequence of the Divisor axiom $([\mathbf{1 7}]$, Section 7.5.). Applying the product formula, as given in (1.2), we conclude that:

$$
\left\langle\iota_{\mathrm{F}}^{P}(a), \iota_{\mathrm{F}}^{P}(b), \pi^{-1}\left(c_{3}^{B}\right), \ldots, \pi^{-1}\left(c_{l}^{B}\right)\right\rangle_{0, l, \iota_{P_{C}}^{P}(\sigma)}^{P} \neq 0 .
$$

Hence, by taking $a=p t$ we obtain that $P$ is uniruled. Now, suppose $\pi$ is not c-split. Then there exists $a \in H_{*}(F ; \mathbb{Z})$ in the kernel of $\iota_{\mathrm{F}}^{P}$. Therefore, the $G W$-invariants having $\iota_{\mathrm{F}}^{P}(a)$ as an entry, must vanish. But this would contradict (1.4). 
The proof of this corollary indicates that unless we have a good knowledge of Seidel's morphism the number of point insertions should a priori decrease. Still, as a result, every Hamiltonian fibration over $\left(\mathbb{C P}^{n}, \omega_{F S}\right)$ is $c$-split and uniruled, where $\omega_{F S}$ is the standard Kähler form on the complex projective space. The same applies for Hamiltonian fibrations over $\left(S^{2} \times S^{2}, \omega \oplus \omega\right)$ since in that case there is only one curve representing the diagonal and passing through two points.

The paper is organized as follows. Section 1, introduces the basic ingredients needed. We also define a particular affine connection on $P$, whose torsion is given by the symplectic curvature associated to the coupling form. In Section 2, we describe the linearization of the Cauchy-Riemann problem associated to the fibered almost complex structures. It is shown that the linearization is compatible with the projection $\pi$. Then we prove the structure theorems in Section 4, ensuring the $G W$-invariants are well defined. In Section 5 , we give the proof of the product formula. Section 6 , is devoted to showing the locally trivial differentiable (orbi-)fibration structure of $\bar{\pi}$.

\section{The framework}

In this section, we set the basic notions that will be needed in the rest of the paper. We recall that symplectic and Hamiltonian fibrations are classified by $\operatorname{BSymp}\left(F, \omega_{\mathrm{F}}\right)$ and $\operatorname{BHam}\left(F, \omega_{\mathrm{F}}\right)$, respectively. Again, for $b \in B$, let $\omega_{b}$ be the induced symplectic form on the fiber $F_{b}:=\pi^{-1}(b)$. For the sake of clarity, we begin by recalling the notions of Hamiltonian connections and coupling form. This exposition follows [5] (Chapter 1) and [16] (Chapter 6), where the proofs of all the claims can be found.

2.1. Hamiltonian connections and coupling form. Consider the vertical subbundle, Vert $\subset T P$, over $P$, whose fiber at each point, $p \in P$, is given by the subspace $\operatorname{Vert}_{p}:=\operatorname{ker} d \pi(p)$. A connection on the fibration, $\pi: P \rightarrow B$, is defined by a splitting of $T_{p} P$ for each $p \in P$ :

$$
T_{p} P=\operatorname{Hor}(p) \oplus \operatorname{Vert}_{p},
$$

where $\operatorname{Hor}(p)$ is called the horizontal plane at $p$. The notations, $X^{h}$ and $X^{v}$, will refer to the horizontal and vertical parts of a vector field $X$ on $P$ with respect to the above splitting. Also, given a vector field $X$ on $B$, we will denote by $\bar{X}$ its horizontal lift to $T P$. Now, let $R$ denote the the symplectic curvature associated to the connection. This is the 2 -form on $B$ with values in Vert such that, for $v$ and $w$ two vector fields on $B$,

$$
R(v, w)(p):=[\bar{v}, \bar{w}]^{v}(p), \quad p \in P .
$$

Any closed extension 2-form $\tau$ of $\omega_{\mathrm{F}}$ defines a connection with horizontal planes:

$$
\operatorname{Hor}_{\tau}(p):=\left\{v \in T_{p} P \mid \tau(v, w)=0 \forall w \in \operatorname{Vert}_{p}\right\} .
$$


In fact, any form $\tau^{\prime} \in \Omega^{2}(P)$ such that $\operatorname{ker}\left(\tau^{\prime}-\tau\right)$ is in Vert defines the same horizontal plane field. Nevertheless, a specific choice can be made by requiring that the integration of $\tau^{n+1}$ over the fibers of $P$ is zero. Such $\tau$ is called coupling form associated to the connection and its values on pairs of horizontal vectors is determined by the curvature of the connection:

$$
-d(\tau(p)(v, w)):=\iota(R(d \pi(p) v, d \pi(p) w)(p)) \omega_{\pi(p)}(p) .
$$

This results from the fact that the holonomy of the connection is Hamiltonian.

For transversality purposes we will need to allow the connection to vary. For $b \in B$, let $C_{0}^{\infty}\left(F_{b}\right)$ denote the space of smooth functions on $F_{b}$ having zero mean value. We will consider Hamiltonian deformations of the coupling form $\tau$. By this we mean exact deformations,

$$
\tau_{H}:=\tau-d \tilde{H}
$$

where $\widetilde{H}$ is a section in $C^{\infty}\left(\pi^{*} T^{*} B\right)$, i.e., $\widetilde{H}_{p}$ is a cotangent vector in $T_{\pi(p)} B$ and it satisfies the property that for fixed $b \in B$ and $X \in T_{b} B$ the function $H_{b, X}(p):=\widetilde{H}_{p}(X)$ belongs to $C_{0}^{\infty}\left(F_{b}\right)$. The subset of $C^{\infty}\left(\pi^{*} T^{*} B\right)$ having this property will be denoted by $\mathcal{H}$. By definition, $\tau_{H}$ is a closed extension 2 -form of $\omega_{\mathrm{F}}$, and one verifies that its associated horizontal distribution is given by:

$$
\operatorname{Hor}_{\tau_{H}}(p)=\left\{v-X_{\widetilde{H}_{p}(v)}(p) \mid v \in \operatorname{Hor}_{\tau}(p)\right\}, \quad p \in P,
$$

where $X_{\widetilde{H}_{p}(v)}$ denotes the (unique) Hamiltonian vector field on $F_{\pi(p)}$ induced by the function $\widetilde{H}_{p}(v)$.

Remark 2.1. Since we are working with functions having zero mean value, $\tau_{H}$ is nothing else but the coupling form associated to Hor $\tau_{\tau_{H}}$. Also, observe that the symplectic curvature changes under exact deformation.

2.2. Almost complex structures. An almost complex structure $J$ on a symplectic manifold, $(F, \omega)$, is a smooth section of the bundle of endomorphisms of the tangent space of $F$ such that:

$$
\forall p \in F, \quad(J(p))^{2}=-i d_{T_{p} F} .
$$

Let $\mathcal{J}(F)$ denote the set of almost complex structures on $F$ and let $\mathcal{J}_{\mathrm{F}} \equiv \mathcal{J}(F, \omega)$ be the subset of $\mathcal{J}(F)$ consisting of $\omega$-tame almost complex structures, i.e., such that $\omega(\cdot, J \cdot)$ is positive definite. A choice of such a $J$ gives $T F$ the structure of a complex fiber bundle. Let $c_{1}^{T F} \in H^{2}(F, \mathbb{Z})$ denote the corresponding first Chern class. Since $\mathcal{J}_{\mathrm{F}}$ is contractible and nonempty ([16], Chapter 2), this class is well defined and does not depend on our choice of compatible almost complex structure. Let $\pi: P \rightarrow\left(B, \omega_{B}\right)$ be a Hamiltonian fibration with fiber $(F, \omega)$ and coupling form $\tau$. 
Definition 2.1. An almost complex structure $J_{P} \in \mathcal{J}(P)$ is compatible with $\pi$ and $\tau$, or fibered, if and only if there exists $J_{B} \in \mathcal{J}\left(B, \omega_{B}\right)$ such that:

- $d \pi \circ J_{P}=J_{B} \circ d \pi$,

- $J_{b}:=\left.J_{P}\right|_{F_{b}} \in \mathcal{J}\left(F_{b}, \omega_{b}\right)$ for all $b \in B$,

- $J_{P}$ preserves the horizontal distribution induced by $\tau$.

We denote by $\mathcal{J}(P, \tau, \pi)$ the set of such almost complex structures.

Each fibered almost complex structure determines a family $\left\{J_{b}\right\}_{b \in B}$ of $\omega_{b}$-tame almost complex structures. Let $\mathcal{J}^{V} \equiv \mathcal{J}^{V}(P, \pi, \omega)$ denote the set of almost complex structures of the vertical subbundle, which are $\omega_{b}$-tame on each fiber $F_{b}$. Also, let $c^{v} \in H^{2}(P, \mathbb{Z})$ be the corresponding first Chern class. Conversely, for any $J \in \mathcal{J}^{V}$ and $J_{B} \in \mathcal{J}_{B}$, there exists a unique fibered structure $J_{P}$ extending $J$ and defined as the horizontal lift of $J_{B}$ on the horizontal distribution. Furthermore, when deforming the coupling form using $H \in \mathcal{H}$, the unique extension of $J$ that projects on $J_{B}$ and that preserves the distribution induced by $\tau_{H}$ is given by:

$$
J_{P}^{H}(p)(v)=J_{P}(p)(v)+J(p) X_{H_{\pi(p)}\left(\pi_{* p} v\right)}-X_{H_{\pi(p)}\left(J_{B}(\pi(p)) \pi_{* p} v\right)}, \quad p \in P,
$$

where $v \in T_{p} P$. Let $\mathcal{J}_{P} \equiv \mathcal{J}(P, \pi)$ denote the union of the $\mathcal{J}\left(P, \pi, \tau_{H}\right)$ over all deformations $\tau_{H}$ of a given coupling form $\tau$. We have:

Lemma 2.1. The space $\mathcal{J}_{P}$ is parameterized by the product $\mathcal{J}_{B} \times \mathcal{J}^{V} \times \mathcal{H}$.

The above isomorphism is given by the choice of $\tau$. In fact, the dependence is on the factor $\mathcal{H}$ corresponding to affine space of Hamiltonian connections. The choice of $\tau$ simply fixes the origin.

Remark 2.2. Note that, for any family $\left\{J_{b}\right\}_{b \in B} \in \mathcal{J}^{V}$ and any given $J_{B}$, we can find a positive $\kappa \in \mathbb{R}$ such that $J_{P}$ is $\omega_{P}$-tame for $\omega_{P}=\tau+\kappa \pi^{*} \omega_{B}$.

2.3. A specific affine connection. Fix a coupling form $\tau$. We define an affine connection on TP extending the vertical Levi-Civita $(L-C)$ connection introduced in $[\mathbf{1 7}]$ (Chapter 8) and lifting the $\mathrm{L}-\mathrm{C}$ connection on $T B$. This construction will be needed in order to relate the linearization of the CauchyRiemann associated to $J_{P}=\left(J_{B}, J, H\right) \in \mathcal{J}_{P}$, to the linearization of the Cauchy-Riemann operator associated to $J_{B}$ (see Section 2). First, let $g_{J_{P}}$ be the Hermitian metric on $P$ defined as

$$
g_{J_{P}}:=g_{J} \oplus \pi^{*} g_{J_{B}},
$$

relatively to the splitting $T P=\operatorname{Vert} \oplus \operatorname{Hor}_{\tau_{H}}$, where

$$
g_{J_{B}}:=\frac{1}{2}\left(\omega_{B}\left(., J_{B} .\right)-\omega_{B}\left(J_{B} ., .\right)\right),
$$

and $g_{J}:=\left\{g_{J_{b}}\right\}_{b \in B}$ is the analogous family of Hermitian metrics on Vert. Let $\nabla^{B}$ denote the $\mathrm{L}-\mathrm{C}$ connection on $T B$ relatively to $g_{J_{B}}$. Also, set $\nabla^{F}$ to 
be the $\mathrm{L}-\mathrm{C}$ connection on $T F_{b}$, with $b \in B$, relatively to $g_{J_{b}}$. For any vector fields $X$ and $Y$ on $P$, set

$$
\nabla_{X} Y:=\left[X^{h}, Y^{v}\right]^{v}+\nabla_{X_{v}}^{F} Y^{v}+\left[X^{v}, Y^{h}\right]^{h}+\overline{\left(\nabla_{\pi_{*} X^{h}}^{B} \pi_{*} Y^{h}\right)}
$$

This operation is clearly bilinear in $X$ and $Y$. In fact, the sum of the first two terms corresponds to the vertical $L-C$ connection, $\nabla^{v}$, which is the unique connection on Vert induced by the Hamiltonian connection and which restricts to the $\mathrm{L}-\mathrm{C}$ connection on $F$. The remaining part is what is needed to extend this vertical connection to an affine connection on $P$ lifting the $\mathrm{L}-\mathrm{C}$ connection on $B$, which torsion $T$ is given by the symplectic curvature:

$$
T(X, Y)=-R(X, Y)=\left[X^{h}, Y^{h}\right]^{v}, \quad X, Y \in \mathcal{X}(T P) .
$$

We show below that $\nabla$ is indeed a connection. Since $\nabla^{v}$ is a connection, it suffices to show that for $f \in C^{\infty}(P)$ and $\xi \in \operatorname{Hor}_{\tau}$, and any $w \in T P$ :

$$
\nabla_{w} f \xi=(w(f))(\xi)+f \nabla_{w} \xi, \quad \nabla_{f w} \xi=f \nabla_{w} \xi
$$

Suppose that $w$ is vertical. Then, by definition:

$$
\nabla_{w} f \xi=[w, f \xi]^{h}=f[w, \xi]^{h}+w(f) \xi .
$$

Analogously, we have $[f w, \xi]=f[w, \xi]-\xi(f) w$, implying that

$$
[f w, \xi]^{h}=f[w, \xi]^{h}
$$

since $w$ is vertical. Now, suppose that $w$ is horizontal. Let $\alpha_{t}, t \in(-\epsilon, \epsilon)$ be the flow of $w$ starting at $p$, and let $P_{t}^{B}\left(\pi_{*_{p}} w_{p}\right)$ denote the parallel transport along the projected curve $\pi\left(\alpha_{t}\right)(p)$. Then,

$$
\begin{aligned}
\left(\nabla_{w} f \xi\right)_{p} & =\left.\frac{d}{d t}\right|_{t=0}\left(f\left(\alpha_{t}(p)\right) P_{t}^{B}\left(\pi_{*_{p}} w_{p}\right) \pi_{*_{\alpha_{t}(p)}} \xi\right)_{p}^{h} \\
& =d f(p) w \xi+f(p)\left(\nabla_{w} \xi\right)_{p},
\end{aligned}
$$

where the first equality is given by linearity of the parallel transport in $B$. We further have that

$$
\nabla_{f w} \xi=\left.\frac{d}{d t}\right|_{t=0}\left(P_{t}^{B}\left(\pi_{*_{p}} f(p) w_{p}\right) \pi_{*_{\alpha_{t}}(p)} \xi\right)_{p}^{h}=\left.\frac{d}{d t}\right|_{t=0}\left(P_{t}^{B}\left(\pi_{*_{p}} w_{p}\right) \pi_{*_{\alpha_{\psi}(t)}(p)} \xi\right)_{p}^{h}
$$

for some reparameterization $\psi(t)$ of the interval, so that finally $f \nabla_{w} \xi$.

Remark 2.3. Note that $\nabla^{v}$ may not preserve the metric $g_{J}$ along horizontal directions, whereas $\widetilde{\nabla}^{v}=\nabla^{v}-\frac{1}{2} J\left(\nabla^{v} J\right)$ does ([17], Lemma 8.3.6). Furthermore, given any vector fields $w, \xi_{1}$ and $\xi_{2}$ in $T P$, one can show that $\left(\nabla_{w} g\right)\left(\xi_{1}, \xi_{2}\right)$ coincides with $\left(\nabla_{w^{h}} g\right)\left(\xi_{1}^{v}, \xi_{2}^{v}\right)$. It follows that the $J_{P}$-preserving connection $\widetilde{\nabla}:=\nabla-\frac{1}{2} J_{P}\left(\nabla J_{P}\right)$ preserves $g_{J_{P}}$. 
Let exp stand for both the exponential maps with respect to $\nabla$ and $\nabla^{B}$. The following straightforward identities will be useful in the gluing section:

Lemma 2.2. For $p \in P, X \in T_{p} P$ and $q \in P$ in the injective radius of $\exp _{p}$, we have

$$
\pi\left(\exp _{p} X\right)=\exp _{\pi(p)} \pi_{*_{p}} X, \quad \pi_{*_{p}} \exp _{p}^{-1}(q)=\exp _{\pi(p)}^{-1}(\pi(q)) .
$$

2.4. Curve independence. From $\left(H_{1}\right)$ in the characterization of Hamiltonian fibrations we deduce the following lemma, which plays a crucial part in the proof of the product formula:

Lemma 2.3. Let $\pi: P \rightarrow B$ be a Hamiltonian fibration as above. Assume we have $u_{1}, u_{2} \in C^{\infty}\left(S^{2}, B\right)$ such that $\left[u_{1}\left(S^{2}\right)\right]=\left[u_{2}\left(S^{2}\right)\right]$. Then the restricted bundles $\left.P\right|_{u_{1}}$ and $\left.P\right|_{u_{2}}$ are isomorphic as Hamiltonian bundles.

Proof. If $B$ is simply connected then it follows directly from Hurewicz isomorphism between $\pi_{2}(B)$ and $H_{2}(B, \mathbb{Z})$. Assume $B$ is not simply-connected. As $\operatorname{Ham}(F, \omega)$ is connected, any classifying map for $P$, say $f$, factorizes up to homotopy through a map $f^{\prime}: B / B_{1} \rightarrow \operatorname{BHam}(F, \omega)$. In other words, if $\pi_{B_{1}}$ denotes the projection from $B$ to $B / B_{1}$, the maps $f$ and $f^{\prime} \circ \pi_{B_{1}}$ are homotopic. Let $P^{\prime}:=\left(f^{\prime}\right)^{*} E \operatorname{Ham}(F, \omega)$ and consider

$$
u_{1}^{\prime}:=\pi_{B_{1}} \circ u_{1}, \quad u_{2}^{\prime}:=\pi_{B_{1}} \circ u_{2} .
$$

These two maps represent the same homology class $\pi_{B_{1 *}}\left(\sigma_{B}\right) \in H_{2}\left(B / B_{1} ; \mathbb{Z}\right)$, so that $u_{1}^{\prime}$ and $u_{2}^{\prime}$ are homotopic and:

$$
\left.\left.P\right|_{u_{1}} \cong\left(u_{1}^{\prime}\right)^{*} P^{\prime} \cong\left(u_{2}^{\prime}\right)^{*} P^{\prime} \cong P\right|_{u_{2}} .
$$

\section{The Cauchy-Riemann problem in Hamiltonian fibrations}

Let $j_{0}$ denote the complex structure on $S^{2}=\mathbb{C} \cup\{\infty\}$ inherited from the multiplication by $i:=\sqrt{-1}$. In a general symplectic manifold $(X, \omega)$ with $\omega$-tame almost complex structure $J$, a rational $J$-holomorphic map is a smooth map, $u: S^{2} \rightarrow X$, satisfying the Cauchy-Riemann equation,

$$
\bar{\partial}_{J} u:=\frac{1}{2}\left(d u+J \circ d u \circ j_{0}\right)=0 .
$$

The set of all such solutions representing a given class $A \in H_{2}(X, \mathbb{Z})$,

$$
\widetilde{\mathcal{M}}(X, A, J):=\left\{u \in C^{\infty}\left(S^{2}, X\right) \mid \bar{\partial}_{J} u=0,\left[u\left(S^{2}\right)\right]=A\right\},
$$

is the moduli space of parameterized J-holomorphic maps representing A. Consider the Fréchet space,

$$
\mathcal{B}_{X}(A):=\left\{u \in C^{\infty}\left(S^{2}, X\right) \mid\left[u\left(S^{2}\right)\right]=A\right\},
$$


and the space

$$
\mathcal{E}_{X}(A, J)=\bigsqcup_{u \in \mathcal{B}_{X}(A)} \mathcal{E}_{X, u}(J):=\bigsqcup_{u \in \mathcal{B}_{X}(A)} C^{\infty}\left(\Lambda_{J}^{0,1}\left(S^{2}, u^{*} T X\right)\right) .
$$

The obvious projection from $\mathcal{E}_{X}(A, J)$ to $\mathcal{B}_{X}(A)$ defines a locally trivial bundle between Fréchet spaces. Then, $\bar{\partial}_{J}$ is a section of this bundle and $\widetilde{\mathcal{M}}(X, A, J)$ is the corresponding zero set. The linearized operator $D_{u}^{X}$ of $\bar{\partial}_{J}$ at $u \in \widetilde{\mathcal{M}}(X, A, J)$ is defined as the differential of $\bar{\partial}_{J}$ at $u$ composed with the projection on the fiber $\mathcal{E}_{X, u}(J)$. To give a meaning to this vertical projection outside of the zero section, we consider the Hermitian connection on $\mathcal{E}_{X}(A, J)$,

$$
\widetilde{\nabla}^{X}:=\nabla^{X}-1 / 2\left(J\left(\nabla^{X} J\right)\right),
$$

where $\nabla^{X}$ is the $\mathrm{L}-\mathrm{C}$ connection on $X$ with respect to the metric $g_{J}$. Set $\mathcal{X}_{X, u}:=C^{\infty}\left(S^{2}, u^{*} T X\right)$. Then,

$$
\begin{aligned}
D_{u}^{X}: \mathcal{X}_{X, u} & \rightarrow \mathcal{E}_{X, u}(J), \\
\xi & \mapsto \widetilde{\nabla}_{\xi}^{X} \bar{\partial}_{J}(u)=\left(\nabla_{d u}^{X}\right)^{0,1} \xi-\frac{1}{2} J(u)\left(\nabla_{\xi}^{X} J\right)\left(\partial_{J} u\right),
\end{aligned}
$$

where $\left(\nabla^{X}\right)^{0,1}$ is the $J$-anti-linear part of $\nabla^{X}$. It is well known that $D_{u}^{X}$ is Fredholm for $u \in \widetilde{\mathcal{M}}(X, A, J)$. Moreover, if $\bar{\partial}_{J}$ is transversal to the zero section, then the moduli space is a smooth finite-dimensional oriented manifold with dimension given by the index of $D^{X}$ (cf [17], Theorem 3.1.5):

$$
\operatorname{dim} \widetilde{\mathcal{M}}(X, A, J)=\operatorname{Ind}\left(D^{X}\right)=2 n_{X}+2 c_{1}^{T X}(A) .
$$

Now, let $\pi: P \rightarrow B$ be a Hamiltonian fibration with coupling form $\tau$, let $J_{P}$ be a fibered complex structure relatively to $\tau$, and let $\sigma \in H_{2}(P, \mathbb{Z})$. The connection $\nabla$ we constructed induces a splitting of the tangent space of $\mathcal{E}_{P}\left(\sigma, J_{P}\right)$ at all points and projection to the fiber direction can again be defined. Hence, the linearization $D_{u}^{P}$ of $\bar{\partial}_{J_{P}}$ can be defined for all $u \in \mathcal{B}_{P}(\sigma)$. Note that $\nabla$ is not Levi-Civita since its torsion is given by the symplectic curvature. This is what gives rise to the extra curvature term in the following expression for $D_{u}^{P}$ :

Lemma 3.1. Let $u \in \mathcal{B}_{P}(\sigma)$ and $\xi \in C^{\infty}\left(S^{2}, u^{*} T P\right)$. Then,

$$
D_{u}^{P} \xi=\left(\nabla_{d u}\right)^{0,1} \xi-\frac{1}{2} J_{P}(u)\left(\nabla_{\xi} J_{P}\right)\left(\partial_{J_{P}} u\right)+R^{0,1}\left(d u^{h}, \xi^{h}\right),
$$

where $\nabla^{0,1}$ and $R^{0,1}$ stand for the $J_{P}$ anti-linear parts of $\nabla$ and $R$.

Remark 3.1. When $\xi$ is vertically valued, the curvature term disappears and we recover the vertical linearized operator introduced by McDuff and Salamon $([\mathbf{1 7}]$, Chapter 8$)$. In the rest of the paper we will designate by $D^{v}$ the restriction of $D^{P}$ to vertically valued vector fields. 
3.1. Splitting of Fredholm systems. Since we consider fibered almost complex structures on $P$, the projection $\pi$ naturally induces a map,

$$
\pi: \widetilde{\mathcal{M}}\left(P, \sigma, J_{P}\right) \rightarrow \widetilde{\mathcal{M}}\left(B, \sigma_{B}, J_{B}\right), \quad u \mapsto \pi(u),
$$

where $\sigma_{B}:=\pi_{*} \sigma$. As we will see, $\pi$ induces a submersion between the Fredholm system $\left(\mathcal{B}_{P}(\sigma), \mathcal{E}_{P}\left(\sigma, J_{P}\right), \bar{\partial}_{J_{P}}\right)$ and $\left(\mathcal{B}_{B}\left(\sigma_{B}\right), \mathcal{E}_{B}\left(\sigma_{B}, J_{B}\right), \bar{\partial}_{J_{B}}\right)$. We recall the notion of Fredholm system as given in [3], Section 14.2.

Definition 3.1. A Fredholm system of index $d$ is a triple $(\mathcal{B}, \mathcal{E}, s)$ such that:

$\left(F_{1}\right) \mathcal{E}$ is the total space of a Banach orbifold bundle over a Banach orbifold $\mathcal{B}$, with fiber $\mathcal{E}_{x}$ over $x \in \mathcal{B}$,

$\left(F_{2}\right) s: \mathcal{B} \longrightarrow \mathcal{E}$ is a section such that, for all $x \in s^{-1}(0)$, the linearization $L_{x}: T_{x} \mathcal{B} \rightarrow \mathcal{E}_{x}$ of $s$ at $x$, is Fredholm of index $d$.

When, in addition, $s$ is proper, we say that the system is compact.

We call the set, $s^{-1}(0)$, the moduli space of the system. The use of orbifolds in the definition above is due to the presence of non-simple maps in the concrete problem of Cauchy-Riemann Fredholm system. This will be relevant in Section 6 .

Example 3.1. Let $(X, \omega)$ be a general symplectic manifold with $A \in$ $H_{2}(X, \mathbb{Z})$ and $\omega$-tame almost complex structure $J$. Fix an integer $p>2$. For $u \in \mathcal{B}_{X}(A)$, we respectively equip $\mathcal{X}_{X, u}$ and $\mathcal{E}_{X, u}(J)$ with $W^{1, p}$ (Sobolev) and $L^{p}$ norms (relatively to the metric $g_{J}$ and a fixed volume form on $S^{2}$ ). Explicitly, given $\xi \in \mathcal{X}_{X, u}$ and $\eta \in \mathcal{E}_{X, u}(J)$ :

$$
\|\xi\|_{W^{1, p}}=\left(\int_{S^{2}}\left(|\xi|_{g_{J}}^{p}+|\nabla \xi|_{g_{J}}^{p}\right) d \operatorname{vol}_{S^{2}}\right)^{\frac{1}{p}}, \quad\|\eta\|_{L^{p}}=\left(\int_{S^{2}}|\xi|_{g_{J}}^{p} d \operatorname{vol}_{S^{2}}\right)^{\frac{1}{p}} .
$$

We denote by $\mathcal{X}_{X, u}^{1, p}$ and $\mathcal{E}_{X, u}^{p}(J)$ the completed vector spaces with respect to these norms, and by $\mathcal{B}_{X}^{1, p}(A)$ and $\mathcal{E}_{X}^{p}(A, J)$ the corresponding completions of $\mathcal{B}_{X}(A)$ and $\mathcal{E}_{X}(A, J)$. Under these completions $D_{u}^{X}$ is Fredholm, and the triple

$$
\left(\mathcal{B}_{X}^{1, p}(A), \mathcal{E}_{X}^{p}(A, J), \bar{\partial}_{J}\right)
$$

satisfies conditions $\left(F_{1}\right)$ and $\left(F_{2}\right)$. This Fredholm system will in general not be compact.

Next, we define the following natural notion of morphism between two Fredholm systems $(\mathcal{B}, \mathcal{E}, s)$ and $\left(\mathcal{B}^{\prime}, \mathcal{E}^{\prime}, s^{\prime}\right)$.

Definition 3.2. A map $\Pi:=(\pi, \bar{\pi}):(\mathcal{B}, \mathcal{E}, s) \rightarrow\left(\mathcal{B}^{\prime}, \mathcal{E}^{\prime}, s^{\prime}\right)$ between Fredholm systems is a Banach orbifold vector bundle morphism, i.e.,

$$
\pi: \mathcal{B} \rightarrow \mathcal{B}^{\prime}, \quad \bar{\pi}: \mathcal{E} \rightarrow \mathcal{E}^{\prime},
$$

such that 
(i) $s^{\prime} \circ \pi=\bar{\pi} \circ s$,

(ii) $L_{\pi(x)}^{\prime} \circ d \pi(x)=d \bar{\pi}(x, 0) \circ L_{x}$, for every $x \in s^{-1}(0)$.

We say that $\Pi$ is a submersion if, furthermore, $d \pi$ and $d \bar{\pi}$ are surjective.

When $\Pi$ is a submersion we directly extract the following exact sequence: (3.1) $\operatorname{ker} L_{x}^{\prime \prime} \longmapsto \operatorname{ker} L_{x} \rightarrow \operatorname{ker} L_{\pi(x)}^{\prime} \rightarrow \operatorname{coker} L_{x}^{\prime \prime} \rightarrow \operatorname{coker} L_{x} \rightarrow \operatorname{coker} L_{\pi(x)}^{\prime}$. where $x \in s^{-1}(0)$ and $L_{x}^{\prime \prime}: \operatorname{ker} d \pi \rightarrow \operatorname{ker} d \bar{\pi}$ is the restriction of $L_{x}$ to ker $d \pi(x)$. This exact sequence is an instance of the cohomology long exact sequence of 2-step complexes determined by $L^{\prime \prime}, L$ and $L^{\prime}$. From exactness of this sequence we deduce splitting at the level of operator indices, i.e., for every $x \in s^{-1}(0)$ :

$$
\operatorname{Ind}\left(L_{x}\right)=\operatorname{Ind}\left(L_{\pi(x)}^{\prime}\right)+\operatorname{Ind}\left(L_{x}^{\prime \prime}\right) .
$$

Obviously, if both $L_{x}^{\prime \prime}$ and $L_{\pi(x)}^{\prime}$ are unobstructed (i.e., their cokernels vanish), then $L_{x}$ is also unobstructed. As a result, $\operatorname{ker} L_{x}$ is isomorphic to $\operatorname{ker} L_{x}^{\prime \prime} \oplus \operatorname{ker} L_{\pi(x)}^{\prime}$ up to a choice of a section from $\operatorname{ker} L_{\pi(x)}^{\prime}$ to $\operatorname{ker} L_{x}$.

Definition 3.3. A submersion $\Pi$ between Fredholm systems is a splitting if the sequence (3.1) is obstruction free, i.e., if for all $x \in s^{-1}(0)$ :

$$
\text { coker } L_{x}^{\prime \prime}=\text { coker } L_{x}=\text { coker } L_{\pi(x)}^{\prime}=0 .
$$

3.2. Splitting of Fredholm systems for Hamiltonian fibrations. Here, we show that a Hamiltonian fibration $\pi: P \rightarrow B$ with coupling form $\tau$ induces a submersion between the Fredholm systems $\left(\mathcal{B}_{P}(\sigma), \mathcal{E}_{P}\left(\sigma, J_{P}\right), \bar{\partial}_{J_{P}}\right)$ and $\left(\mathcal{B}_{B}\left(\sigma_{B}\right), \mathcal{E}_{B}\left(\sigma_{B}, J_{B}\right), \bar{\partial}_{J_{B}}\right)$. In fact, $\pi$ induces the maps:

$$
\pi: \mathcal{B}_{P}(\sigma) \rightarrow \mathcal{B}_{B}\left(\sigma_{B}\right), \quad f \mapsto \pi \circ f
$$

and

$$
\bar{\pi}: \mathcal{E}_{P}\left(\sigma, J_{P}\right) \longrightarrow \mathcal{E}_{B}\left(\sigma_{B}, J_{B}\right), \quad \eta \mapsto \pi_{*}(\eta) .
$$

That $\pi$ induces a submersion between Fredholm systems can be summarized as follows:

Lemma 3.2. The following diagrams are commutative:

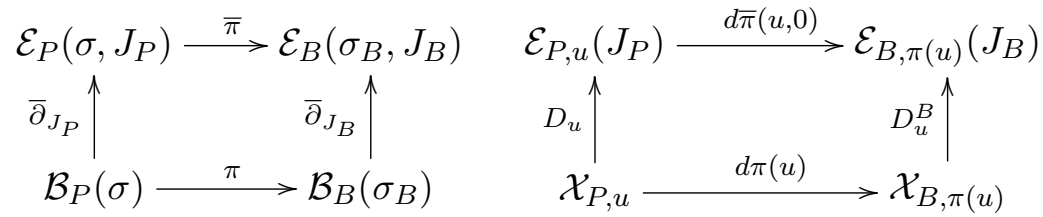

Proof. For the left hand-side diagram, commutativity follows from the fact that $J_{P}$ is fibered. Thus, $\pi$ maps $\left(\bar{\partial}_{J_{P}}\right)^{-1}(0)$ into $\left(\bar{\partial}_{J_{B}}\right)^{-1}(0)$. We prove that the second diagram commutes. First, note that $d \pi$ and $d \bar{\pi}$ are surjective 
since we can use the Hamiltonian connection to lift vector fields on $B$ to vector fields on $P$. Next, we show that for every $\xi \in \mathcal{X}\left(u^{*} T P\right)$ :

$$
\pi_{*} D_{u} \xi=D_{\pi \circ u}^{B} \pi_{*} \xi
$$

This is a consequence of the following three identities: let $\xi$ and $X$ be vector fields on $P$ and let $p \in P$, then

(i) $\pi_{*}\left[\xi^{v}, J_{P} X^{h}\right]=J_{B} \pi_{*}\left[\xi^{v}, X^{h}\right]$,

(ii) $\pi_{*}\left(\nabla_{\xi} J_{P}\right)_{p} X=\left(\nabla_{\pi_{*} \xi}^{B} J_{B}\right)_{\pi(p)}\left(\pi_{*} X\right)$,

(iii) $\pi_{*}\left(\nabla_{d u}^{0,1} \xi\right)=\left(\nabla_{\pi_{*} d u}^{B}\right)^{0,1} \pi_{*} \xi-\pi_{*}\left[\left(\bar{\partial}_{J_{P}}(u)\right)^{h}, \xi^{v}\right]^{h}$.

Assuming these are verified we obtain that

$\pi_{*} D_{u} \xi=\left(\nabla_{d(\pi \circ u)}^{B}\right)^{0,1} \pi_{*} \xi-\pi_{*}\left[\left(\bar{\partial}_{J_{P}}(u)\right)^{h}, \xi^{v}\right]^{h}-\frac{1}{2} J_{B}(\pi \circ u)\left(\nabla_{\pi_{*} \xi}^{B} J_{B}\right)\left(\pi_{*} \partial_{J_{P}} u\right)$,

since $\nabla_{d u}^{0,1} \xi^{v}$ and $R^{0,1}(d u, \xi)$ are vertically valued. Moreover, since $\pi_{*} \partial_{J_{P}} u=$ $\partial_{J_{B}} u_{B}$ it only remains to show that:

$$
\left[\left(\bar{\partial}_{J_{P}}(u)\right)^{h}, \xi^{v}\right]_{u(z)}^{h}=0 \quad \forall z \in S^{2} .
$$

If $\left(\bar{\partial}_{J_{P}} u\right)(z)^{h} \neq 0$ for some $z$, consider the horizontal lift $\bar{X}$ of $\left(\bar{\partial}_{J_{B}}(\pi(u))\right)(z)$, which is defined on $T\left(\pi^{-1}(u(z))\right)$ and agrees with $\left(\bar{\partial}_{J_{P}} u\right)(z)^{h}$ at $u(z)$. Then,

$$
\left[\left(\bar{\partial}_{J_{P}}(u)\right)^{h}, \xi^{v}\right]_{u(z)}=\left[\bar{X}, \xi^{v}\right]_{u(z)}
$$

where the right hand-side vanishes since $\bar{X}$ is constant along vertical directions.

Now, equality (i) follows by definition of the bracket, by holomorphicity of the projection, and since the flow of a vertical vector field starting at a point $p$ remains in the fiber above $\pi(p)$. Equality (ii) is just a consequence of (i) since this latter is equivalent to

$$
\left[\xi^{v}, J_{P} X^{h}\right]^{h}=J_{P}\left[\xi^{v}, X^{h}\right]^{h},
$$

which by definition of $\nabla$ is the same as:

$$
\nabla_{\xi^{v}}\left(J_{P} X^{h}\right)=J_{P} \nabla_{\xi^{v}}\left(X^{h}\right)
$$

Hence, $\left(\nabla_{\xi^{v}} J_{P}\right)\left(X^{h}\right)=0$, which combined with the fact that the connection is vertically valued when its two entries are in Vert, gives us the second equation.

For (iii) we have that:

$$
\nabla_{d u}^{0,1} \xi=\frac{1}{2}\left(\nabla_{\xi} d u+J_{P}(u) \nabla_{\xi}\left(d u \circ j_{0}\right)\right)-R^{0,1}(d u, \xi) .
$$


Hence, by definition of the connection and because the curvature is vertically valued, we get

$$
\begin{aligned}
2 \pi_{*}\left(\nabla_{d u}^{0,1} \xi\right)= & \pi_{*} \nabla_{\xi} d u+J_{B}(\pi(u)) \pi_{*} \nabla_{\xi}\left(d u \circ j_{0}\right) \\
= & \nabla_{\pi_{*} \xi}^{B} d(\pi \circ u)+\pi_{*}\left[\xi^{v},(d u)^{h}\right]^{h}+J_{B}(\pi(u)) \nabla_{\pi_{*} \xi}^{B} d(\pi \circ u) \circ j_{0} \\
& +J_{B}(\pi(u)) \pi_{*}\left[\xi^{v},\left(d u \circ j_{0}\right)^{h}\right]^{h} \\
= & \nabla_{d(\pi \circ u)}^{B} \pi_{*} \xi+J_{B}(\pi(u)) \nabla_{d(\pi \circ u) \circ j_{0}}^{B} \pi_{*} \xi \\
& +\pi_{*}\left[\xi^{v},(d u)^{h}+J_{P}(u)\left(d u \circ j_{0}\right)^{h}\right]^{h} \\
= & 2\left(\nabla_{d(\pi \circ u)}^{B}\right)^{0,1} \pi_{*} \xi+\pi_{*}\left[\xi^{v}, 2\left(\bar{\partial}_{J_{P}}(u)\right)^{h}\right]^{h}
\end{aligned}
$$

where the third equality is due to (i) and the fact that $\nabla^{B}$ is torsion free. The last follows since $J_{P}$ preserves the horizontal distribution and the vertical subbundle.

The symplectic connection on $P$ induces the splittings:

$$
\mathcal{E}_{P, u}=\Gamma\left(\Lambda_{J_{P}}^{0,1}\left(S^{2}, u^{*} T P^{h}\right)\right) \oplus \Gamma\left(\Lambda_{J_{P}}^{0,1}\left(S^{2}, u^{*} T P^{v}\right)\right)=: \mathcal{E}_{P, u}^{h}\left(J_{P}\right) \oplus \mathcal{E}_{P, u}^{v}\left(J_{P}\right)
$$

and

$$
\mathcal{X}_{P, u}=\Gamma\left(S^{2}, u^{*} T P^{h}\right) \oplus \Gamma\left(S^{2}, u^{*} T P^{v}\right)=\mathcal{X}_{P, u}^{h} \oplus \mathcal{X}_{P, u}^{v} .
$$

In this splitting, $D_{u}^{P}$ takes the following matrix form:

$$
\left(\begin{array}{cc}
\left(D_{\pi(u)}^{B}\right)^{h} & 0 \\
L_{u} & D_{u}^{v}
\end{array}\right)
$$

where $L_{u}$ is linear and given by:

$$
L_{u}: \mathcal{X}_{P, u}^{h} \longrightarrow \mathcal{E}_{P, u}^{v}, \quad \xi \mapsto-\frac{1}{2} J(u)\left(\nabla_{\xi} J\right)\left(\partial_{J_{P}} u\right)^{v}+R^{0,1}\left(d u^{h}, \xi\right) .
$$

Thus, applying the diagram (3.1), we obtain the exact sequence:

$$
\operatorname{ker} D_{u}^{v} \longmapsto \operatorname{ker} D_{u}^{P} \rightarrow \operatorname{ker} D_{\pi(u)}^{B} \rightarrow \operatorname{coker} D_{u}^{v} \rightarrow \operatorname{coker} D_{u}^{P} \rightarrow \operatorname{coker} D_{\pi(u)}^{B}
$$

where the connectant is given by the restriction of $L_{u}$ to the kernel of $D_{\pi(u)}^{B}$.

In the next section we will show that, for generic fibered almost complex structure, the last two terms of this exact sequence vanish. We also show that, generically, the induced projection between the moduli spaces is a submersion along countably many fibers of this projection, i.e., the fourth term vanishes on countably many fibers. In fact, this will be proved by establishing that the projection of the Hamiltonian fibration induces a splitting of Fredholm system when we allow the fibered almost complex structure to vary as well. 


\section{Structure theorems}

Let $\widetilde{\mathcal{M}}_{0, l}\left(P, \sigma, J_{P}\right)$ be the moduli space of parameterized $J_{P}$-holomorphic maps with $l$ marked points representing $\sigma$. This space consists of tuples,

$$
\left(u, x_{1}, \ldots, x_{l}\right) \in \widetilde{\mathcal{M}}\left(P, \sigma, J_{P}\right) \times\left(S^{2}\right)^{l},
$$

where the points $x_{1}, \ldots, x_{l} \in S^{2}$ are pairwise distinct. The group $G:=$ $P S L_{2}(\mathbb{C})$ of reparameterizations of $S^{2}$ acts (diagonally) on this moduli space. The quotient of $\widetilde{\mathcal{M}}_{0, l}\left(P, \sigma, J_{P}\right)$ under this action is usually not compact. Still, its "Gromov's compactification", $\overline{\mathcal{M}}_{0, l}\left(P, \sigma, J_{P}\right)$, is a stratified space consisting of stable holomorphic maps $[\mathbf{7}, \mathbf{1 7}, \mathbf{2 1}]$. Concretely, the stratification is given by the combinatorial-type of the labeled connected trees with tails modeling the stable maps. When these strata are automorphism free they can be given a manifold structure for a generic choice of fibered almost complex structure. After giving the description of the stable holomorphic maps in Hamiltonian fibrations, we show that the latter structure theorem holds compatibly with the projection $\pi$. This is the content of Theorem B' which is a slight extension of Theorem B. We conclude by observing that changing of generic almost complex structure induces a cobordism between the corresponding moduli spaces.

4.1. Stable holomorphic maps and Hamiltonian fibrations. In order to fix notations and terminology we begin by introducing, first, the combinatorics needed to describe stable holomorphic maps, and second, the moduli space of stable pseudo-holomorphic maps.

4.1.1. Labeled graphs. This exposition is mostly taken from [4]. Nevertheless, emphasis is put on how the combinatorics change when given a homomorphism of semi-groups. This will be particularly relevant when describing how the combinatorial-type of a pseudo-holomorphic stable map in $P$ changes when projected to $B$ under $\pi$.

Let $\mathcal{S}$ be a graph with tails; let $V$ denote its set of vertices, let $E$ denote its set of edges, and let $T$ denote its set of tails. In order to indicate that there is an edge between the $v$ and the $w$ vertices of $\mathcal{S}$, we will use the notation $v E w$. The genus $g(\mathcal{S})$ of the graph is the Euler number of $\mathcal{S}$, i.e.,

$$
g(\mathcal{S})=|V|-|E|+1 .
$$

If all the connected components of $\mathcal{S}$ have genus 0 we say that it is a forest; if, furthermore, $\mathcal{S}$ is connected we say that it is a tree. In the rest of the paper we will only consider forests. We will use the notation $C$ to denote the set of connected components of $\mathcal{S}$.

To describe the combinatorics of the graph it is convenient to see the tails as half-edges and the edges as two half-edges that are attached together. Let Fl denote the set of half-edges of $\mathcal{S}$. This set comes with a natural map, 
pr $: \mathrm{Fl} \rightarrow V$, assigning to a half-edge the vertex it is attached to. Hence, $\mathrm{Fl}_{v}:=\operatorname{pr}^{-1}(v)$ gives the set of valences at $v \in V$. We set

$$
|v|:=\#\left(\operatorname{pr}^{-1}(v)\right) \text {. }
$$

The incidence relations between the vertices of $\mathcal{S}$ are encoded using an involution $\varrho: \mathrm{Fl} \rightarrow \mathrm{Fl}$. More precisely, $E$ corresponds to the 2-elements orbits of $\varrho$ while the fixed points of $\varrho$ give $T$. The graph $\mathcal{S}$ is determined uniquely by the tuple $(V, \mathrm{Fl} ; \mathrm{pr}, \varrho)$ and we write $\mathcal{S}=(V, \mathrm{Fl} ; \mathrm{pr}, \varrho)$. The subgraph $\left(\{v\}, \mathrm{Fl}_{v} ;\left.\mathrm{pr}\right|_{\mathrm{Fl}_{v}}, i d\right)$ is called the star of $\mathcal{S}$ at $v$. Note that $\mathcal{S}$ can be obtained from its stars by gluing them according to the involution $\rho$; see Figure 1 below: in that picture $\varrho$ fixes $a_{i}$ and $b_{i}, i=1,2$, and sends $c_{1}$ to $c_{2}$.

Given an Abelian semi-group $B$, a $B$-labeling of $\mathcal{S}$ is simply a map $\beta$ : $V \rightarrow B$. We denote by $(\mathcal{S}, \beta)$ the corresponding labeled graph. A $B$-labeling $\beta$ induces a $B$-labeling of $C$ :

$$
\beta_{C}: C \rightarrow B, \quad \beta_{C}(c):=\sum_{v \in c} \beta(v) .
$$

For example, when each vertex of the graph corresponds to a Riemann surface, as is the case for the stable maps, we can consider the genus-labeling $h: V \rightarrow \mathbb{N}$ assigning to each vertex the genus of the corresponding Riemann surface. Since we are only dealing with genus 0 stable maps, the genuslabeling will always be trivial.

Next, we define a partial order on graphs. For this purpose we introduce the following notion of composition of graphs: the composition $\mathcal{S}^{\prime \prime}:=\left(\mathcal{S}^{\prime} \Rightarrow\right.$ $\mathcal{S}$ ) of the graph $\mathcal{S}^{\prime}$ with the graph $\mathcal{S}$ is the graph obtained by replacing the vertices in $\mathcal{S}$ by the connected components $C^{\prime}$ of $\mathcal{S}^{\prime}$. Concretely, one replaces each star at a vertex of $\mathcal{S}$ by a connected component of $\mathcal{S}^{\prime}$; this is illustrated in Figure 1. Composition is realizable if and only if there are set isomorphisms $\phi: T^{\prime} \rightarrow \mathrm{Fl}$ and $\psi: C^{\prime} \rightarrow V$ such that $\psi \circ \operatorname{pr}_{C^{\prime}}=\operatorname{pr} \circ \phi$, where $\operatorname{pr}_{C^{\prime}}: T^{\prime} \rightarrow C^{\prime}$ is the map assigning to a tail the connected component it is attached to. If such isomorphisms exist, we say that $\mathcal{S}^{\prime}$ and $\mathcal{S}$ are composable. It is not hard to see that $\mathcal{S}^{\prime \prime}$ is given by $\left(V^{\prime}, \mathrm{Fl}^{\prime} ; \mathrm{pr}^{\prime}, \varrho^{\prime \prime}\right)$ where $\varrho^{\prime \prime}$ coincides with $\varrho^{\prime}$ on edges of $\mathcal{S}^{\prime}$ and coincides with $\varrho$ otherwise (using the identity $\phi \circ \varrho^{\prime \prime}=\varrho \circ \phi$ on tails of $\mathcal{S}^{\prime}$ ).

Example 4.1. In Figure 1, the isomorphisms $\phi$ and $\psi$ are given as follows: $\psi\left(v_{i}^{\prime}\right)=v_{i}, \phi\left(a_{i}^{\prime}\right)=a_{i}, \phi\left(b_{i}^{\prime}\right)=b_{i}$ and $\phi\left(c_{i}^{\prime}\right)=c_{i}$, where $i=1,2$.

Labeled graphs are also composable: let $(\mathcal{S}, \beta)$ and $\left(\mathcal{S}^{\prime}, \beta^{\prime}\right)$ be labeled graphs such that $\mathcal{S}^{\prime}$ is composable with $\mathcal{S}$, and let $\pi: B^{\prime} \rightarrow B$ be a homomorphism of semi-groups. The graphs are $\pi$-composable if $\beta=\pi \circ \beta_{C^{\prime}}$.

There are two natural possibilities of labeling for the resulting graph $\mathcal{S}^{\prime \prime}$, namely $\beta^{\prime \prime}:=\pi \circ \beta^{\prime}$ or $\beta^{\prime}$. The $\pi$-composition is the $B$-labeled graph $\left(\mathcal{S}^{\prime \prime}, \beta^{\prime \prime}\right)$, while the labeled composition is the $B^{\prime}$-labeled graph $\left(\mathcal{S}^{\prime \prime}, \beta^{\prime}\right)$. 


\section{CLÉMENT HYVRIER}

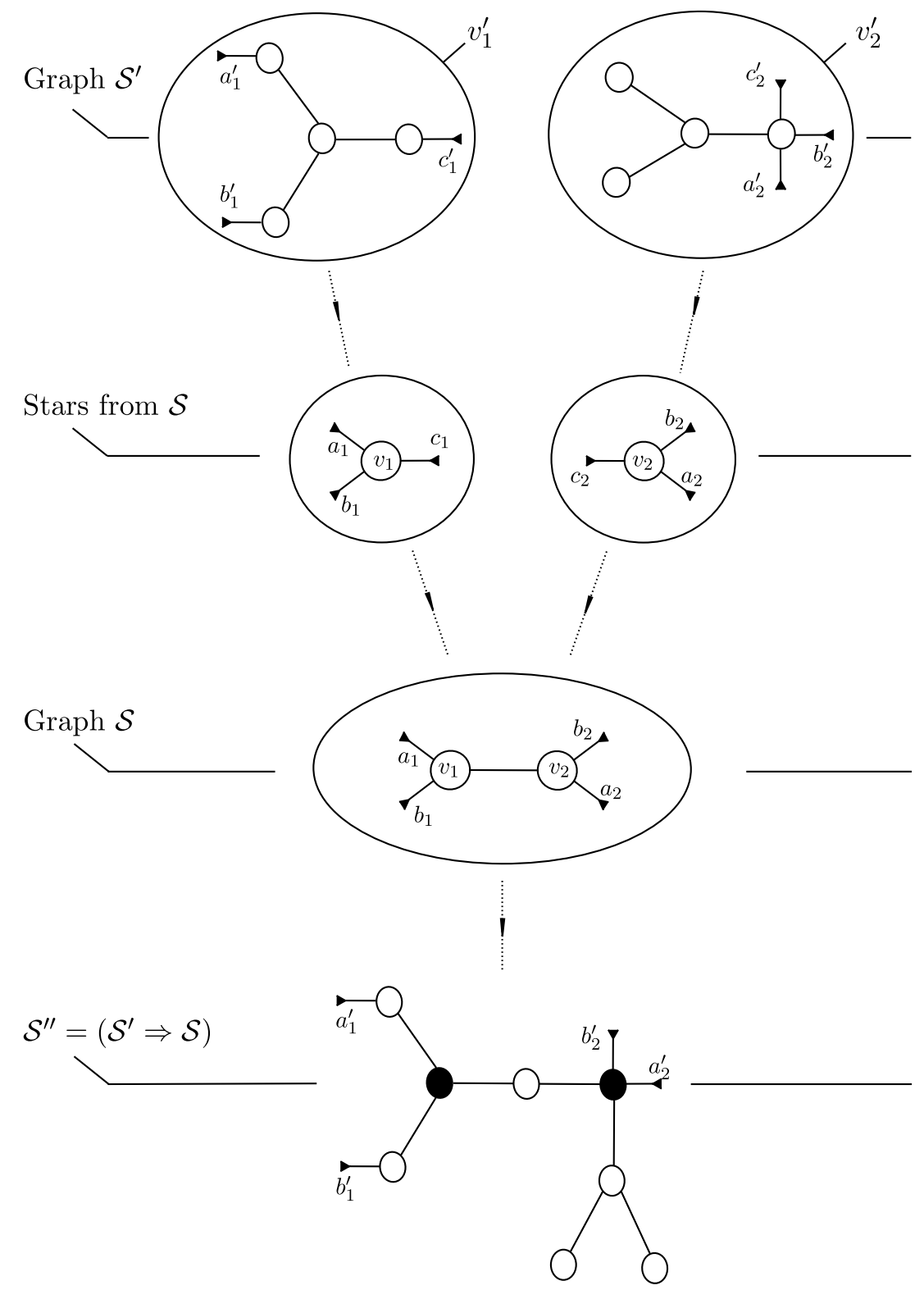

Figure 1. Composition of graphs.

The partial ordering $\prec$ is now defined as follows: $\mathcal{S}^{\prime \prime} \prec \mathcal{S}$ if and only if there exists a graph $\mathcal{S}^{\prime}$ such that $\mathcal{S}^{\prime \prime}=\left(\mathcal{S}^{\prime} \Rightarrow \mathcal{S}\right)$. This inequality can also be understood in terms of contractions: $\mathcal{S}$ is obtained from $\mathcal{S}^{\prime \prime}$ by contracting 
some subgraph of $\mathcal{S}^{\prime \prime}$. The components of this subgraph are called contracted components. This contraction procedure is not well-defined. For example, the graph with one vertex and no half-edge can be obtained in two different ways from the graph with two vertices, $v_{1}$ and $v_{2}$ say, and no half-edge: we can either contract $v_{1}$ or $v_{2}$. In fact, the contraction procedure is determined up to a choice of section of the composition of graphs. We explain this in more details.

The composition of graphs induces surjective maps $\gamma: V^{\prime \prime} \rightarrow V$ and $\gamma_{\mathrm{Fl}}: \mathrm{Fl}^{\prime \prime} \rightarrow \mathrm{Fl}$ via the isomorphisms $\phi: T^{\prime} \rightarrow \mathrm{Fl}$ and $\psi: C^{\prime} \rightarrow V$. A section of the composition is a pair of right inverses, $\iota: V \rightarrow V^{\prime \prime}$ and $\iota_{\mathrm{Fl}}: \mathrm{Fl} \rightarrow \mathrm{Fl}^{\prime \prime}$, such that $\iota \circ \mathrm{pr}=\mathrm{pr}^{\prime \prime} \circ \iota_{\mathrm{Fl}}$. In other words the section sends a star of $\mathcal{S}$ to a star of $\mathcal{S}^{\prime \prime}$. If the graphs are labeled, we further require that $\beta^{\prime \prime} \circ \iota=\pi \circ \beta^{\prime} \circ \iota=\beta$. In that case, we say that $(\iota, \iota \mathrm{Fl})$ is a labeled section. Note that a section always exists while a labeled one may not.

Now, let $\left(\mathcal{S}^{\prime \prime}, \beta^{\prime}\right)$ be a labeled composition of $\left(\mathcal{S}^{\prime}, \beta^{\prime}\right)$ with $(\mathcal{S}, \beta)$, and let $\left(\iota, \iota_{\mathrm{Fl}}\right)$ be a labeled section. The image of $\mathcal{S}$ under the section is a subgraph $\Gamma=\left(V, \mathrm{Fl} ; \mathrm{pr}, \varrho_{\Gamma}\right)$ of $\mathcal{S}^{\prime \prime}$, where $\varrho_{\Gamma} \equiv \varrho$ whenever $\varrho^{\prime \prime} \circ=\iota \circ \varrho$, and otherwise $\varrho_{\Gamma}=i d$. Then, the contracted components are by definition the components of the complement $\left(\mathcal{S}^{\prime \prime} \backslash \Gamma,\left.\beta^{\prime}\right|_{\mathcal{S}^{\prime \prime} \backslash \Gamma}\right)$. In this situation, we will say that $(\mathcal{S}, \beta)$ is the contraction of $\left(\mathcal{S}^{\prime \prime}, \beta^{\prime}\right)$.

In Figure 1, the black spots in the graph of $\mathcal{S}^{\prime \prime}$ represent the image under a section of the vertices of $\mathcal{S}$. The contracted components, there are four of them, are the complement of the stars at the black vertices; hence they are given by the connected chains of white vertices together with their halfedges.

Finally, we introduce the notion of stability of graphs. This will be particularly relevant in the case the graphs are represented by stable maps. Let $\mathcal{S}$ be a forest. A vertex $v \in V$ is said to be stable if $|v| \geq 3$. The forest $\mathcal{S}$ is said to be stable if all its vertices are stable. Given a $B$-labeling $\beta$ on $\mathcal{S}$ we say that a vertex $v \in V$ is $B$-stable if either $\beta(v) \neq 0$ or $v$ is stable. The labeled graph $(\mathcal{S}, \beta)$ is $B$-stable when all its vertices are $B$-stable. In this language, a $\{0\}$-stable labeled graph is simply a stable graph.

Observe that stability depends on the given labeling. For instance, if we have a $B^{\prime}$-labeled stable tree $\left(\mathcal{S}^{\prime \prime}, \beta^{\prime}\right)$ and a homomorphism $\pi: B^{\prime} \rightarrow B$ of semi-groups, the induced $B$-labeled graph $\left(\mathcal{S}^{\prime \prime}, \pi \circ \beta^{\prime}\right)$ may not be stable ( since $\pi$ may not be injective). If it is not $B$-stable, we may try to stabilize the tree by inductively contracting all the unstable vertices until the remaining vertices are all $B$-stable. By contraction of an unstable vertex $v \in V^{\prime \prime}$ of $\mathcal{S}^{\prime \prime}$ we mean the following. First, we delete the star at $v$, possibly creating one or two tails (since $|v| \leq 2$ ). If $v$ has no edge, then the tree $\mathcal{S}^{\prime \prime}$ disappears. If $v$ has one edge and no tail, then we also delete the created tail. If $v$ has an edge and a tail, then you do not delete the new tail created on its neighbor. If $v$ has two edges, we replace the deleted vertex by an edge obtained from 
gluing together the two created tails. This operation is called elementary contraction. At the end of the inductive procedure, we either end up with a stable tree or with one vertex with less than two tails implying that there were less than two tails in $\mathcal{S}^{\prime \prime}$ to begin with. The reason for this is that elementary contractions preserve connectedness of the graph and do not change the number of tails unless $\mathcal{S}^{\prime \prime}$ had less than two tails to begin with. This is essentially the content of the first statement of the following standard lemma:

Lemma 4.1. Let $\pi: B^{\prime} \rightarrow B$ be a morphism of semi-groups. Suppose $\left(\mathcal{S}^{\prime \prime}, \beta^{\prime}\right)$ is a stable $B^{\prime}$-labeled tree, then we have one of the following: (1) there exist a stable tree $(\mathcal{S}, \beta)$ and a stable forest $\left(\mathcal{S}^{\prime}, \beta^{\prime}\right)$ such that $\left(\mathcal{S}^{\prime \prime}, \pi \circ \beta^{\prime}\right)$ is their $\pi$-composition; (2) $\mathcal{S}^{\prime \prime}$ has no more than two tails. Moreover, in case (1), there is a natural labeled section of the composition.

Proof. the tree $\mathcal{S}$ is obtained from the construction in the paragraph preceding the lemma. The labeling $\beta$ is simply the restriction of $\pi \circ \beta^{\prime}$ to the vertices of $\mathcal{S}^{\prime \prime}$ remaining after the stabilization procedure. Also, from the construction of $(\mathcal{S}, \beta)$ we have natural inclusions $\iota: V \hookrightarrow V^{\prime \prime}$ and $\iota_{\mathrm{Fl}}: \mathrm{Fl} \hookrightarrow \mathrm{Fl}^{\prime \prime}$. These inclusions provide the desired labeled section in the last statement.

Now, suppose $(\mathcal{S}, \beta)$ is stable. We describe the forest $\left(\mathcal{S}^{\prime}, \beta^{\prime}\right)$. By construction, $\mathcal{S}^{\prime}$ has the same set of vertices, the same set of half-edges and the same vertex assignment map as $\mathcal{S}^{\prime \prime}$. More formally, $\mathcal{S}^{\prime}=\left(V^{\prime \prime}, \mathrm{Fl}^{\prime \prime} ; \mathrm{pr}^{\prime \prime}, \varrho^{\prime}\right)$. The involution $\varrho^{\prime}$ remains to be described. First, observe that $\varrho^{\prime}$ coincides with $\varrho^{\prime \prime}$ on every edge of $\mathcal{S}^{\prime}$, since any edge of $\mathcal{S}^{\prime}$ is also an edge of $\mathcal{S}^{\prime \prime}$. Finally, we set $\varrho^{\prime}(f)=f$ on each tail $f$ of $\mathcal{S}^{\prime}$, since each tail of $\mathcal{S}^{\prime}$ must be a fixed point of the involution.

The natural section mentioned in the proof will be called $\pi$-section.

Definition 4.1. Let $\pi: B^{\prime} \rightarrow B$ be a homomorphism of semi-groups. We say that the tree $(\mathcal{S}, \beta)$ above is the $\pi$-stabilization of $\left(\mathcal{S}^{\prime \prime}, \beta^{\prime}\right)$ and we denote it $S_{\pi}\left(\mathcal{S}^{\prime \prime}, \beta^{\prime}\right)$. The vertices in $\mathcal{S}$ are called $\pi$-stable, and the contracted components are called $\pi$-unstable.

Let $(\mathcal{S}, \beta)=S_{\pi}\left(\mathcal{S}^{\prime \prime}, \beta^{\prime}\right)$. The vertices in the image of the section are said to be $B$-stable. Now, let $c$ be one of the contracted components in $\mathcal{S}^{\prime \prime}$. Then $c$ is a tree with at most 2 tails. A tail of $c$ is called exterior if it is also a tail of $\mathcal{S}^{\prime \prime}$. Otherwise, it is called interior. In the case $c$ has 1 interior tail, we say that $c$ is a $(\pi-)$ contracted branch. In the case $c$ has two interior tails, we say that $c$ is a $(\pi-)$ connecting branch. For a connecting branch there is a unique path of vertices connecting the 2 tails. This path forms a connecting chain.

Example 4.2. Consider Figure 1 above. Let $\beta$ be the $\{0\}$-labeling on $\mathcal{S}$, and let $\beta^{\prime}$ be a $\mathbb{Z}_{2}$-labeling on $\mathcal{S}^{\prime}$ defined by $\beta^{\prime} \equiv 1(\bmod 2)$. Obviously, $\beta=\pi \circ \beta^{\prime}$ for any morphism $\pi: \mathbb{Z}_{2} \rightarrow\{0\}$. Note that $\left(\mathcal{S}^{\prime \prime}, \beta^{\prime}\right)$ is stable but 
$\left(\mathcal{S}^{\prime \prime}, \beta\right)$ is not. In fact, $(\mathcal{S}, \beta)$ is the $\pi$-stabilization of $\left(\mathcal{S}^{\prime \prime}, \beta^{\prime}\right)$. The contracted component given by the white vertex lying between the two black vertices is a $\pi$-connecting branch. The other three contracted components define $\pi$-contracted branches.

4.1.2. Moduli space of stable maps. The discussion below is taken from [17], Chapter 5, and [21], where the proofs of all the claims can be found. Denote by $\overline{\mathcal{M}}_{g, l}$ the Deligne-Mumford moduli space of stable curves with genus $g$ and $l$-marked points. We shall only consider the case $g=0$. Points in $\overline{\mathcal{M}}_{0, l}$ are given by isomorphism classes of elements,

$$
\mathbf{j} \equiv\left(\Sigma, \mathbf{j}, \mathbf{x}:=\left(x_{1}, \ldots, x_{l}\right)\right),
$$

where $(\Sigma, \mathbf{j})$ is a nodal Riemann surface of arithmetic genus 0 with no selfintersection, together with $l$ pairwise disjoint marked points on $\Sigma$ denoted $\mathbf{x}$, which are disjoint from the nodes. Furthermore, each component of $\Sigma$ has at least three special points (i.e., marked points or nodes). Two pointed nodal curves, $(\Sigma, \mathbf{j}, \mathbf{x})$ and $\left(\Sigma^{\prime}, \mathbf{j}^{\prime}, \mathbf{x}^{\prime}\right)$, are isomorphic if there is a diffeomorphism $\varphi: \Sigma \rightarrow \Sigma^{\prime}$ satisfying:

$$
\varphi^{*} \mathbf{j}^{\prime}=\mathbf{j}, \quad \text { and } \quad \varphi\left(x_{i}\right)=x_{i}^{\prime} .
$$

We denote by $\operatorname{Aut}(\Sigma, \mathbf{j}, \mathbf{x})$ the automorphisms of $(\Sigma, \mathbf{j}, \mathbf{x})$, i.e., the subset of diffeomorphisms of nodal surfaces, $\varphi: \Sigma \rightarrow \Sigma$, such that $\varphi^{*} \mathbf{j}=\mathbf{j}$ and $\varphi(\mathbf{x})=\mathbf{x}$. This group is invariant under isomorphism of nodal curves. Also, it is standard that the elements of $\overline{\mathcal{M}}_{0, l}$ are automorphism free.

Let $\overline{\mathcal{M}}_{0, l}(X, A, J)$ denote the compactified moduli space of stable $J$-holomorphic maps from (nodal) curves of genus 0 with $l$ marked points into the symplectic manifold $X$, representing the class $A \in H_{2}(X, \mathbb{Z})$. Points in $\overline{\mathcal{M}}_{0, l}(X, A, J)$ are given by isomorphism classes of parameterized stable pseudo-holomorphic maps $(\mathbf{j}, u) \equiv((\Sigma, \mathbf{j}, \mathbf{x}), u)$, where $(\Sigma, \mathbf{j}, \mathbf{x})$ is a Riemann nodal curve of genus 0 with $l$ marked points (not necessarily stable), and $u: \Sigma \rightarrow X$ is $(\mathbf{j}, J)$-holomorphic and such that each component on which $u$ is constant has at least three special points. We say that $(\mathbf{j}, u)$ is isomorphic to $\left(\mathbf{j}^{\prime}, u^{\prime}\right)$ if there is an isomorphism of pointed nodal curve, $\varphi$, between $\mathbf{j}$ and $\mathbf{j}^{\prime}$ such that $\varphi^{*} u=u^{\prime}$. Let $\operatorname{Aut}(\mathbf{j}, u)$ denote the corresponding automorphism group. It is well known that stability implies finiteness of the automorphism groups. Moreover, if the map $u$ is reduced, or simple in the sense that $(\mathbf{j}, u)$ has no ramified component or any two component having the same non-constant image in $X$, then $\operatorname{Aut}(\mathbf{j}, u)=i d$; when the map $u$ has only one component, this notion of simple map coincides with the notion of somewhere injective map meaning that there exists a point $z_{0} \in S^{2}$ such that $d u\left(z_{0}\right)$ is injective and $u^{-1}\left(u\left(z_{0}\right)\right)=z_{0}$. It is well known that any stable pseudo-holomorphic map can be reduced to a simple stable map. The reduction process however changes the homology class of the map ([17], Proposition 6.1.2.). 
The moduli spaces $\overline{\mathcal{M}}_{0, l}$ and $\overline{\mathcal{M}}_{0, l}(X, A, J)$ are stratified, with strata labeled by stable labeled trees of genus 0 called stable stratum data of the strata, or combinatorial type. For a tree $\mathcal{S}=(V, \mathrm{Fl} ; \mathrm{pr}, \varrho)$, the set $V$ corresponds to the components of $(\Sigma, \mathbf{j})$, while $\mathrm{Fl}$ corresponds to the set of special points on the curve. For $\overline{\mathcal{M}}_{0, l}(X, A, J)$ we have a $H_{2}(X, \mathbb{Z})$-labeling giving the homology class represented by the image of each component in $X$. Note that $\overline{\mathcal{M}}_{0, l}$ and $\overline{\mathcal{M}}_{0, l}(X, A, J)$ coincide when $X=p t$ and $A=0$. A strata with stratum data $\mathcal{S}$ will be denoted $\mathcal{M}_{\mathcal{S}}$ or $\mathcal{M}_{\mathcal{S}}(X, J)$, and $\mathcal{M}_{\mathcal{S}}^{*}(X, J)$ will denote the subset of $\mathcal{M}_{\mathcal{S}}(X, J)$ consisting of simple stable maps. Furthermore, the partial order $\prec$ on labeled graphs induces inclusions of strata in the sense that $\mathcal{M}_{\mathcal{S}^{\prime}} \subset \overline{\mathcal{M}}_{\mathcal{S}}$ if and only if $\mathcal{S}^{\prime} \prec \mathcal{S}$, and similarly for $\mathcal{M}_{\mathcal{S}}(X, J)$. Note that there are finitely many strata in the compactified moduli space since for fixed $l$ and $A$, the set of possible combinatorial types for genus 0 stable map with $l$ markings representing $A$ is finite.

As we are only considering genus 0 stable maps, we can in fact fix the complex structure on each component of the nodal surface to be the standard one. Let $\widetilde{\mathcal{M}}_{\mathcal{S}}(X, J)$ denote the moduli space of parameterized stable $J$-holomorphic maps $(\Sigma, u, \mathbf{x})$ representing $\mathcal{S}$, and let $G_{\mathcal{S}}$ be the reparameterization group of the domain $(\Sigma, \mathbf{x})$. The stratum $\mathcal{M}_{\mathcal{S}}(X, J)$ is then identified to the quotient of $\widetilde{\mathcal{M}}_{\mathcal{S}}(X, J)$ under the (proper) action of $G_{\mathcal{S}}$. We describe it for the reader's convenience. First, note that a stable map, $(\Sigma, u, \mathbf{x}) \in \widetilde{\mathcal{M}}_{\mathcal{S}}(X, J)$, is determined by a triple, $(u, \mathbf{y}, \mathbf{x})$, where $\mathbf{y}:=\left\{y_{v v^{\prime}}\right\}_{v E v^{\prime}}$ for $v, v^{\prime} \in V$, is the data given by the nodal points in $\Sigma$. Let $\Sigma_{v}$ denote the component of $\Sigma$ corresponding to $v \in V$, and let $u_{v}$ be the restriction of $u$ to $\Sigma_{v}$. The group $G_{\mathcal{S}}$ consists of pairs $\left(\left\{\varphi_{v}\right\}_{v \in V}, \gamma\right)$, where $\gamma \in \operatorname{Aut}(\mathcal{S})$ is a tree-with-tails automorphism, and $\varphi_{v}: \Sigma_{v} \rightarrow \Sigma_{\gamma(v)}$ is an element of $P S L_{2}(\mathbb{C})$. Then, the action of $G_{\mathcal{S}}$ on $\widetilde{\mathcal{M}}_{\mathcal{S}}(X, J)$ is given by

$$
\left(\left\{\varphi_{v}\right\}, \gamma\right) \cdot(u, \mathbf{y}, \mathbf{x}):=\left(\left\{u_{v} \circ \varphi_{v}^{-1}\right\},\left\{\varphi_{v}\left(y_{v v^{\prime}}\right)\right\}_{v E v^{\prime}},\left\{\varphi_{p\left(x_{k}\right)}\left(x_{k}\right)\right\}_{k \in\{1, \ldots, l\}}\right)
$$

Before carrying out the description of stable $J_{P}$-holomorphic maps in a Hamiltonian fibration $\pi: P \rightarrow B$, we first make sure that we have the appropriate energy bounds in order to apply Gromov's compactness ([7] Chapter V, [17] Theorem 5.5.5, [21] Proposition 3.1.).

4.1.3. Energy identities. Suppose $J_{P}$ is a fibered structure obtained from a connection $\tau$, an element $J_{B} \in \mathcal{J}_{B}$ and a family $J \in \mathcal{J}^{V}$, and let $g_{J_{P}}$ be the corresponding split metric on $P$. For a smooth map $u: S^{2} \rightarrow P$, we define its total energy to be its Dirichlet norm with respect to $g_{J_{P}}$ :

$$
E(u):=\frac{1}{2} \int_{S^{2}}\|d u\|_{g_{J_{P}}}^{2} d \operatorname{vol}_{S^{2}}
$$


Since $g_{J_{P}}$ is split, $E(u)$ can be written as the sum,

$$
\frac{1}{2} \int_{S^{2}}\|d(\pi(u))\|_{g_{J_{B}}}^{2} d \operatorname{vol}_{S^{2}}+\frac{1}{2} \int_{S^{2}}\left\|(d u)^{v}\right\|_{g_{J}}^{2} d \operatorname{vol}_{S^{2}}:=E_{B}(\pi(u))+E^{\mathrm{vert}}(u),
$$

where $E_{B}(\pi(u))$ is the energy of $u_{B}:=\pi(u)$ (with respect to $J_{B}$ ), and $E^{\text {vert }}(u)$ is the vertical energy. When $u$ is $J_{P}$-holomorphic, it turns out that:

$$
E_{B}\left(u_{B}\right)=\int_{S^{2}} u_{B}^{*} \omega_{B}, \quad \text { and } \quad E^{\mathrm{vert}}(u)=\int_{S^{2}} u^{*} \tau+\int_{S^{2}} R(u) d \operatorname{vol}_{S^{2}},
$$

where the second identity is obtained since:

$$
\tau\left(d u, J_{P} d u\right)=\omega\left(d u^{v}, J d u^{v}\right)-R\left(d u^{h}, J_{P} d u^{h}\right) .
$$

Consider the Hofer norm of the symplectic curvature,

$$
\|R\|_{H}:=\int_{B}\left(\max _{p \in F_{b}} R(p)-\min _{p \in F_{b}} R(p)\right) \omega_{B}^{n_{B}},
$$

which is bounded by compactness of $P$. We obtain the upper bound:

Lemma 4.2. For every $J_{P}$-holomorphic map u:

$$
E(u) \leq \int_{S^{2}} u^{*} \tau+\|R\|_{H}+\int_{S^{2}} u_{B}^{*} \omega_{B}
$$

Applying Gromov's compactness, we conclude that any sequence of simple $J_{P}$-holomorphic map representing $\sigma$ must converge (up to taking a subsequence) to a stable $J_{P}$-holomorphic map. From the upper bound 4.1 and the stability condition for stable maps, we deduce the following result that is analogous to Lemma 4.5 in [21]:

Lemma 4.3. Let $\mathcal{D}_{0, l}^{\sigma}$ denote the set of possible combinatorial types for genus 0 stable map with $l$ markings representing $\sigma$. Then $\left|\mathcal{D}_{0, l}^{\sigma}\right|<\infty$.

4.1.4. Forgetful maps and Hamiltonian fibrations. The natural map, $\pi_{p t}: X \rightarrow p t$, induces the map $\pi_{p t_{*}}: H_{2}(X, \mathbb{Z}) \rightarrow\{0\}$ on the labeling groups. We denote by $S_{\pi_{p t_{*}}}$ the corresponding $\pi_{p t_{*}}$-stabilization map on labeled graphs defined in Definition 4.1. The forgetful map is the map:

$$
\bar{\pi}_{p t}: \overline{\mathcal{M}}_{0, l}(X, A) \rightarrow \overline{\mathcal{M}}_{0, l}, \quad \bar{\pi}_{p t}(\mathbf{j}, u)=\mathbf{j}^{\mathrm{st}},
$$

where $\mathbf{j}^{\text {st }}$ denotes the stabilization of $\mathbf{j}$, that is $\mathbf{j}^{\text {st }}$ is obtained by contracting the unstable components recursively. It is well known that, when restricted to strata, the forgetful map defines maps:

$$
\bar{\pi}_{p t}^{\mathcal{S}}: \mathcal{M}_{\mathcal{S}}(X) \rightarrow \mathcal{M}_{S_{\pi_{p t_{*}}}(\mathcal{S})} .
$$

This procedure can be generalized to any Hamiltonian fibration $\pi$ : $P \rightarrow B$ with coupling form $\tau$. Again, the moduli spaces of stable pseudoholomorphic maps in $P$ and $B$ are stratified, with strata labeled by stable labeled trees. Note that $\pi$ induces a map $\pi_{*}: H_{2}(P, \mathbb{Z}) \rightarrow H_{2}(B, \mathbb{Z})$ between 
the labeling groups. Let $J_{P}$ be a $(\pi, \tau)$ compatible almost complex structure on $P$ projecting on $J_{B}$. Given a stable stratum data $\mathcal{S}_{P}$ for $J_{P}$-holomorphic maps in $P$ representing $\sigma \in H_{2}(P, \mathbb{Z})$, we have that $S_{B}:=S_{\pi_{*}}\left(\mathcal{S}_{P}\right)$ is a stable stratum data for $\overline{\mathcal{M}}_{0, l}\left(B, \pi_{*}(\sigma), J_{B}\right)$, and we have a $\pi$-forgetful map:

$$
\bar{\pi}_{\mathcal{S}_{P}}: \mathcal{M}_{\mathcal{S}_{P}}\left(P, J_{P}\right) \rightarrow \mathcal{M}_{\mathcal{S}_{B}}\left(B, J_{B}\right), \quad(\mathbf{j}, u) \rightarrow\left(\mathbf{j}^{\mathrm{st}, \pi}, u_{B}:=\pi(u)\right),
$$

where $\mathbf{j}^{\text {st }, \pi}$ is the Riemann nodal surface consisting of the components determined by the $\pi_{*}$-section of the $\pi_{*}$-stabilization of labeled graphs (which has combinatorial type $S_{\pi_{*}}\left(\mathcal{S}_{P}\right)$ ), and $u_{B}$ restricts to the $\pi_{*}$-stable components. We further have a reparameterization-group-equivariant map:

$$
\pi_{\mathcal{S}_{P}}: \widetilde{\mathcal{M}}_{\mathcal{S}_{P}}\left(P, J_{P}\right) \rightarrow \widetilde{\mathcal{M}}_{\mathcal{S}_{B}}\left(P, J_{B}\right), \quad(u, \mathbf{y}, \mathbf{x}) \mapsto\left(u_{B}, \mathbf{y}_{B}:=\mathbf{y}^{\mathrm{st}, \pi}, \mathbf{x}\right)
$$

lifting $\bar{\pi}_{\mathcal{S}_{P}}$. Note that, a priori, $\bar{\pi}_{\mathcal{S}_{P}}$ may not respect simplicity of pseudoholomorphic stable maps (e.g., by sending a simple $J_{P}$-holomorphic map to a multiply covered $J_{B}$-holomorphic map). This has dramatic effects regarding transversality within the range of fibered almost complex structures. For each stable stratum data $\mathcal{S}_{P}$, we will consequently restrict our attention to the subset, $\mathcal{M}_{\mathcal{S}_{P}}^{* *}\left(P, J_{P}\right)$, of simple stable elements in $\mathcal{M}_{\mathcal{S}_{P}}\left(P, J_{P}\right)$ lying in the preimage of $\mathcal{M}_{\mathcal{S}_{B}}^{*}\left(B, J_{B}\right)$ under $\bar{\pi}_{\mathcal{S}_{P}}$. In particular, we denote by $\left(\pi_{\mathcal{S}_{P}}^{-1}\left(\bar{u}_{B}\right)\right)^{*}$ the set of simple parameterized stable pseudo-holomorphic maps lying in the fiber of $\pi_{\mathcal{S}_{P}}$ above $\bar{u}_{B} \in \widetilde{\mathcal{M}}_{\mathcal{S}_{B}}^{*}\left(B, J_{B}\right)$, and we use the notation $\left(\bar{\pi}_{\mathcal{S}_{P}}^{-1}\left(\bar{u}_{B}\right)\right)^{*}$ to denote the corresponding quotient under reparameterizations.

4.2. Transversality on every strata. We begin by recalling some standard notations and facts concerning transversality for a symplectic manifold $(X, \omega)$. Then, we apply it to Hamiltonian fibrations. Finally, we formulate the corresponding cobordism invariance.

4.2.1. The non-fibered case. Let $\mathcal{S}_{X}=(V, \mathrm{Fl} ; \mathrm{pr}, \varrho)$ be a stable stratum data for $\overline{\mathcal{M}}_{0, l}(X, A, J)$ with homological labeling $\beta$. For every $v \in V$, let $\sigma_{v}:=\beta(v)$, and set

$$
\widetilde{\mathcal{M}}{ }^{*}\left(X, \beta, \mathcal{J}_{X}\right):=\left\{\left(u:=\left\{u_{v}\right\}_{v \in V}, J\right) \mid J \in \mathcal{J}_{X} \quad \text { and } \quad u_{v} \in \widetilde{\mathcal{M}}^{*}\left(X, \sigma_{v}, J\right)\right\} .
$$

This defines a subset of

$$
\mathcal{B}_{X}^{1, p}\left(\beta, \mathcal{J}_{X}\right):=\prod_{v \in V} \mathcal{B}_{X}^{1, p}\left(\sigma_{v}\right) \times \mathcal{J}_{X}
$$

We describe $\widetilde{\mathcal{M}}_{\mathcal{S}_{X}}^{*}(X, J)$ as a subset of $\widetilde{\mathcal{M}}^{*}\left(X, \beta, \mathcal{J}_{X}\right) \times I\left(\mathcal{S}_{X}\right)$, where the incidental subvariety $I\left(\mathcal{S}_{X}\right)$ is the subset of tuples

$$
(\mathbf{y}, \mathbf{x}):=\left(\left\{y_{v v^{\prime}}\right\}_{v E v^{\prime}}, x_{1}, \ldots, x_{l}\right) \in\left(S^{2}\right)^{2|E|} \times\left(S^{2}\right)^{|V|}
$$


such that for every $v \in V$ the points, $y_{v v^{\prime}}$ with $v E v^{\prime}$, and $x_{m}$ with $\operatorname{pr}\left(x_{m}\right)=$ $v$, are disjoint. First, to each edge of the graph $\mathcal{S}_{X}$ we associate a copy of the diagonal $\Delta_{X} \subset X^{2}$ and set the edge diagonal $\Delta_{E} \subset X^{2|E|}$ to be the product of these diagonals over the set $E$ of all edges. We have a natural map

$$
e v_{E}: \widetilde{\mathcal{M}}^{*}\left(X, \beta, \mathcal{J}_{X}\right) \times I\left(\mathcal{S}_{X}\right) \rightarrow(X)^{2|E|},
$$

called universal edge evaluation map, assigning to each pair $(u, J, \mathbf{y}, \mathbf{x})$ the corresponding "evaluation at the nodes":

$$
u(\mathbf{y}):=\left\{\left(u_{v}\left(y_{v v^{\prime}}\right), u_{v^{\prime}}\left(y_{v^{\prime} v}\right)\right)\right\}_{v E v^{\prime}} .
$$

The preimage of $\Delta_{E}$ under $e v_{E}$ is the (parameterized) universal moduli space denoted $\widetilde{\mathcal{M}}_{\mathcal{S}_{X}}^{*}\left(X, \mathcal{J}_{X}\right)$. Then, it is easy to see that

$$
\widetilde{\mathcal{M}}_{\mathcal{S}_{X}}^{*}(X, J)=\left(p^{\mathcal{J}_{X}}\right)^{-1}(J) \cap \widetilde{\mathcal{M}}_{\mathcal{S}_{X}}^{*}\left(X, \mathcal{J}_{X}\right)
$$

where $p^{\mathcal{J}_{X}}$ denotes the projection from $\widetilde{\mathcal{M}}^{*}\left(X, \beta, \mathcal{J}_{X}\right) \times I\left(\mathcal{S}_{X}\right)$ to $\mathcal{J}_{X}$. The standard transversality theorem asserts the following:

Theorem 4.1 ([17] Theorem 6.2.6, [21] Theorem 4.7). There is a subset $\mathcal{J}_{X, \text { reg }}\left(\mathcal{S}_{X}\right)$ of second category in $\mathcal{J}_{X}$, such that for each $J \in$ $\mathcal{J}_{X, \text { reg }}\left(\mathcal{S}_{X}\right)$ the moduli space $\mathcal{M}_{\mathcal{S}_{X}}^{*}(X, J)$ is a smooth oriented manifold of real dimension:

$$
\operatorname{dim}\left(\mathcal{M}_{\mathcal{S}_{X}}^{*}(X, J)\right)=2 n+2 \sum_{v \in V} c_{1}^{T X}\left(\sigma_{v}\right)+2 l-2|E|-6 .
$$

The set $\mathcal{J}_{X \text {,reg }}\left(\mathcal{S}_{X}\right)$ of regular $\omega$-tame almost complex structure for $\mathcal{S}_{X}$ is explicitly given by the following conditions:

(i) for every $v \in V$, for every $u \in \widetilde{\mathcal{M}}^{*}\left(X, \sigma_{v}, J\right)$, the linearization $D_{u}^{X}$ of $\bar{\partial}_{J}$ at $u$ is surjective.

(ii) the restriction of $e v_{E}$ to $\widetilde{\mathcal{M}}_{\mathcal{S}_{X}}^{*}(X, J)$ is transversal to $\Delta_{E}$.

It follows from (i) that for regular $J$ the moduli spaces $\widetilde{\mathcal{M}}{ }^{*}\left(X, \sigma_{v}, J\right)$ are naturally oriented manifolds of dimension $\operatorname{Ind}\left(D^{X}\right)=2 n+2 c_{1}^{T X}\left(\sigma_{v}\right)$. Point (ii) then implies that $\widetilde{\mathcal{M}}_{\mathcal{S}_{X}}^{*}(X, J)$ is a smooth oriented manifold. Since the $6(|E|+1)$-dimensional group $G_{\mathcal{S}_{X}}$ acts freely and properly by orientation preserving diffeomorphisms on this latter manifold, it follows that $\mathcal{M}_{\mathcal{S}_{X}}^{*}(X, J)$ is a smooth oriented manifold of the stated dimension.

We briefly sketch the idea of proof for the genericity of $\mathcal{J}_{X \text {,reg }}\left(\mathcal{S}_{X}\right)$. We refer to [17] (Chapters 3 and 6) for the details. The main idea is to show that the universal moduli space is a separable Banach manifold and that $p^{\mathcal{J}_{X}}$ is a Fredholm map between separable Banach manifolds in order to apply Sard-Smale. Of course, this does not apply straighforwardly here since, for instance, $\mathcal{J}_{X}$ is not Banach. Instead, we consider the set $\mathcal{J}_{X}^{r}$ of $\omega$-tame 
almost complex structure of class $C^{r}$ with $r \geq 2$. Now, set

$$
\mathcal{E}_{X}^{p, r}(\beta):=\bigsqcup_{(u, J) \in \mathcal{B}_{X}^{1, p}\left(\beta, \mathcal{J}_{X}^{r}\right)}\left(\bigoplus_{v \in V} \mathcal{E}_{X, u_{v}}^{p}(J)\right) .
$$

By a standard argument $\mathcal{E}_{X}^{p, r}(\beta)$ is a locally trivial $C^{r-1}$ Banach fibration over $\mathcal{B}_{X}^{1, p}\left(\beta, \mathcal{J}_{X}^{r}\right)$. This Banach fibration admits the section

$$
s_{X}:(u, J) \mapsto \bar{\partial}_{J}(u),
$$

and clearly $\widetilde{\mathcal{M}}^{*}\left(X, \beta, \mathcal{J}_{X}^{r}\right)$ is a subset of $s_{X}^{-1}(0)$. Moreover, the linearization $\widetilde{D}_{(u, J)}^{X}$ of $s_{X}$ at $(u, J)$ is given by:

$$
\begin{aligned}
\widetilde{D}_{(u, J)}^{X}: \bigoplus_{v \in V} \mathcal{X}_{X, u_{v}}^{1, p} \oplus T_{J} \mathcal{J} & \longrightarrow \bigoplus_{v \in V} \mathcal{E}_{X, u_{v}}^{p}(J) \\
(\xi, Y) & \mapsto D_{u}^{X} \xi+\frac{1}{2} Y \cdot(d u) \cdot j_{0} .
\end{aligned}
$$

One can further show that this operator is onto for every $(u, J)$. It follows that $\widetilde{\mathcal{M}}^{*}\left(X, \beta, \mathcal{J}_{X}^{r}\right)$ is a separable Banach manifold. One concludes that $\widetilde{\mathcal{M}}^{*}\left(X, \mathcal{J}_{X}^{r}\right)$ is a Banach manifold by showing that the edge evaluation map $e v_{E}$ is transversal to $\Delta_{E}$. This is done recursively on the set of all labeled forest, the induction argument being made on the number of edges of the forests. Finally, a simple computation shows that $p^{\mathcal{J}_{X}^{r}}$ is Fredholm with the same kernel and cokernel as the linearized operator $\widetilde{D}^{X}$. Thus, by SardSmale (when $r$ is big enough) one obtains a generic subset $\mathcal{J}_{X \text {,reg }}^{r}\left(\mathcal{S}_{X}\right) \subset \mathcal{J}_{X}^{r}$, which in fact coincides with the regularizing set defined above, but with $C^{r}$ elements only. One concludes in the $C^{\infty}$ case by an argument due to Taubes (see [17], Chapter 3, Proof of Theorem 3.1.5).

4.2.2. The Hamiltonian fibration case. Let $\pi: P \rightarrow B$ be a Hamiltonian fibration with coupling form $\tau$. We fix some notations before reformulating Theorem B and giving its proof.

Fix a stable stratum data $\mathcal{S}_{P}=(V, \mathrm{Fl} ; \mathrm{pr}, \varrho)$ with homological labeling $\beta_{P}$, and set $\mathcal{S}_{B}=S_{\pi_{*}}\left(\mathcal{S}_{P}\right)=\left(V_{B}, \mathrm{Fl}_{B} ; \mathrm{pr}_{B}, \varrho_{B}\right)$ with corresponding labeling $\beta_{B}$. Let $E$ and $E_{B}$ denote the corresponding set of edges. We will assume below that the homomorphism $\beta_{B}^{\prime}:=\pi_{*} \circ \beta_{P}$ is non-zero, thus forcing $\beta_{B}$ to be non-zero. Set $\sigma_{v}:=\beta_{P}(v)$.

Let $\widetilde{\mathcal{M}}^{* *}\left(P, \beta_{P}, \mathcal{J}_{P}\right)$ be the restriction of $\widetilde{\mathcal{M}}^{*}\left(P, \beta_{P}, \mathcal{J}_{P}\right)$ to simple maps having simple projection under $\pi$ and let

$$
\widetilde{\mathcal{M}}_{\mathcal{S}_{P}}^{* *}\left(P, \mathcal{J}_{P}\right):=e v_{E}^{-1}\left(\Delta_{E}\right)
$$

be the corresponding universal moduli space. Similarly to the general case, we say that $J_{P} \in \mathcal{J}_{P, \text { reg }}\left(\mathcal{S}_{P}\right)$ if and only if: (i) $\forall v \in V, \quad \forall u \in$ 
$\widetilde{\mathcal{M}}^{* *}\left(P, \sigma_{v}, J_{P}\right)$, the operator $D_{u}^{P}$ is onto; (ii) the restriction of $e v_{E}$ to $\widetilde{\mathcal{M}}_{\mathcal{S}_{P}}^{* *}(P, J)$ is transversal to $\Delta_{E}$.

We have a natural map

$$
\begin{aligned}
\Pi_{\mathcal{S}_{P}}: \widetilde{\mathcal{M}}^{* *}\left(P, \beta_{P}, \mathcal{J}_{P}\right) \times I\left(\mathcal{S}_{P}\right) & \rightarrow \widetilde{\mathcal{M}}^{*}\left(B, \beta_{B}, \mathcal{J}_{B}\right) \times I\left(\mathcal{S}_{B}\right) \\
\left(u, J_{P}, \mathbf{y}, \mathbf{x}\right) & \mapsto\left(u_{B}, J_{B}, \mathbf{y}_{B}, \mathbf{x}\right),
\end{aligned}
$$

induced from $\pi$ and the projection $p_{1}: \mathcal{J}_{P} \rightarrow \mathcal{J}_{B}$. In fact, the restriction of $\Pi_{\mathcal{S}_{P}}$ to $\widetilde{\mathcal{M}}_{\mathcal{S}_{P}}^{* *}\left(P, \mathcal{J}_{P}\right)$ induces a map between universal moduli spaces:

$$
\tilde{\pi}_{\mathcal{S}_{P}}:=\pi_{\mathcal{S}_{P}} \times p_{1}: \widetilde{\mathcal{M}}_{\mathcal{S}_{P}}^{*}\left(P, \mathcal{J}_{P}\right) \rightarrow \widetilde{\mathcal{M}}_{\mathcal{S}_{B}}^{*}\left(B, \mathcal{J}_{B}\right) .
$$

Since the edge evaluation maps commute with $\widetilde{\pi}_{\mathcal{S}_{P}}$ and the projection from $P^{2|E|}$ to $B^{2\left|E_{B}\right|}$, it follows easily that if $J_{P}$ is regular for $\mathcal{S}_{P}$ then $J_{B}$ is regular for $\mathcal{S}_{B}$.

Now fix a regular $J_{B}$. Also, let $p_{23}: \mathcal{J}_{P} \rightarrow \mathcal{J}^{V} \times \mathcal{H}$ denote the obvious projection. Note that

$$
\left(\bar{\pi}_{\mathcal{S}_{P}}^{-1}\left(\bar{u}_{B}\right)\right)^{*}=\left(p_{23} \circ p^{\mathcal{J}_{P}}\right)^{-1}(J, H) \cap \Pi_{\mathcal{S}_{P}}^{-1}\left(u_{B}, J_{B}\right) \cap e v_{E}^{-1}\left(\Delta_{E} \cap F^{2|E|}\right) .
$$

A pair $(J, H) \in \mathcal{J}^{V} \times \mathcal{H}$ turning this intersection into an oriented manifold is said to be fiber regularizing. More precisely, a pair $(J, H)$ is fiber regularizing for $\left(\bar{\pi}_{\mathcal{S}_{P}}^{-1}\left(\bar{u}_{B}\right)\right)^{*}$ if for every $\left(u \equiv\left\{u_{v}\right\}_{v \in V}, \mathbf{y}, \mathbf{x}\right) \in\left(\pi_{\mathcal{S}_{P}}^{-1}\left(\bar{u}_{B}\right)\right)^{*}$ the following conditions are satisfied:

(a) $\forall v \in V, \quad \forall u_{v} \in \widetilde{\mathcal{M}}^{* *}\left(P, \sigma_{v}, J_{P}\right)$, the operator $D_{\left(u_{v}, J_{P}\right)}^{v}$ is onto;

(b) the restriction of $e v_{E}$ to $\left(\pi_{\mathcal{S}_{P}}^{-1}\left(\bar{u}_{B}\right)\right)^{*}$ is transversal to $\Delta_{E} \cap F^{2|E|}$.

We will denote by $\mathcal{J} \mathcal{H}_{\mathrm{reg}}\left(\bar{u}_{B}, J_{B}, \mathcal{S}_{P}\right)$ the set of fiber regularizing pairs for $\left(\bar{\pi}_{\mathcal{S}_{P}}^{-1}\left(\bar{u}_{B}\right)\right)^{*}$. It follows from $(a),(b)$, and the exact sequence $(3.4)$, that for a regularizing pair $(J, H)$, the set $\pi_{\mathcal{S}_{P}}^{-1}\left(\bar{u}_{B}\right)^{*}$ is a smooth oriented manifold with dimension:

$$
\operatorname{dim}\left(\pi_{\mathcal{S}_{P}}^{-1}\left(\bar{u}_{B}\right)^{*}\right)=\operatorname{dim} \widetilde{\mathcal{M}}_{\mathcal{S}_{P}}^{* *}\left(P, J_{P}\right)-\operatorname{dim} \widetilde{\mathcal{M}}_{\mathcal{S}_{\mathcal{B}}}^{*}\left(B, J_{B}\right) .
$$

Since we are only considering vertical deformations of the maps, the dimension of the reparameterizations is $6\left(|E|-\left|E_{B}\right|\right)$, thus giving after quotient:

$$
\operatorname{dim}\left(\bar{\pi}_{\mathcal{S}_{P}}^{-1}\left(\bar{u}_{B}\right)\right)^{*}=2 n_{\mathrm{F}}+2 \sum_{v \in V} c^{v}\left(\sigma_{v}\right)-2|E|+2\left|E_{B}\right| .
$$

We give the proof of the following extension of Theorem B to any strata, which can be seen as a mild extension to the Hamiltonian fibration case of Theorem 4.1.

Theorem B'. Let $\mathcal{S}_{P}$ and $\mathcal{S}_{B}$ be as above.

(1) $\mathcal{J}_{P, \text { reg }}\left(\mathcal{S}_{P}\right)$ is of second category in $\mathcal{J}_{P}$.

(2) For any $J_{B} \in \mathcal{J}_{B, \text { reg }}\left(\mathcal{S}_{B}\right)$ and any $\bar{u}_{B} \in \mathcal{M}_{\mathcal{S}_{B}}^{*}\left(B, J_{B}\right)$ the set of regularizing pairs $\mathcal{J H}_{\text {reg }}\left(\bar{u}_{B}, J_{B}, \mathcal{S}_{P}\right)$ is of second category in $\mathcal{J}^{V} \times \mathcal{H}$. 
Proof. The notations used here are the same as the one introduced in Section 4.2.1. Following the guideline given in the preceding section, we show that

$$
\widetilde{\pi}_{\mathcal{S}_{P}}: \widetilde{\mathcal{M}}_{\mathcal{S}_{P}}^{* *}\left(P, \mathcal{J}_{P}^{r}\right) \rightarrow \widetilde{\mathcal{M}}_{\mathcal{S}_{B}}^{*}\left(B, \mathcal{J}_{B}^{r}\right), \quad r \geq 2,
$$

defines a submersion between separated $C^{r-1}$ Banach submanifolds of $\mathcal{B}_{P}^{1, p}\left(\beta_{P}, \mathcal{J}_{P}^{r}\right)$ and $\mathcal{B}_{B}^{1, p}\left(\beta_{B}, \mathcal{J}_{B}^{r}\right)$. By the discussion in Section 4.2.1, we already know that $\widetilde{\mathcal{M}}_{\mathcal{S}_{B}}^{*}\left(B, \mathcal{J}_{B}^{r}\right)$ is a Banach manifold. We will then proceed as follows:

(I) we show that $\widetilde{\mathcal{M}}^{* *}\left(P, \beta_{P}, \mathcal{J}_{P}^{r}\right)$ is a Banach manifold and that the restriction of the natural map,

$$
\mathfrak{p}:=\pi_{\mathcal{S}_{P}} \times p_{1}: \mathcal{B}_{P}^{1, p}\left(\beta_{P}, \mathcal{J}_{P}^{r}\right) \rightarrow \mathcal{B}_{B}^{1, p}\left(\beta_{B}, \mathcal{J}_{B}^{r}\right), \quad\left(u, J_{P}\right) \mapsto\left(\pi(u), J_{B}\right),
$$

to this product moduli space is a smooth submersion onto the moduli space $\widetilde{\mathcal{M}}^{*}\left(B, \beta_{B}, \mathcal{J}_{B}^{r}\right)$.

(II) Assuming $e v_{E}$ is transversal to $\Delta_{E}$, we show that $\widetilde{\pi}_{\mathcal{S}_{P}}$ is a submersion.

(III) We show that for every labeled forest $\mathcal{S}_{P}$ with $l$-tails, the restriction of $d e v_{E}$ to ker $d \Pi_{\mathcal{S}_{P}}$ is transversal to the subspace $\left.T \Delta_{E}\right|_{T F^{E}}$. This implies that $e v_{E}$ is transversal to $\Delta_{E}$ for any labeled forest $\mathcal{S}_{P}$ since $e v_{E_{B}}$ is transversal to $\Delta_{E_{B}}$ for every labeled forest. Hence, $\widetilde{\mathcal{M}}_{\mathcal{S}_{P}}^{* *}\left(P, \mathcal{J}_{P}^{r}\right)$ is a Banach manifold.

The rest of the proof is verbatim the same as in the non-fibered situation and we omit it.

Proof of (I). First, by a standard argument ([8] Lemme 2.3.2, which is a simple adaptation of Proposition 3.2.1 in [17] to the fibered case) involving the use of both the connection induced by $\tau_{H}$ and the $\mathrm{L}-\mathrm{C}$ connection on $T B$, the sets $\mathcal{E}_{P}^{p, r}\left(\beta_{P}\right)$ and $\mathcal{E}_{B}^{p, r}\left(\beta_{B}\right)$ are locally trivial $C^{r-1}$ Banach fibrations over $\mathcal{B}_{P}^{1, p}\left(\beta_{P}, \mathcal{J}_{P}^{r}\right)$ and $\mathcal{B}_{B}^{1, p}\left(\beta_{B}, \mathcal{J}_{B}^{r}\right)$, which can be locally trivialized compatibly with $\mathfrak{p}$. Let $s_{P}$ and $s_{B}$ be the corresponding Cauchy-Riemann sections. The linearization $\widetilde{D}_{\left(u_{B}, J_{B}\right)}^{B}$ of $s_{B}$ at $\left(u_{B}, J_{B}\right)$ is given by (4.2), while the linearization $\widetilde{D}_{\left(u, J_{P}\right)}^{P}$ of $s_{P}$ at $\left(u, J_{P}\right)$ is given by:

$$
\begin{aligned}
& \widetilde{D}_{\left(u, J_{P}\right)}^{P}: \bigoplus_{v \in V} \mathcal{X}_{P, u_{v}}^{1, p} \oplus T_{J_{P}} \mathcal{J}_{P} \rightarrow \bigoplus_{v \in V} \mathcal{E}_{P, u_{v}}^{p}\left(J_{P}\right) \\
&\left(\xi, Y^{v}, Y, f\right) \mapsto D_{u} \xi+\frac{1}{2}\left(Y . d \pi(u) \cdot j_{0}\right)^{h} \\
& \\
&+\frac{1}{2} Y^{v} \cdot(d u)^{v} \cdot j_{0}+X_{f(d u)}^{0,1} .
\end{aligned}
$$

Let $\overline{\mathfrak{p}}$ be the fibration map corresponding to the projection $\mathfrak{p}$ :

$$
\overline{\mathfrak{p}}: \mathcal{E}^{p, r}\left(\beta_{P}\right) \longrightarrow \mathcal{E}_{B}^{p, r}\left(\beta_{B}\right), \quad\left(\eta, J_{P}\right) \mapsto\left(d \pi(\eta), J_{B}\right) .
$$


By definition, $\overline{\mathfrak{p}} \circ s_{P}=s_{B} \circ \mathfrak{p}$. Furthermore, from Lemma 3.2 and since $X_{f(d u)}^{0,1}$ and $Y^{v} \cdot(d u)^{v} \cdot j_{0}$ are vertically valued, we deduce that:

$$
d \overline{\mathfrak{p}} \circ \widetilde{D}_{\left(u, J_{P}\right)}^{P}=\widetilde{D}_{\left(\pi(u), J_{B}\right)}^{B} \circ d \mathfrak{p} .
$$

The maps $d \mathfrak{p}$ and $d \overline{\mathfrak{p}}$ being both surjective, the pair $(\mathfrak{p}, \overline{\mathfrak{p}})$ defines a submersion of Fredholm systems, and we end up having the exact sequence:

$0 \rightarrow \operatorname{ker} \widetilde{D}^{v} \rightarrow \operatorname{ker} \widetilde{D}^{P} \rightarrow \operatorname{ker} \widetilde{D}^{B} \rightarrow \operatorname{coker} \widetilde{D}^{v} \rightarrow \operatorname{coker} \widetilde{D}^{P} \rightarrow \operatorname{coker} \widetilde{D}^{B} \rightarrow 0$,

where $\widetilde{D}^{v}$ denotes the vertical operator associated to $\widetilde{D}^{P}$.

To prove the claim, we show that the pair $(\mathfrak{p}, \overline{\mathfrak{p}})$ defines a splitting when we restrict $s_{P}$ to $\widetilde{\mathcal{M}^{* *}}\left(P, \beta_{P}, \mathcal{J}_{P}^{r}\right)$ and $s_{B}$ to $\widetilde{\mathcal{M}}^{*}\left(B, \beta_{B}, \mathcal{J}_{B}^{r}\right)$. It is enough to prove this when the tree structure of $\mathcal{S}_{P}$ is preserved under $S_{\pi_{*}}$. Let $V_{0}$ denote the subset of $V$ on which $\beta_{B}^{\prime}$ vanishes, and denote by $V_{+}$its complement in $V$. Since $\widetilde{D}^{B}$ is onto for every $\left(u_{B}, J_{B}\right) \in \widetilde{\mathcal{M}}^{*}\left(B, \beta_{B}, \mathcal{J}_{B}^{r}\right)$, it suffices to show that the vertical operator $\widetilde{D}^{v}$ is surjective at every points of $\widetilde{\mathcal{M}}^{* *}\left(P, \beta_{P}, \mathcal{J}_{P}^{r}\right)$. Note that $\widetilde{D}^{v}$ is closed and suppose it is not dense. Then, by Hahn-Banach we would have a non-zero element:

$$
\left\{\eta_{v}\right\}_{v \in V} \in \bigoplus_{v \in V} L^{q}\left(\Lambda^{0,1}\left(S^{2}, u_{v}^{*} T P^{v}\right)\right), \quad \frac{1}{p}+\frac{1}{q}=1,
$$

such that each $\eta_{v}$ is of class $W^{1, p}$, is in the cokernel of $D_{u_{v}}^{v}$, and verifies:

$$
\begin{aligned}
0= & \int_{S^{2}}\left(\sum_{v \in V_{+}}\left\langle\frac{1}{2} Y^{v} \cdot\left(d u_{v}\right)^{v} \cdot j_{0}+X_{f\left(d u_{v}\right)}^{0,1}, \eta_{v}\right\rangle\right. \\
& \left.+\sum_{v \in V_{0}}\left\langle\frac{1}{2} Y^{v} \cdot\left(d u_{v}\right)^{v} \cdot j_{0}, \eta_{v}\right\rangle\right) d \operatorname{vol}_{S^{2}} .
\end{aligned}
$$

Next, we show that we can find $Y^{v}$ and $f$ such that all the components in the sum must be strictly positive unless all the $\eta_{v}$ are identically zero. Let $Z\left(u_{v}\right)$ denote the set of non-injective points of $u_{v}$ and consider the subset in $S^{2}$ :

$$
X\left(u_{v}\right):=Z\left(u_{v}\right) \cup \bigcup_{v^{\prime} \in V_{+}, v^{\prime} \neq v} u_{v}^{-1}\left(u_{v^{\prime}}\left(S^{2}\right)\right) \cup \bigcup_{v^{\prime} \in V_{0}, v^{\prime} \neq v} u_{v}^{-1}\left(u_{v^{\prime}}\left(S^{2}\right)\right) .
$$

Since we consider simple maps, the complement of this set is open dense in $S^{2}$. Let $x_{v}$ be a point of the complement of $X\left(u_{v}\right)$. Then, there is a neighborhood $\mathcal{V}$ of $x_{v}$, which is embedded via $u_{v}$ into a neighborhood $\mathcal{U}_{v}$ of $u\left(x_{v}\right)$ in $P$ and which does not intersect the image of any other $u_{v^{\prime}}$. Now assume $v \in V_{+}$. From transitivity of the action of Hamiltonian diffeomorphisms on 
the manifold, we can find a function $f \in T_{H} \mathcal{H}=\mathcal{H}$ supported in $\mathcal{U}_{v}$ such that:

$$
\int_{S^{2}}\left\langle X_{f\left(d u_{v}\right)}^{0,1}, \eta_{v}\right\rangle d \operatorname{vol}_{S^{2}}>0
$$

When $v$ 's in $V_{0}$, we can also find an element $Y^{v} \in T_{J} \mathcal{J}^{\text {vert }}$ supported in $\mathcal{U}_{v}$ and such that

$$
\int_{S^{2}}\left\langle\frac{1}{2} Y^{v} \cdot\left(d u_{v}\right)^{v} \cdot j_{0}, \eta_{i}\right\rangle d \operatorname{vol}_{S^{2}}>0 .
$$

The neighborhoods $\mathcal{U}_{v}$ can be chosen small enough so that they are pairwise non-intersecting. Set $\left.Y^{v}\right|_{u_{v}}=0$ for $v \in V_{+}$and $\left.f\right|_{u_{v}}=0$ for $v \in V_{0}$. Then $Y^{v}$ and $f$ are well-defined on the whole manifold $P$, which implies that all the $\eta_{v}$ 's are vanishing. This ends the proof of the first claim.

Proof of (II). Assume $e v_{E}$ is transversal to $\Delta_{E}$ when $\mathcal{S}_{P}$ is any labeled forest. Furthermore, suppose that the graph structure of $\mathcal{S}_{P}$ is preserved under $S_{\pi_{*}}$. This is enough since we can always place ourselves in this situation by adding marked points in the fiber components so that they are all equipped with at least three special points. This procedure does not alter the transversality for $e v_{E}$ as the latter does not depend on the infinitesimal movement of the marked points. Let $\mathcal{S}_{P}^{+}$be the stable stratum data resulting from adding the marked points and consider the map

$$
\operatorname{For}^{P}: \widetilde{\mathcal{M}}_{\mathcal{S}_{P}^{+*}}^{+}\left(P, \mathcal{J}_{P}\right) \rightarrow \widetilde{\mathcal{M}}_{\mathcal{S}_{P}}^{* *}\left(P, \mathcal{J}_{P}\right)
$$

that forgets the $k$ added marked points together with stabilizing the resulting map. Define For $^{B}$ in a similar way. Then:

$$
\operatorname{For}^{B} \circ \widetilde{\pi}_{\mathcal{S}_{P}}=\tilde{\pi}_{\mathcal{S}_{P}} \circ \text { For }^{P} .
$$

Clearly $\operatorname{For}^{P}$ is a submersion. It is not hard to see that $\operatorname{For}^{B}$ is also a submersion. Moreover, using an adaptation to the fibered case of Lemma 3.4.7 in [17], one can show that $\widetilde{\pi}_{\mathcal{S}_{P}}$ is also submersion [8].

Proof of (III). The proof proceeds by induction on the number of edges of the labeled forests $\mathcal{S}_{P}$. When the forest has no edge the assertion is vacuous. Suppose it is true for forests with at most $N$ edges and suppose $\mathcal{S}_{P}$ is a forest with $N+1$ edges. Pick any edge given by the pair $\left(y_{v v^{\prime}}, y_{v^{\prime} v}\right)$, cut it out and replace it by the two new marked points $y_{v v^{\prime}}$ and $y_{v^{\prime} v}$. This procedure gives a new forest $\mathcal{S}_{P}^{\prime}$ with two more tails, which satisfies the induction hypothesis and such that the sets $I\left(\mathcal{S}_{P}\right)$ and $I\left(\mathcal{S}_{P}^{\prime}\right)$ coincide. Let $E^{\prime}$ denote the set of edges of $\mathcal{S}_{P}^{\prime}$. Then $e v_{E^{\prime}}$ is transversal to $\Delta_{E^{\prime}}$ so that $\widetilde{\mathcal{M}}_{\mathcal{S}_{P}^{\prime}}^{*}\left(P, \mathcal{J}_{P}^{r}\right)$ is a Banach manifold. Consider the evaluation

$$
e v_{v v^{\prime}}: \widetilde{\mathcal{M}}_{\mathcal{S}_{P}^{\prime}}^{* *}\left(P, \mathcal{J}_{P}^{r}\right) \rightarrow P \times P, \quad\left(u, \mathbf{y}, \mathbf{x}, J_{P}\right) \mapsto\left(u_{v}\left(y_{v v^{\prime}}\right), u_{v^{\prime}}\left(y_{v^{\prime} v}\right)\right) .
$$

We prove that $e v_{v v^{\prime}}$ is transversal to the diagonal $\Delta_{P} \subset P \times P$. Assume that $\pi$ preserves the tree structure of $\mathcal{S}_{P}^{\prime}$ (if not we can add marked points). Let $e v_{v v^{\prime}}^{B}$ be the analog of $e v_{v v^{\prime}}$, but in the case of the base $B$. It is known 
that $e v_{v v^{\prime}}^{B}$ is transversal to $\Delta_{B} \subset B \times B$ at every point of $\widetilde{\mathcal{M}}_{S_{\pi_{*}}\left(\mathcal{S}_{P}^{\prime}\right)}\left(B, \mathcal{J}_{B}^{r}\right)$ ([17], Proposition 6.2.8). Furthermore,

$$
e v_{v v^{\prime}}^{B} \circ \widetilde{\pi}_{\mathcal{S}_{P}^{\prime}}=(\pi \times \pi) \circ e v_{v v^{\prime}},
$$

Since both $\pi \times \pi$ and $\tilde{\pi}$ are submersions it suffices to check that:

$$
\forall \widetilde{u} \in \widetilde{\mathcal{M}}_{\mathcal{S}_{P}^{\prime}}^{* *}\left(P, \mathcal{J}_{P}\right), \quad \text { coker }\left.\operatorname{dev}_{v v^{\prime}}(\widetilde{u})\right|_{\operatorname{ker} d \widetilde{\pi}(\widetilde{u})}=0 .
$$

By the symmetry arising from quotienting $T F \oplus T F$ by $T \Delta_{\mathrm{F}}$, it suffices to show that the restriction of $\operatorname{dev}_{v v^{\prime}}(\widetilde{u})$ to $V$ surjects onto $T_{u_{v}\left(y_{v v^{\prime}}\right)} F \times\{0\}$. But:

$$
W=\left\{\left(\left\{\xi_{v}\right\}, 0,0,0, Y^{v}, f\right) \in T_{\widetilde{u}} \widetilde{\mathcal{M}}_{\mathcal{S}_{P}^{\prime}}^{* *}\left(P, \mathcal{J}_{P}^{r}\right) \mid \xi_{v} \in W^{1, p}\left(u_{v}^{*} T P^{v}\right), \forall v \in V\right\} .
$$

Hence, by definition of $e v_{v v^{\prime}}$ :

$$
\operatorname{dev}_{v v^{\prime}}(\widetilde{u})\left(\left\{\xi_{v}\right\}, 0,0,0, Y^{v}, f\right)=\left(\xi_{v}\left(y_{v v^{\prime}}\right), \xi_{v^{\prime}}\left(y_{v^{\prime} v}\right)\right) .
$$

Now let $(v, 0) \in T_{u_{v}\left(y_{v v^{\prime}}\right)} F \times\{0\}$ and suppose the $i$ component is not ghost, i.e., is not constant. Then choose any $\xi_{v} \in \mathcal{X}_{P, u_{v}}^{1, p, v}$ such that $\xi_{v}\left(y_{v v^{\prime}}\right)=v$. Adapting Lemma 3.4.7 in [17] to the present situation, we can find $Y^{v}$ or $f$ supported in a small enough neighborhood in $P$ (such that it does not intersect the image of any other component) and a vector field $\zeta \in$ $W^{1, p}\left(u_{v}^{*} T P^{v}\right)$ such that: $\zeta\left(y_{v v^{\prime}}\right)=0$ for $v E v^{\prime}$, and $\left(\xi_{v}-\zeta, 0, Y^{v}, 0\right)$ or $\left(\xi_{v}-\right.$ $\zeta, 0,0, f)$ lies in $\operatorname{ker} \widetilde{D}_{\left(u_{v}, J_{P}\right)}$. Then set

$$
\xi_{v^{\prime}}=0 \quad \forall v^{\prime} \neq v .
$$

If $u_{v}$ is ghost, then consider $V_{\mathrm{gh}}(v)$ the vertices of the largest subtree in $\mathcal{S}_{P}^{\prime}$ containing $v \in V$ and consisting only of ghost components. For all $k \in V_{\mathrm{gh}}(v)$ we must have $\xi_{v}=w$. Consider now all the elements $k \in V \backslash V_{\mathrm{gh}}(v)$ such that there exists $v^{\prime} \in V_{\mathrm{gh}}(v)$ for which $k E v^{\prime}$ and write this set as $K$. All these components have a point in commun in the image of the stable map, namely $u_{v}\left(\Sigma_{v}\right)=u_{v}\left(y_{v v^{\prime}}\right)$. Then, for every $m \in K$ choose any $\xi_{m} \in \mathcal{X}_{P, u_{m}}^{1, p, v}$ such that $\xi_{m}\left(y_{m v^{\prime}}\right)=w$. Applying the argument used in the non-ghost case to all the components indexed by $K$ we find vertically valued vector fields $\left\{\zeta_{m}\right\}_{m \in K}$ such that

$$
\zeta\left(y_{m v^{\prime}}\right)=0 \text { when } m E j, m \in K \text { and } v^{\prime} \in V_{\mathrm{gh}}(v),
$$

and such that, for all $m \in K$, either $\left(\xi_{m}-\zeta, 0, Y^{v}, 0\right)$ or $\left(\xi_{m}-\zeta, 0,0, f\right)$ lies in the kernel of $\widetilde{D}_{\left(u_{m}, J_{P}\right)}$. Finally, set $\xi_{v^{\prime}} \equiv 0$ for every component not indexed by $K \sqcup V_{\text {gh }}(v)$.

Remark 4.1. In the above proof it is essential that we allow the connection to vary. In particular, such perturbations enable us to avoid horizontal $J_{P^{-}}$ holomorphic maps, i.e., maps $u$ such that $\operatorname{Im}(d u) \subset$ Hor for which the index of $D_{u}^{v}$ is negative (see [17], Remark 8.3.2.). 
4.2.3. Cobordisms. We end this section by stating the invariance of the moduli spaces under changes of the regular structures. Let $\mathcal{S}_{P}$ be a stable stratum data and let $\mathcal{S}_{B}$ be its projection. Given two regular structures $J_{P}^{0}$ and $J_{P}^{1}$ in $\mathcal{J}_{P \text {,reg }}\left(\mathcal{S}_{P}\right)$, we designate by $\mathcal{J}_{P}\left(J_{P}^{0}, J_{P}^{1}\right)$ the set of paths $\left\{J_{P}^{s}\right\}$ in $\mathcal{J}_{P}, s \in[0,1]$, with endpoints $J_{P}^{0}$ and $J_{P}^{1}$. Similarly define $\mathcal{J}_{B}\left(J_{B}^{0}, J_{B}^{1}\right)$ for pairs $J_{B}^{0}$ and $J_{B}^{1}$ in $\mathcal{J}_{B \text {,reg }}\left(\mathcal{S}_{B}\right)$. For elements $\gamma \in \mathcal{J}_{P}\left(J_{P}^{0}, J_{P}^{1}\right)$ and $\gamma_{B} \in$ $\mathcal{J}_{B}\left(J_{B}^{0}, J_{B}^{1}\right)$ we set:

$\widetilde{\mathcal{W}}_{\mathcal{S}_{P}}^{* *}\left(P,\left\{J_{P}^{s}\right\}\right):=\gamma^{*} \widetilde{\mathcal{M}}_{\mathcal{S}_{P}}^{* *}\left(P, \mathcal{J}_{P}\right) \quad$ and $\quad \widetilde{\mathcal{W}}_{\mathcal{S}_{B}}^{*}\left(B,\left\{J_{B}^{s}\right\}\right):=\gamma_{B}^{*} \widetilde{\mathcal{M}}_{\mathcal{S}_{B}}^{*}\left(B, \mathcal{J}_{B}\right)$.

It is not hard to see that if $\gamma$ is transversal to $p^{\mathcal{J}_{P}}$, and respectively $\gamma_{B}$ is transversal to $p^{\mathcal{J}_{B}}$, the quotient under $G_{\mathcal{S}_{P}}$ and $G_{\mathcal{S}_{B}}$ of the above pullbacks are then oriented manifolds with boundaries and with dimensions:

$$
\operatorname{dim}\left(\mathcal{M}_{\mathcal{S}_{P}}^{* *}\left(P, J_{P}^{0}\right)\right)+1 \quad \text { and } \operatorname{dim}\left(\mathcal{M}_{\mathcal{S}_{B}}^{* *}\left(B, J_{B}^{0}\right)\right)+1 .
$$

In such case, we say that the paths are regular and denote by

$$
\mathcal{J}_{P, \text { reg }}\left(\mathcal{S}_{P}, J_{P}^{0}, J_{P}^{1}\right) \quad \text { and } \quad \mathcal{J}_{B, \text { reg }}\left(\mathcal{S}_{B}, J_{B}^{0}, J_{B}^{1}\right)
$$

the set of such regular paths. Note that a regular path $\gamma$ in $\mathcal{J}_{P}$ projects to a regular path $\gamma_{B}:=p_{1}(\gamma)$ in $\mathcal{J}_{B}$.

For fixed $J_{B} \in \mathcal{J}_{B, \text { reg }}\left(\mathcal{S}_{B}\right)$ and $u_{B} \in \widetilde{\mathcal{M}}_{\mathcal{S}_{B}}^{*}\left(B, J_{B}\right)$, and for pairs $(J, H)^{0}$ and $(J, H)^{1}$ in $\mathcal{J H}_{\text {reg }}\left(u_{B}, J_{B}, \mathcal{S}_{P}\right)$, let $\mathcal{J H}\left((J, H)^{0},(J, H)^{1}\right)$ denote the set of paths in $\mathcal{J}^{V} \times \mathcal{H}$ with endpoints $(J, H)^{0}$ and $(J, H)^{1}$. For any such path $\gamma$, set

$$
\widetilde{\mathcal{W}}_{\mathcal{S}_{P}}^{* *}\left(\pi^{-1}\left(u_{B}\right), J_{B},\left\{(J, H)^{s}\right\}\right):=\gamma^{*}(\widetilde{\pi})^{-1}\left(u_{B}, J_{B}\right) .
$$

If $\gamma$ is transversal to the restriction of $p_{23} \circ p^{\mathcal{J}_{P}}$ to the fiber $(\widetilde{\pi})^{-1}\left(u_{B}, J_{B}\right)$, then the quotient under $G_{\mathcal{S}_{P}}$ of the corresponding pullback is an oriented manifold with boundary, of dimension

$$
\operatorname{dim}\left(\bar{\pi}^{-1}\left(u_{B}\right) \cap \mathcal{M}^{* *}\left(P, J_{P}^{0}\right)\right)+1 .
$$

In such case, we say that $\gamma$ is regular and the set of regular paths is denoted by $\mathcal{J H}_{\text {reg }}\left(u_{B}, J_{B}, \mathcal{S}_{P},(J, H)^{0},(J, H)^{1}\right)$.

Proposition 4.1. The sets $\mathcal{J}_{P, \text { reg }}\left(\mathcal{S}_{P}, J_{P}^{0}, J_{P}^{1}\right), \mathcal{J}_{B, \text { reg }}\left(\mathcal{S}_{B}, J_{B}^{0}, J_{B}^{1}\right)$ and $\mathcal{J H}_{\text {reg }}\left(u_{B}, J_{B}, \mathcal{S}_{P},(J, H)^{0},(J, H)^{1}\right)$ are of second category.

\section{The product formula}

In this section we establish the product formula. Before doing so, we recall the definition of GW invariant for a semi-positive symplectic manifold $(X, \omega)$. For a detailed exposition of the following standard facts, we refer 
to $[\mathbf{1 7}]$ or $[\mathbf{2 1}]$. Let $A \in H_{2}(X, \mathbb{Z})$, and consider the $l$-pointed evaluation map:

$$
e v_{l, J}^{X}: \mathcal{M}_{0, l}^{*}(X, A, J) \rightarrow X^{l}, \quad\left(u, x_{1}, \ldots, x_{l}\right) \mapsto\left(u\left(x_{1}\right), \ldots, u\left(x_{l}\right)\right) .
$$

This defines a $\operatorname{dim}\left(\mathcal{M}_{0, l}^{*}(X, A, J)\right)$-pseudo-cycle of $X^{l}$ for every $J \in \mathcal{J}_{X \text {,reg }} \subset$ $\mathcal{J}_{X}$ where

$$
\mathcal{J}_{X, \text { reg }}:=\bigcap_{\mathcal{S} \in \mathcal{D}_{0, l}^{A}} \mathcal{J}_{X, \mathrm{reg}}(\mathcal{S}) .
$$

Note that $\mathcal{J}_{X \text {,reg }}$ is of second category since $\mathcal{D}_{0, l}^{A}$ is finite. Moreover, $\mathcal{J}_{X \text {,reg }}$ depends on $\omega$.

We recall that a d-dimensional pseudo-cycle in a manifold $X$ is a pair $(M, f)$ where $M$ is an oriented manifold of dimension $d$ and $f: M \rightarrow X$ is a smooth map such that the closure $\overline{f(M)}$ is compact and such that its omegalimit $\Omega_{f}$ is of codimension at least 2 in $X$. Given classes $c_{1}^{X}, \ldots, c_{l}^{X} \in H_{*}(X)$, it is possible to represent them by pseudo-cycles $\left(M_{1}, f_{1}\right), \ldots,\left(M_{l}, f_{l}\right)$ in $X$, of respective $\operatorname{dimensions} \operatorname{dim} M_{i}:=\operatorname{deg}\left(c_{i}^{X}\right)$. We can further assume that these cycles are in general position and such that $e v_{l, J}^{X}$ is strongly transverse to the product cycle

$$
\mathcal{C}:=\left(M_{1}, f_{1}\right) \times \cdots \times\left(M_{l}, f_{l}\right) .
$$

Then, the corresponding $G W$ invariant is the algebraic number of isolated points in the preimage of $\mathcal{C}$ under $e v_{l, J}^{X}$

$$
\left\langle c_{1}^{X}, \ldots, c_{l}^{X}\right\rangle_{0, l, \sigma}^{X, J}:=e v_{l, J}^{X} \cdot \mathcal{C}
$$

which is set to be 0 unless:

$$
2 n(1-l)+2 c_{1}^{T X}(\sigma)+2 l-6+\sum_{i=0}^{l} \operatorname{deg}\left(c_{i}^{X}\right)=0 .
$$

This number only depends on the bordism class of the pseudo-cycles involved. In particular, it does not depend on the regular almost complex structure, which we will drop from the notations.

Now, let $\pi: P \rightarrow B$ be a Hamiltonian fibration with coupling form $\tau$, and let $\iota_{\mathrm{F}}^{P}$ denote the inclusion of $F$ in $P$. Consider $\sigma \in H_{2}(P, \mathbb{Z})$ with $\sigma_{B}=\pi_{*} \sigma \neq 0$. Let $\left(u_{B}, \mathbf{x}\right)$ be an element of $\mathcal{M}_{0, l}^{*}\left(B, \sigma_{B}, J_{B}\right)$. According to diagram (evd) in the Introduction, the product formula is obtained by considering the (respective) intersections of $e v_{\left(u_{B}, \mathbf{x}\right)}, e v_{l, J_{B}}^{B}$, and $e v_{l, J_{P}}^{P}$, with the product pseudo-cycles:

$$
\left(\mathcal{C}^{F}, f^{F}\right):=\prod_{i=1}^{l}\left(M_{i}^{F}, f_{i}^{F}\right), \quad\left(\mathcal{C}^{B}, f^{B}\right):=\prod_{i=1}^{l}\left(M_{i}^{B}, f_{i}^{B}\right)
$$


and

$$
\left(\mathcal{C}^{P}, f^{P}\right):=\prod_{i=1}^{l}\left(M_{i}^{P}, f_{i}^{P}\right),
$$

where, $\left(M_{i}^{F}, f_{i}^{F}\right),\left(M_{i}^{B}, f_{i}^{B}\right)$ and $\left(M_{i}^{P}, f_{i}^{P}\right)$, respectively represent torsion free homology classes, $c_{i}^{F}, c_{i}^{B}$ and $c_{i}^{P}$, verifying condition $(\star)$. In particular,

$$
\begin{cases}\left(M_{i}^{B}, f_{i}^{B}\right)=\left(p t, f_{i}^{B}\right), & \text { for } i=1, \ldots, m, \\ \left(M_{i}^{F}, f_{i}^{F}\right)=\left(F, i d_{\mathrm{F}}\right), & \text { for } i=m+1, \ldots, l .\end{cases}
$$

Furthermore, a $d$-dimensional pseudo-cycle $(M, f)$ in the fiber $F$ of $P$ defines a $d$-dimensional pseudo-cycle $\left(M, \iota_{\mathrm{F}}^{P} \circ f\right)$ in the total space. Similarly, any $d$-dimensional pseudo-cycle $(M, f)$ in $B$ defines a $d+\operatorname{dim} F$ pseudo-cycle $\left(f^{*} P, \bar{f}\right)$ in $P$, where $\bar{f}$ stands for the bundle map associated to $f$. These operations actually preserve the bordism classes. We conclude that:

$$
\left(M_{i}^{P}, f_{i}^{P}\right)= \begin{cases}\left(M_{i}^{F}, \iota_{\mathrm{F}}^{P}\left(f_{i}^{F}\right)\right), & \text { if } i=1, \ldots, m, \\ \left(\left(f_{i}^{B}\right)^{*} P, \bar{f}_{i}^{B}\right), & \text { otherwise. }\end{cases}
$$

Regarding orientations of the product pseudo-cycles, the exact sequence,

$$
0 \longrightarrow d f^{F}\left(T \mathcal{C}_{\mathrm{F}}\right) \stackrel{\left(\iota_{\mathrm{F}}^{P}\right)^{l}}{\longrightarrow} d f^{P}\left(T \mathcal{C}_{P}\right) \stackrel{\pi^{l}}{\longrightarrow} d f^{B}\left(T \mathcal{C}_{B}\right) \longrightarrow 0
$$

gives:

$$
\operatorname{det} d f^{P}\left(T \mathcal{C}_{P}\right) \cong \operatorname{det} d f^{B}\left(T \mathcal{C}_{B}\right) \otimes \operatorname{det} d f^{F}\left(T \mathcal{C}_{\mathrm{F}}\right) .
$$

Therefore, if we choose the cycles $\left(\mathcal{C}_{B}, f^{B}\right)$ and $\left(\mathcal{C}_{\mathrm{F}}, f^{F}\right)$ to be positively oriented the cycle $\left(\mathcal{C}_{P}, f^{P}\right)$ must also be positively oriented. Now, assume the evaluations are pseudo-cycles and that strong transversality with the product cycles is achieved. Then $\left(e v_{l}^{B}\right)^{-1}\left(f^{B}\right)$ is a finite set $\left\{\left(u_{B, \alpha}, \mathbf{x}_{\alpha}\right)\right\}_{\alpha \in A}$ of isolated simple $l$-pointed $J_{B}$-holomorphic maps. For each $\alpha$ let $\iota_{F, \alpha}^{P}$ denote the embedding of $F^{l}$ into $F_{u_{B, \alpha}\left(\mathbf{x}_{\alpha}\right)}^{l}$. Also, in order to simplify notations set

$$
e v_{\alpha}:=e v_{\left(u_{B, \alpha}, \mathbf{x}_{\alpha}\right)}, \quad f_{\alpha}^{F}:=\iota_{F, \alpha}^{P} \circ f^{F},
$$

and write:

$$
n_{\alpha}:=e v_{\alpha} \cdot f_{\alpha}^{F}, \quad n_{B}:=e v_{l}^{B} \cdot f^{B} \quad \text { and } \quad n_{P}:=e v_{l}^{P} \cdot f^{P} .
$$

It follows easily from a dimensional argument that $n_{P}$ is necessarily zero if and only if either $n_{\alpha}$ or $n_{B}$ is necessarily zero. In the notations above the product formula now reads:

$$
\forall \alpha \in A, \quad n_{P}=n_{\alpha} n_{B} .
$$

We will prove this relation, and then prove the Corollary in the last subsection, Before doing so, we make sure that the evaluation maps are simultaneously pseudo-cycles that remain in the same bordism class under change 
of regularizing almost complex structure. This will give meaning to all the numbers in $(\mathbf{P F})$.

5.1. Evaluation maps as pseudo-cycles. We begin by fixing some notations. Define $\mathcal{J}_{B \text {,reg }} \subset \mathcal{J}_{B}$ and $\mathcal{J}_{P \text {,reg }} \subset \mathcal{J}_{P}$ as in (5.1). These sets are of second category. For $J_{B} \in \mathcal{J}_{B \text {,reg }}$ and $u_{B} \in \mathcal{M}^{*}\left(B, \sigma_{B}, J_{B}\right)$, we set

$$
\mathcal{J} \mathcal{H}_{\text {reg }}\left(u_{B}, J_{B}\right):=\bigcap_{\left\{\mathcal{S}_{P} \mid S_{\pi_{*}}\left(\mathcal{S}_{P}\right)=\mathcal{S}_{B}^{\text {top }}\right\}} \mathcal{J} \mathcal{H}_{\text {reg }}\left(u_{B}, J_{B}, \mathcal{S}_{P}\right),
$$

where $\mathcal{S}_{B}^{\text {top }}$ denotes the top stable stratum data for $\overline{\mathcal{M}}\left(B, \sigma_{B}, J_{B}\right)$, i.e., the stratum having only one vertex as a tree structure. By Theorem B', this set is also of second category. Furthermore, for fixed $J_{B}$ we say that the class $\sigma_{B} \in H_{2}(B, \mathbb{Z})$ only admits irreducible effective decompositions with respect to $J_{B}$ if every stratum $\mathcal{M}_{\mathcal{S}_{B}}\left(B, J_{B}\right)$ is only made of irreducible elements. This condition is in particular realized by primitive classes, e.g., the class of a line in $\mathbb{C P}^{n}$ or the diagonal in $S^{2} \times S^{2}$ with the standard product complex structure. Let $\mathcal{J}_{\text {irr }}\left(\sigma_{B}\right)$ denote the subset of $\mathcal{J}_{B}$ with respect to which $\sigma_{B}$ admits only irreducible effective decompositions. Then $\mathcal{J}_{\text {irr }}\left(\sigma_{B}\right)$ is open in $\mathcal{J}\left(B, \omega_{B}\right)$. Nevertheless, nothing guarantees that it is non-empty. In the theorem below, the restriction to $\mathcal{J}_{\text {irr }}\left(\sigma_{B}\right)$ is essential in order to avoid simple stable maps having a reducible projection. We show that all the evaluation maps in the above diagram are pseudo-cycles.

Theorem 5.1. Assume $(\star \star)$ and that $\mathcal{J}_{\mathrm{irr}}\left(\sigma_{B}\right) \neq \emptyset$. Then:

(i) For every $J_{P} \in \mathcal{J}_{P, \text { reg }}$ with $J_{B} \in \mathcal{J}_{\text {irr }}\left(\sigma_{B}\right)$, the evaluation maps, ev $v_{l, J_{B}}^{B}$ and $e v_{l, J_{P}}^{P}$, are pseudo-cycles. Moreover, changing the regular structure along a regular path induces a bordism between the relevant evaluation maps, as long as the almost complex structure on $B$ varies in a connected component of $\mathcal{J}_{\text {irr }}\left(\sigma_{B}\right)$.

(ii) Fix a regular structure $J_{B}$, and let $\left(u_{B}, \mathbf{x}\right) \in \mathcal{M}_{0, l}^{*}\left(B, J_{B}, \sigma_{B}\right)$. Then, for any element in $\mathcal{J H}_{\text {reg }}\left(u_{B}, J_{B}\right)$, the couple $\left(\bar{\pi}^{-1}\left(u_{B}, \mathbf{x}\right), e v_{\left(u_{B}, \mathbf{x}\right)}\right)$ is a pseudo-cycle that remains in the same bordism class, under change of regularizing pair along regular paths.

Proof. We only show the first statement, the proof of the second being sim-

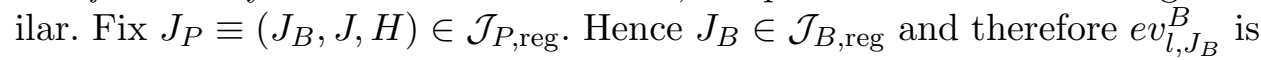
a pseudo-cycle ([17], Theorem 6.6.1). By Lemma 4.3, there are only finitely many stable stratum data $\mathcal{S}_{P}$ representing geometric limits of curves in $\mathcal{M}_{0, l}^{* *}\left(P, \sigma, J_{P}\right)$. Hence, by Gromov's compactness

$$
\Omega_{e v_{l, J_{P}}^{P}} \subset \bigcup_{\mathcal{S}_{P}} e v_{l, J_{P}}^{P}\left(\mathcal{M}_{\mathcal{S}_{P}}^{* *}\left(P, J_{P}\right)\right),
$$


where the union is taken over all reduced stratum data. Let $\beta_{P}^{\text {red }}$ denote the homological labeling associated to a reduction $\mathcal{S}_{P}^{\text {red }}$ of $\mathcal{S}_{P}$. Let $V_{+}^{\text {red }}$ denote the set of $\pi$-stable components in $\mathcal{S}_{P}^{\text {red }}$ and let $V_{0}^{\text {red }}$ denote the set of $\pi$-unstable components in $\mathcal{S}_{P}^{\text {red }}$. Since $\sigma_{B}$ only admits irreducible decompositions, only $\pi$-unstable components of $\mathcal{S}_{P}$ are contracted in the reduction process. Therefore, there exist integers $m_{v}>0$ for all $v \in V_{0}^{\text {red }}$ such that:

$$
\sigma=\sum_{v \in V_{+}^{\text {red }}} \beta_{P}^{\text {red }}(v)+\sum_{v \in V_{0}^{\text {red }}} m_{v} \beta_{P}^{\text {red }}(v)
$$

Condition $(\star \star)$ further implies that $c^{v}\left(\beta_{P}^{\text {red }}(v)\right)>0$ for every $v \in V_{0}^{\text {red }}$, and we conclude that

$$
\operatorname{dim} \mathcal{M}_{\mathcal{S}_{P}^{\text {red }}}^{* *}\left(P, J_{P}\right) \leq \operatorname{dim} \mathcal{M}_{0, l}^{* *}\left(P, \sigma, J_{P}\right)-2,
$$

for every stratum reduction. Hence, $e v_{l, J_{P}}^{P}$ is a pseudo-cycle. The independence statement is shown as follows. Let $J_{P}^{t}$ be a regular path of fibered almost complex structures between regular fibered structures projecting on a path in $\mathcal{J}_{B \text {,reg }}\left(J_{B}^{0}, J_{B}^{1}\right) \cap \mathcal{J}_{\text {irr }}\left(\sigma_{B}\right)$, where $J_{B}^{0}, J_{B}^{1}$ lie in the same connected component of $\mathcal{J}_{\text {irr }}\left(\sigma_{B}\right)$. Then, any Gromov limit of a sequence in $\mathcal{W}_{0, l}^{* *}\left(P, \sigma,\left\{J_{P}^{t}\right\}\right)$ is such that its non-trivial roots are irreducible, while its fiber components may actually be reducible. Arguing exactly as above, the lower strata in $\overline{\mathcal{W}}_{0, l}^{* *}\left(P, \sigma,\left\{J_{P}^{t}\right\}\right)$ have codimension at least 2 in $P$. Thus,

$$
e v_{l,\left\{J_{P}^{t}\right\}}^{P}: \mathcal{W}_{0, l}^{* *}\left(P, \sigma,\left\{J_{P}^{t}\right\}\right) \rightarrow P^{l}
$$

is a pseudo-cycle inducing a bordism between $e v_{l, J_{P}^{0}}^{P}$ and $e v_{l, J_{P}^{1}}^{P}$.

Remark 5.1. Note that in this context we do not need to impose any semipositivity assumption on $B$ due to the specific decomposability hypothesis imposed on $\sigma_{B}$. Note also that if $\sigma_{B}$ is undecomposable, we can drop the restriction on $\mathcal{J}_{\text {irr }}\left(\sigma_{B}\right)$.

5.2. Proof of Theorem A. We begin by proving that all the terms in $(\mathbf{P F})$ are well-defined. From Theorem 5.1 and since $\mathcal{J}_{\text {irr }}\left(\sigma_{B}\right) \neq \emptyset$, the evaluation maps $e v_{l}^{B}$ and $e v_{l}^{P}$ generically define pseudo-cycles. Choose (generically) the cycles $\left(M_{i}^{B}, f_{i}^{B}\right)$ so that $e v_{l}^{B}$ is strongly transverse to $\left(\mathcal{C}_{B}, f^{B}\right)$. Then $\left(e v_{l}^{B}\right)^{-1}\left(f^{B}\right)$ is finite and, as already mentioned, the corresponding GWinvariant $n_{B}$ only depends on the bordism classes of $\left(M_{i}^{B}, f_{i}^{B}\right)$, and on the connected components of $\mathcal{J}_{\text {irr }}\left(\sigma_{B}\right) \cap \mathcal{J}_{B \text {,reg. Thus, }}$

$$
\left(e v_{l}^{B}\right)^{-1}\left(f^{B}\right)=\left\{\left(u_{B, \alpha}, \mathbf{x}_{\alpha}\right) \mid \alpha \in A\right\}
$$

is finite, and for every $\alpha \in A$ the map $e v_{\alpha}$ also defines a pseudo-cycle for generic fiber regularizing pairs. Consequently, $\left(F^{l}, f_{\alpha}^{F}\right)$ is a pseudo-cycle of $F_{u_{B}\left(\mathbf{x}_{\alpha}\right)}^{l}$ for every $\alpha \in A$. Since $A$ is finite, we can furthermore choose the cycles $\left(M_{i}^{F}, f_{i}^{F}\right)$ such that $e v_{\alpha}$ is transversal to $f_{\alpha}^{F}$ for every $\alpha \in A$. This, 
together with the fact that $e v_{l}^{B}$ is transversal to $f^{B}$, implies that $e v_{l}^{P}$ is transversal to $f^{P}$. The independence of $n_{\alpha}$ and $n_{P}$ with respect to the choice of regularizing triple follows from Theorem 5.1.

Next, we prove $(\mathbf{P F})$. Let $C_{\alpha} \subset B$ denote the image of $u_{B, \alpha}$, let $P_{C_{\alpha}}$ be the restriction of $P$ to $C_{\alpha}$, and let $\iota_{\alpha}: P_{C_{\alpha}} \hookrightarrow P$ and $\iota_{\mathrm{F}}^{P_{C_{\alpha}}}: F \hookrightarrow P_{C_{\alpha}}$ denote the natural inclusions. Consider the subset of section classes in $P_{C_{\alpha}}$

$$
B_{\sigma}^{\alpha}=\left\{\sigma^{\prime} \in H_{2}\left(P_{C_{\alpha}}, \mathbb{Z}\right) \mid\left(\iota_{\alpha}\right)_{*} \sigma^{\prime}=\sigma\right\}
$$

Then,

$$
n_{\alpha}=\sum_{\sigma^{\prime} \in B_{\sigma}^{\alpha}}\left\langle\iota_{\mathrm{F}}^{P_{C_{\alpha}}}\left(c_{1}^{F}\right), \ldots, \iota_{\mathrm{F}}^{P_{C_{\alpha}}}\left(c_{l}^{F}\right)\right\rangle_{0, l, \sigma^{\prime}}^{P_{C_{\alpha}}}
$$

Indeed, let $J_{P_{C_{\alpha}}}$ denote the restriction of $J_{P}$ to $P_{C_{\alpha}}$, then $\iota_{\alpha}$ naturally induces an identification:

$$
\bar{\iota}_{\alpha}: \bigsqcup_{\sigma^{\prime} \in B_{\sigma}^{\alpha}} \overline{\mathcal{M}}\left(P_{C_{\alpha}}, J_{P_{C_{\alpha}}}, \sigma^{\prime}\right) \rightarrow \bar{\pi}^{-1}\left(u_{B, \alpha}, \mathbf{x}_{\alpha}\right),
$$

which is an orientation preserving diffeomorphism when restricted to any stratum. Furthermore, by simplicity of $u_{B, \alpha}$ the $l$ marked points are naturally identified to the $2 l$-dimensional manifold $\mathcal{M}_{0, l}^{*}\left(C_{\alpha},\left[C_{\alpha}\right]\right)$. We obtain the following diagram:

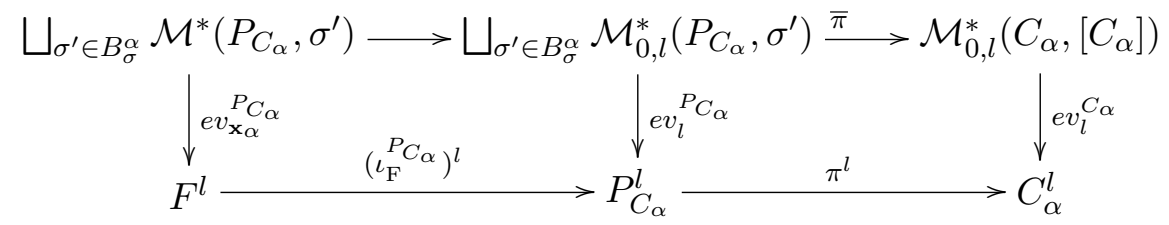

where the complex structures are omitted in order to simplify notations. By definition, $e v_{\mathbf{x}_{\alpha}}^{P_{C_{\alpha}}}$ is the composition of $e v_{\alpha}$ with $\iota_{\alpha}$ hence is a pseudo-cycle for generic fiber regularizing parameters. Using the above diagram we conclude that $e v_{l}^{P_{C_{\alpha}}}$ is generically a pseudo-cycle. Then, equation (5.2) follows since there is only one positively oriented curve in $\mathcal{M}_{0, l}^{*}\left(C_{\alpha},\left[C_{\alpha}\right]\right)$ intersecting transversally $l$ points at the $l$-marked points (giving $\langle p t, \ldots, p t\rangle_{0, l,\left[C_{\alpha}\right]}^{C_{\alpha}}=1$ ).

Now, consider the sign functions $\epsilon_{P}, \epsilon_{B}$, and $\epsilon_{\alpha}$, respectively associated to the curves counted in $n_{P}, n_{B}$ and $n_{\alpha}$. We have to make sure that the signs of the counted curves are given compatibly with $\pi^{l}$, i.e., that:

$$
\forall \alpha \in A, \quad \epsilon_{P}=\epsilon_{B} \times \epsilon_{\alpha} .
$$

However, this is the case since for every fiber regularizing pair we have the exact sequence:

$$
\begin{aligned}
0 & \rightarrow T_{u}\left(\bar{\pi}^{-1}\left(u_{B, \alpha}, \mathbf{x}_{\alpha}\right)\right) \rightarrow T_{\left(\iota_{\alpha}(u), \mathbf{x}_{\alpha}\right)} \mathcal{M}_{0, l}^{* *}(P, \sigma) \\
& \rightarrow T_{\left(u_{B, \alpha}, \mathbf{x}_{\alpha}\right)} \mathcal{M}_{0, l}^{*}\left(B, \sigma_{B}\right) \rightarrow 0 .
\end{aligned}
$$


Consequently:

$$
\begin{aligned}
n_{P} & =\sum_{\alpha \in A} \sum_{\left\{u \in e v_{\alpha}^{-1}\left(f_{\alpha}^{F}\right)\right\}} \epsilon_{P}\left(\iota_{\alpha}(u)\right) \\
& =\sum_{\alpha \in A}\left(\epsilon_{B}\left(u_{B, \alpha}, \mathbf{x}_{\alpha}\right) \sum_{\left\{u \in e v_{\alpha}^{-1}\left(f_{\alpha}^{F}\right)\right\}} \epsilon_{P_{C_{\alpha}}, \mathbf{x}_{\alpha}}(u)\right),
\end{aligned}
$$

which, by Lemma 2.3, coincides with $n_{\alpha_{0}} n_{B}$ for any $\alpha_{0} \in A$.

\section{Gluing and fibration of moduli spaces}

The aim of this section is to show that, under some circumstances, $\pi$ defines a locally trivial differentiable orbi-fibration above the top stratum of $\overline{\mathcal{M}}_{0, l}\left(B, \sigma_{B}, J_{B}\right)$, with $\sigma_{B} \neq 0$. We start with the following simple observation. From the transversality theorem the restriction of $\bar{\pi}_{\mathcal{S}_{P}}$ to $\mathcal{M}^{* *}\left(P, \sigma, J_{P}\right)$ is generically a smooth submersion onto $\mathcal{M}^{*}\left(B, \sigma_{B}, J_{B}\right)$ over countably many points. It is natural to ask if there exists a fibered $J_{P}$ with respect to which the latter map is everywhere regular. For fixed $J_{B}$ and $J$, we say that $H \in \mathcal{H}$ is parametric if $D_{u}^{v}$ is surjective for every $u \in \mathcal{M}^{* *}\left(P, \sigma, J_{P}\right)$. From exactness of (3.4) $\pi$ is a smooth submersion for parametric $H$. As a result, if we assume that $\sigma$ is an undecomposable effective class projecting onto a non-trivial undecomposable class in $B$ and that $H$ is parametric, then $\bar{\pi}_{\mathcal{S}_{P}}$ is a locally trivial fibration (Indeed, since $\sigma$ is undecomposable, $\mathcal{M}^{* *}\left(P, \sigma, J_{P}\right)$ is compact so that $\pi_{\mathcal{S}_{P}}$ is proper, as desired).

Remark 6.1. The set of parametric $H$ 's may be empty. Consider the $\left(\mathbb{C P}^{1}, \omega_{F S}\right)$-fibration,

$$
\pi: E:=\mathbb{P}\left(\mathcal{O}_{\mathbb{C P}^{2}}(-2) \oplus \mathbb{C}\right) \longrightarrow\left(\mathbb{C P}^{2}, \omega_{F S}\right) .
$$

Denote by $L$ the homology class of a line in $\mathbb{C P}^{2}$, and let $L_{0} \in H_{2}(E, \mathbb{Z})$ be the class such that $L=\pi_{*}\left(L_{0}\right)$ and $L_{0} \cap[F]=[p t]$, where $[F]$ stands for the class of a fiber in $E$. If $u$ is a holomorphic curve in $E$ representing $L_{0}$, the fibration $u^{*} T E$ is isomorphic to the direct sum of $\mathcal{O}_{\mathbb{C P}^{1}}(2), \mathcal{O}_{\mathbb{C P}^{1}}(1)$ and $\mathcal{O}_{\mathbb{C P}^{1}}(-2)$. A straightforward computation then shows that the index of the vertical linearized operator must be -2 , hence there are no parametric $H$.

We will make the following assumption on the fibered structure $J_{P}$ throughout this section:

Split Regularity Assumption (SR). For every stable stratum data $\mathcal{S}_{P}$ for pseudo-holomorphic maps in $P$ representing $\sigma$, for every $u \in$ $\widetilde{\mathcal{M}}_{\mathcal{S}_{P}}\left(P, J_{P}\right)$ :

- the operators $D_{u}^{v}$ and $D_{\pi(u)}^{B}$ are surjective; 
- the edge evaluation maps $e v^{B}$ and $e v_{\pi_{\mathcal{S}_{P}}(u)}$ are transversal to the corresponding diagonals.

This implies that for every $u \in \mathcal{M}_{\mathcal{S}_{P}}\left(P, J_{P}\right)$ the operator $D_{u}^{P}$ is surjective, and $e v^{P}$ is transverse to the associated diagonal (see Section 3). By a standard argument the quotient spaces $\mathcal{M}_{\mathcal{S}_{P}}\left(P, J_{P}\right), \mathcal{M}_{\mathcal{S}_{B}}\left(B, J_{B}\right)$, and the fiber of $\bar{\pi}_{\mathcal{S}_{P}}$ over $\bar{\pi}_{\mathcal{S}_{P}}(u)$, are smooth orbifolds. Furthermore, the commutativity $\pi_{*} \circ D_{u}^{P}=D_{\pi_{\mathcal{S}_{P}}(u)}^{B} \circ \pi_{*}$ implies that these orbifold structures can be chosen compatibly with $\bar{\pi}_{\mathcal{S}_{P}}$. We will drop the almost complex structures from the notations since it is understood that we made a choice here.

Under the split regularity assumption, we will prove Theorem C. Namely,

Theorem 6.1. Under hypothesis (SR), the moduli spaces $\overline{\mathcal{M}}_{0, l}(P, \sigma)$ and $\overline{\mathcal{M}}_{0, l}\left(B, \sigma_{B}\right)$ are $C^{1}$-orbifolds, and the maps $\bar{\pi}_{\mathcal{S}_{P}}$ extend to a map

$$
\bar{\pi}: \overline{\mathcal{M}}_{0, l}(P, \sigma) \rightarrow \overline{\mathcal{M}}_{0, l}\left(B, \sigma_{B}\right),
$$

which restricts to a $C^{1}$ locally trivial fibration (of orbifolds) above each strata of $\overline{\mathcal{M}}_{0, l}\left(B, \sigma_{B}\right)$.

The main idea of the proof is to construct gluing maps compatibly with the projections $\bar{\pi}_{\mathcal{S}_{P}}$. We explain this. In general, a gluing map will refer to a map $G l_{\mathcal{S}_{P}}$ with values in $\mathcal{M}_{0, l}(P, \sigma)$ and with domain the complement $\mathcal{L}_{\mathcal{S}_{P}}^{*}$ of the zero-section of an orbibundle of complex vector spaces (the gluing parameters) $\mathcal{L}_{\mathcal{S}_{P}}$ over $\mathcal{M}_{\mathcal{S}_{P}}(P)$. In the fibered context, under hypothesis (SR), we will construct gluing maps $G l_{\mathcal{S}_{P}}: \mathcal{L}_{\mathcal{S}_{P}}^{*} \rightarrow \mathcal{M}_{0, l}(P, \sigma)$ and $G l_{\mathcal{S}_{B}}$ : $\mathcal{L}_{\mathcal{S}_{B}}^{*} \rightarrow \mathcal{M}_{0, l}\left(B, \sigma_{B}\right)$ such that:

$$
\bar{\pi} \circ G l_{\mathcal{S}_{P}}=G l_{\mathcal{S}_{B}} \circ \overline{\bar{\pi}}{\overline{\mathcal{S}_{P}}},
$$

where $\overline{\bar{\pi}} \mathcal{S}_{P}: \mathcal{L}_{\mathcal{S}_{P}} \rightarrow \mathcal{L}_{\mathcal{S}_{B}}$ is an appropriate orbibundle map. More precisely, let $U_{P} \subset \mathcal{M}_{\mathcal{S}_{P}}(P)$ and $U_{B} \subset \mathcal{M}_{\mathcal{S}_{B}}(B)$ be proper open subsets such that $\bar{\pi}_{\mathcal{S}_{P}}\left(U_{P}\right)=U_{B}$. For $\epsilon_{B}$ a positive real, let $\mathcal{L}_{\mathcal{S}_{B}, \epsilon_{B}}$ denote the $\epsilon_{B^{-}}$ neighborhood of the zero-section in $\mathcal{L}_{\mathcal{S}_{B}}$, and let $\mathcal{L}_{\mathcal{S}_{B}, \epsilon_{B}, U_{B}}$ be the restriction of $\mathcal{L}_{\mathcal{S}_{B}, \epsilon_{B}}$ to $U_{B}$. Define $\mathcal{L}_{\mathcal{S}_{P}, \epsilon_{P}, U_{P}}$ similarly. We will prove the following:

Theorem 6.2. For small enough positive constants $\epsilon_{P}$ and $\epsilon_{B}$, there are $\left(C^{1}\right)$-diffeomorphisms:

$$
G l_{\mathcal{S}_{P}}: \mathcal{L}_{\mathcal{S}_{P}, \epsilon_{P}, U_{P}}^{*} \rightarrow \mathcal{M}_{0, l}(P, \sigma), \quad G l_{\mathcal{S}_{B}}: \mathcal{L}_{\mathcal{S}_{B}, \epsilon_{B}, U_{B}}^{*} \rightarrow \mathcal{M}_{0, l}\left(B, \sigma_{B}\right)
$$

such that:

$$
\bar{\pi} \circ G l_{\mathcal{S}_{P}}=G l_{\mathcal{S}_{B}} \circ \overline{\bar{\pi}}{\mathcal{\mathcal { S } _ { P }}} .
$$

The gluing maps in Theorem 6.2 will provide $C^{1}$-differentiable atlases on the moduli spaces involved, giving the desired extension $\bar{\pi}$. The fibration structure statement then follows easily since each $\bar{\pi}_{\mathcal{S}_{P}}$ is a submersion (in the topology induced by the gluing maps). This implies that when restricted 
to a given strata of $\overline{\mathcal{M}}_{0, l}\left(B, \sigma_{B}\right)$, the projection $\bar{\pi}$ is a proper surjective differentiable submersion, hence we have a locally trivial fibration.

This section is divided in three parts. The first two parts deal with the gluing procedure. The first part, Section 6.1 , is expository following Chen and Li's work [3]. In the second part, Section 6.2, we give the proof of Theorem 6.2. The proof is an adaptation to the fibered case, of Chen and Li's work, presenting no major difficulty. Finally, we prove Theorem 6.1 in Section 6.3: namely, we show that gluing maps provide differentiable atlases on the moduli spaces compatibly with the projections $\bar{\pi}_{\mathcal{S}_{P}}$.

6.1. Gluing in the non-fibered case: $\boldsymbol{B}=\boldsymbol{p t}$. We give the gluing procedure in $\overline{\mathcal{M}}_{0, l}(X, A)$ with $(X, \omega)$ a general symplectic manifold. This will be our guideline when considering more general base. We start by gluing in $\mathcal{M}_{0, l}$. We follow standard approaches in the literature such as $[\mathbf{3}, \mathbf{1 7}, \mathbf{2 0}, \mathbf{2 3}]$, among others.

6.1.1. Gluing for nodal curves. Let $\mathcal{S}:=(V, \mathrm{Fl} ; \mathrm{pr}, \varrho)$ be a stable stratum data for $\mathcal{M}_{0, l}$, and let $\mathbf{j} \equiv(\Sigma, \mathbf{j}, \mathbf{x}) \in \mathcal{M}_{\mathcal{S}}$. For $v \in V$, let $\Sigma_{v}$ denote the corresponding (irreducible) component, and for $f \in \mathrm{Fl}$, denote by $z_{f}$ the corresponding special point on $\Sigma_{v}$. By definition each $\Sigma_{v}$ is stable. Then, up to isometry, there exists a unique isometric action of a Fuchsian group $\Gamma$ on the hyperbolic half plane $\mathbb{H}$ with respect to which:

$$
\Sigma_{v} \backslash\left\{z_{f} \mid \operatorname{pr}(f)=v\right\} \cong \mathbb{H} / \Gamma .
$$

The induced metric belongs to the conformal class given by the complex structure on $\Sigma_{v}$. If $D \subset \mathbb{H}$ denotes a Dirichlet region of $\Gamma$, then each $z_{f}$ corresponds to a vertex at infinity, and we can choose the fundamental region $D$ such that $z_{f}$ corresponds to infinity with edges $x \equiv 0$ and $x \equiv 1$. It is well known that given a real $b>1$, the horocycle at $z_{f}$,

$$
\{x+i y \in \mathbb{H} \mid 2 \pi y>b\} /\langle z \mapsto z+1\rangle,
$$

defines a neighborhood of $z_{f}$, which can be identified to a punctured disc $D^{*}\left(e^{1-b}\right) \subset \mathbb{C}$ via the map $z \mapsto e^{2 \pi i z+1}$. We have such neighborhoods for each $z_{f}$, and we denote them $D_{f}^{*}\left(r_{f}\right)$, with small $r_{f}>0$. Now, $D_{f}^{*}\left(r_{f}\right)$ is conformally equivalent to a cylinder with negative end:

$$
\left(-\infty, \ln r_{f}\right] \times \mathbb{R} / 2 \pi \mathbb{Z} \cong D_{f}^{*}\left(r_{f}\right):(s, t) \mapsto e^{s+i t}
$$

or with positive end:

$$
\left[-\ln r_{f},+\infty\right) \times \mathbb{R} / 2 \pi \mathbb{Z} \cong D_{f}^{*}\left(r_{f}\right):(s, t) \mapsto e^{-s-i t} .
$$

Let $e_{f, f^{\prime}}$ be an edge of $\mathcal{S}$ between the vertices $v:=\operatorname{pr}(f)$ and $v^{\prime}:=\operatorname{pr}\left(f^{\prime}\right)$. For a complex number

$$
\rho_{v v^{\prime}}:=e^{-R_{v v^{\prime}}+i \theta_{v v^{\prime}}} \equiv r_{v v^{\prime}} e^{i \theta_{v v^{\prime}}}, \quad \theta \in[0,2 \pi),
$$


such that $\left|\rho_{v v^{\prime}}\right|<\min \left\{r_{f}, r_{f^{\prime}}\right\}$, we can glue the components $\Sigma_{v}$ and $\Sigma_{v^{\prime}}$ at $z_{f}$ and $z_{f^{\prime}}$ as follows. Put positive (resp. negative) cylindrical coordinates on $D_{f}^{*}\left(r_{f}\right)$ (resp. $D_{f^{\prime}}^{*}\left(r_{f^{\prime}}\right)$ and identify the annuli:

$$
\begin{aligned}
{\left[-R_{v v^{\prime}} / 2-1,-R_{v v^{\prime}} / 2+1\right] \times \mathbb{R} / 2 \pi \mathbb{Z} } & \cong\left[R_{v v^{\prime}} / 2-1, R_{v v^{\prime}} / 2+1\right] \times \mathbb{R} / 2 \pi \mathbb{Z}, \\
(s, t) & \mapsto\left(s+R_{v v^{\prime}}, t+\theta_{v v^{\prime}}\right) .
\end{aligned}
$$

This gives the patching procedure between $\mathcal{A}_{f}:=D_{f}^{*}\left(r_{f}\right) \backslash D_{f}^{*}\left(e^{-R_{v v^{\prime}}}\right)$ and $\mathcal{A}_{f^{\prime}}:=D_{f^{\prime}}^{*}\left(r_{f^{\prime}}\right) \backslash D_{f^{\prime}}^{*}\left(e^{-R_{v v^{\prime}}}\right)$. The resulting curve $\Sigma_{\rho_{v v^{\prime}}}\left(f, f^{\prime}\right)$ has a natural complex structure and is the gluing of $\mathbf{j}$ at $e_{f, f^{\prime}}$ with parameter $\rho_{v v^{\prime}}$.

Note that $\rho_{v v^{\prime}}$ can be naturally identified to an element of

$$
\mathbb{C}_{v v^{\prime}}:=T_{y_{v v^{\prime}}} \Sigma_{v} \otimes T_{y_{v^{\prime} v}} \Sigma_{v^{\prime}} \cong \mathbb{C}, \quad v E v^{\prime} .
$$

Denote by $\mathbb{C}_{\mathbf{j}}$ the direct sum of the $\mathbb{C}_{v v^{\prime}}$ over all edges in $\mathcal{S}$, and by $B_{\epsilon}$ the ball of radius $\epsilon$ at the origin of $\mathbb{C}_{\mathbf{j}}$. Then, for small enough $\epsilon>0$, the gluing gives a map:

$$
g l_{\mathbf{j}}: B_{\epsilon} \subset \mathbb{C}_{\mathbf{j}} \rightarrow \overline{\mathcal{M}}_{0, l}, \quad \rho \mapsto \mathbf{j}_{\rho} \equiv \Sigma_{\rho} .
$$

which coincides with the identity map when $\rho \equiv 0$. Note that $g l_{\mathbf{j}}$ is $\operatorname{Aut}(\mathbf{j})$ equivariant (for the linear action of $\operatorname{Aut}(\mathbf{j})$ on $\mathbb{C}_{\mathbf{j}}$ ), and it follows from stability of the curve that $g l_{\mathbf{j}}$ is injective. Taking the union over $\mathcal{M}_{\mathcal{S}}$ of the $\mathbb{C}_{\mathbf{j}}$ actually defines an orbibundle

$$
p_{\mathcal{S}}: \mathcal{L}_{\mathcal{S}} \rightarrow \mathcal{M}_{\mathcal{S}}
$$

Let $\mathcal{L}_{\mathcal{S}, \epsilon}$ denote the restriction to an $\epsilon$ neighborhood of the zero section, and let $\mathcal{L}_{\mathcal{S}}^{*}$ be $\mathcal{L}_{\mathcal{S}}$ with the zero section removed. Given a proper open subset $U \subset \mathcal{M}_{\mathcal{S}}$, there exists $\epsilon>0$, depending on $U$, such that the above gluing map extends to a diffeomorphism:

$$
g l_{\mathcal{S}}: \mathcal{L}_{\mathcal{S}, \epsilon, U}^{*}:=\left.\mathcal{L}_{\mathcal{S}, \epsilon}^{*}\right|_{U} \rightarrow \overline{\mathcal{M}}_{0, l}, \quad(\mathbf{j}, \rho) \mapsto g l_{\mathbf{j}}(\rho) .
$$

More generally, given two stable stratum data such that $\mathcal{S} \prec \mathcal{S}^{\prime}$, there exists a subbundle $\mathcal{L}_{\mathcal{S}, \mathcal{S}^{\prime}}$ of $\mathcal{L}_{\mathcal{S}}$ with fibers identified to $\mathbb{C}^{\left|E^{\prime}\right|-|E|},\left|E^{\prime}\right|$ being the number of edges in $\mathcal{S}^{\prime}$, as well as gluing maps (defined on proper open subsets):

$$
g l_{\mathcal{S}, \mathcal{S}^{\prime}}: \mathcal{L}_{\mathcal{S}, \mathcal{S}^{\prime}, \epsilon}^{*} \rightarrow \overline{\mathcal{M}}_{\mathcal{S}} .
$$

When $\mathcal{S}$ is unstable, one can still define $\mathcal{L}_{\mathcal{S}}$ over $\mathcal{M}_{\mathcal{S}}$, as well as a gluing map $g l_{\mathcal{S}}$, but $g l_{\mathcal{S}}$ is injective and a local diffeomorphism if and only if $\mathcal{S}$ is stable.

6.1.2. Gluing stable components. Let $\mathcal{S}_{X}:=(V, \mathrm{Fl} ; \mathrm{pr}, \varrho)$ be a stable stratum data. Here we assume that the forgetful map $\mathcal{S}:=S_{\pi_{p t_{*}}}\left(\mathcal{S}_{X}\right)$ and $\mathcal{S}_{X}$ have the same tree structure. Then $\bar{\pi}_{p t}^{\mathcal{S}_{X}}$ coincides with the forgettingthe-map map:

$$
\mathcal{F}_{X}: \mathcal{M}_{\mathcal{S}_{X}}(X) \rightarrow \mathcal{M}_{\mathcal{S}}, \quad(u, \mathbf{j}) \mapsto \mathbf{j}
$$


Let $\mathcal{L}_{\mathcal{S}_{X}}$ denote the orbibundle $\mathcal{F}_{X}^{*} \mathcal{L}_{\mathcal{S}}$ over $\mathcal{M}_{\mathcal{S}_{X}}(X)$. In local coordinates, an element in $\mathcal{L}_{\mathcal{S}_{X}}$ is given by a triple $(u, \mathbf{j}, \rho)$. Consider the bundle map:

$$
\overline{\bar{\pi}}_{p t} \mathcal{S}_{X}: \mathcal{L}_{\mathcal{S}_{X}} \rightarrow \mathcal{L}_{\mathcal{S}}, \quad(u, \mathbf{j}, \rho) \mapsto(\mathbf{j}, \rho) .
$$

The gluing consists in constructing, compatibly with $\overline{\bar{\pi}}_{p t} \mathcal{S}_{X}$, a $J$-holomorphic map with domain $g l_{\mathcal{S}}(\mathbf{j}, \rho)$ out of any $(u, \mathbf{j}, \rho)$. More precisely, the following holds:

Theorem 6.3 ([3] Theorem 12.1, [17] Theorem 10.1.2, [20] Theorem 3.34). For every proper subset $U_{X} \subset \mathcal{M}_{\mathcal{S}_{X}}(X)$, there exists a constant, $\epsilon_{X}>0$, and a diffeomorphism:

$$
G l_{\mathcal{S}_{X}}: \mathcal{L}_{\mathcal{S}_{X}, \epsilon_{X}, U_{X}}^{*} \rightarrow \mathcal{M}_{0, l}(X, A), \quad(u, \mathbf{j}, \rho) \mapsto G l_{\mathcal{S}_{X}}(u, \mathbf{j}, \rho),
$$

such that

$$
\bar{\pi}_{p t} \circ G l_{\mathcal{S}_{X}}=g l_{\mathcal{S}} \circ \overline{\bar{\pi}}_{p t} \mathcal{S}_{X} .
$$

Proof. We give a sketched proof of this theorem that serves as a guiding principle when gluing pseudo-holomorphic curves. This is done in several steps.

Step 1: pregluing. Here, we construct an approximatively $J$-holomorphic smooth map out of $(u, \mathbf{j}, \rho) \in \mathcal{L}_{\mathcal{S}_{X}}^{*}$. For $v \in V$, let $u_{v}: \Sigma_{v} \rightarrow X$ denote the corresponding component of $u$. Also, for an edge $e_{f, f^{\prime}}$ of $\mathcal{S}_{X}$ with $\operatorname{pr}(f)=v$ and $\operatorname{pr}\left(f^{\prime}\right)=v^{\prime}$, let $y_{v, v^{\prime}}$ denote the corresponding node on $\Sigma$. Let $\rho_{v v^{\prime}} \in \mathbb{C}^{*}$, defined by (6.1), denote the gluing parameter associated to $y_{v, v^{\prime}}$. Furthermore, let $\beta: \mathbb{R} \rightarrow[0,1]$ denote a smooth cut-off function with uniformly bounded derivative, $\left|\beta^{\prime}(r)\right| \leq 2$, such that $\beta(r)=0$ if $r \leq 1$ and $\beta(r)=1$ for $r \geq 2$. The pregluing of $(u, \mathbf{j})$ with parameter $\rho$ is the smooth map,

$$
u_{\rho} \equiv \operatorname{pgl}^{X}(u, \mathbf{j}, \rho): \Sigma_{\rho} \rightarrow X,
$$

defined as follows: for each $v \in V$, for every $v^{\prime} \in V$ such that $v E v^{\prime}$,

$$
u_{\rho}(z):= \begin{cases}p_{v v^{\prime}}:=u_{v}\left(y_{v, v^{\prime}}\right)=u_{v^{\prime}}\left(y_{v, v^{\prime}}\right), & \text { if } z \in D_{f}\left(r_{v v^{\prime}}^{1 / 4}\right) \backslash D_{f}\left(r_{v v^{\prime}}^{3 / 4}\right), \\ \exp _{p_{v v^{\prime}}}\left(\beta\left(|z| / r_{v v^{\prime}}^{1 / 4}\right)\left(\exp _{p_{v v^{\prime}}}^{-1}, u_{v}(z)\right)\right), & \text { if } z \in D_{f}\left(2 r_{v v^{\prime}}^{1 / 4}\right) \backslash D_{f}\left(r_{v v^{\prime}}^{1 / 4}\right), \\ \exp _{p_{v v^{\prime}}}\left(\beta\left(r_{v v^{\prime}}^{3 / 4} /|z|\right)\left(\exp _{p_{v v^{\prime}}}^{-1}, u_{v^{\prime}}(\rho / z)\right)\right), & \text { if } z \in D_{f}\left(r_{v v^{\prime}}^{3 / 4}\right) \backslash D_{f}\left(r_{v v^{\prime}}^{3 / 4} / 2\right)\end{cases}
$$

and $u_{\rho}(z)$ coincides with $u_{v}(z)$ away from the annuli above. Here, we need to assume $\rho$ small enough, so that the discs $D_{f}\left(4 r_{v v^{\prime}}^{1 / 4}\right)$ are sent under $u_{v}$ in a normal neighborhood of $p_{v v^{\prime}}$. Note that the mapping $\mathrm{pgl}^{X}$ is continuous with respect to $\rho$.

Estimates from the pregluing. The following estimates are all standard and their proofs can be found in [3] (Section 10) or in [17] (Section 10.3 and the proof of Lemma 10.6.3). The first estimate tells that $u_{\rho}$ is approximately $J$-holomorphic. The second gives a quadratic estimate ensuring existence 
and unicity of the gluing map. Finally, the third is needed to derive that the constructed gluing map is a local diffeomorphism.

Lemma 6.1. Let $p>2$ an integer, and $U_{X}$ a proper open subset of $\mathcal{M}_{\mathcal{S}_{X}}(X)$. There is a uniform (with respect to $\rho$ ) constant $c^{X}$ such that for every $(u, \mathbf{j}) \in$ $U_{X}$ :

$$
\left\|d u_{\rho}\right\|_{L^{\infty}} \leq c^{X}, \quad\left\|\bar{\partial}_{J} u_{\rho}\right\|_{L^{p}} \leq c^{X}|\rho|^{1 / 2 p} .
$$

Consequently, there is a uniform constant $c_{1}^{X}$ such that

$$
\left\|N_{u_{\rho}}^{X}\left(\xi_{1}\right)-N_{u_{\rho}}^{X}\left(\xi_{2}\right)\right\|_{L^{p}} \leq c_{1}^{X}\left(\left\|\xi_{1}\right\|_{W^{1, p}}+\left\|\xi_{2}\right\|_{W^{1, p}}\right)\left\|\xi_{1}-\xi_{2}\right\|_{W^{1, p}},
$$

where $N_{u}^{X}$ denotes the non-linear part in the Taylor expansion of $\bar{\partial}_{J}$ at $u$ :

$$
N_{u}^{X}(\xi)=\bar{\partial}_{J} \exp _{u} \xi-\bar{\partial}_{J} u-D_{u}^{X} \xi, \quad \xi \in W^{1, p}\left(u^{*} T X\right) .
$$

Finally, let $u_{t}:=\left\{\left(u_{v t}, u_{v^{\prime} t}\right)\right\}_{v E v^{\prime}}, t \in[0, v)$ be a path in $\mathcal{B}_{X}^{1, p}$, with

$$
\zeta \equiv\left\{\left(\zeta_{v}, \zeta_{v^{\prime}}\right)\right\}_{v E v^{\prime}}:=\left.\frac{d}{d t}\right|_{t=0} u_{t}
$$

Let $u_{\rho, t}$ denote the corresponding path of preglued curves and set $\zeta_{\rho}:=$ $\left.\frac{d}{d t}\right|_{t=0} u_{\rho, t}$. There is a uniform constant $\widetilde{c}^{X}$ such that

$$
\left\|\zeta_{\rho}\right\|_{W^{1, p}} \leq \widetilde{c}^{X}\|\zeta\|_{W^{1, p}}, \quad\left\|D_{u_{\rho, 0}}^{X} \zeta_{\rho}\right\|_{L^{p}} \leq\left\|D_{u_{0}}^{X} \zeta\right\|_{L^{p}}+\widetilde{c}^{X}|\rho|^{1 / 2 p}\|\zeta\|_{C^{1}} .
$$

In particular, if $u_{t}$ is a path of holomorphic curves, i.e., if $\zeta$ is in the kernel of $D_{u_{0}}^{X}$, the first term of the right handside of the second inequality vanishes.

Step 2: Right inverses. The gluing operation will give a holomorphic map with domain $\Sigma_{\rho}$ obtained by perturbing the preglued map in directions that are transverse to the kernel of $D_{u_{\rho}}^{X}$, i.e., lying in the image of a uniformly bounded family $Q_{u_{\rho}}^{X}$ of right inverses for $D_{u_{\rho}}^{X}$. Below we sketch the proof of the following:

Proposition 6.1 ([17], Proposition 10.5.1). Let $p>2$ and $U_{X}$ as before. There exists a uniformly bounded family $Q_{u_{\rho}}^{X}$ of right inverses for $D_{u_{\rho}}^{X}$, i.e., there is a uniform constant $c^{X}$ such that for every $(\mathbf{j}, u) \in U_{X}$ :

$$
\left\|Q_{u_{\rho}}^{X} \eta\right\|_{W^{1, p}} \leq c^{X}\|\eta\|_{L^{p}} .
$$

Proof. First, one constructs an interpolation $\mathbf{w}_{\rho}:=\left\{u_{v, \rho}: \Sigma_{v} \rightarrow X\right\}_{\{v \in V\}}$ between $u$ and $u_{\rho}$ as follows: for $v \in V$,

$$
u_{v, \rho}:= \begin{cases}u_{\rho}(z), & \text { if } z \in \Sigma_{v} \backslash \bigcup_{\left\{v^{\prime} \mid v E v^{\prime}\right\}} D_{f}\left(r_{v v^{\prime}}^{1 / 4}\right), \\ u_{v}\left(y_{v, v^{\prime}}\right)=p_{v v^{\prime}}, & \text { otherwise. }\end{cases}
$$

where $f$ is the half-edge in $\operatorname{pr}^{-1}(v)$ associated to the edge between $v$ and $v^{\prime}$. As as $\rho$ goes to 0 , the "flattened" map $u_{v, \rho}$ converges to $u_{v}$ in $W^{1, p}$ norm. Thus, $D_{u_{v, \rho}}^{X}$ converges to $D_{u_{v}}^{X}$ in the operator norm, and since $D_{u_{v}}^{X}$ is 
surjective then $D_{u_{v, \rho}}^{X}$ also is. As a result, $D_{\mathbf{w}_{\rho}}^{X}$ is surjective. Therefore, $D_{\mathbf{w}_{\rho}}^{X}$ has right inverse $Q_{\mathbf{w}_{\rho}}^{X}$, which can be chosen uniquely by requiring that its image lies in the $L^{2}$-orthogonal complement of $\operatorname{ker} D_{\mathbf{w}_{\rho}}^{X}$. Moreover, $Q_{\mathbf{w}_{\rho}}^{X}$ is uniformly bounded. Next, we construct a quasi-right inverse $R_{u_{\rho}}^{X}$ for $D_{u_{\rho}}^{X}$ out of $Q_{\mathbf{w}_{\rho}}^{X}$, defined by

$$
R_{u_{\rho}}^{X}: \mathcal{E}_{X, u_{\rho}}^{p} \rightarrow \mathcal{X}_{X, u_{\rho}}^{1, p}, \quad \eta \mapsto \Gamma \circ Q_{w_{\rho}}^{X} \circ \Lambda(\eta) .
$$

We give the definitions of $\Lambda$ and $\Gamma$. For $v E v^{\prime}$, with corresponding edge $e_{f, f^{\prime}}$, recall that $\Sigma_{\rho}\left(f, f^{\prime}\right)$ is obtained by patching together the annuli $\mathcal{A}_{f}$ and $\mathcal{A}_{f^{\prime}}$. Consider the circles in $\Sigma_{\rho}$ corresponding to the circles

$$
\left\{-R_{v v^{\prime}} / 2\right\} \times \mathbb{R} / 2 \pi \mathbb{Z} \cong\left\{R_{v v^{\prime}} / 2\right\} \times \mathbb{R} / 2 \pi \mathbb{Z},
$$

and let $\mathcal{C}$ be the union of all these circles. Consider the biholomorphism (onto its image),

$$
\pi_{\rho}: \Sigma_{\rho} \backslash \mathcal{C} \rightarrow \Sigma
$$

defined as the identity map outside the annuli $\mathcal{A}_{f, f^{\prime}}:=\mathcal{A}_{f} \sim \mathcal{A}_{f^{\prime}}$ and given by:

Observe that

$$
\pi_{\rho}\left(z_{f}, z_{f^{\prime}}\right):= \begin{cases}z_{f}, & \text { if }\left|z_{f}\right|>\left|z_{f^{\prime}}\right| \\ z_{f^{\prime}}, & \text { if }\left|z_{f^{\prime}}\right|>\left|z_{f}\right|\end{cases}
$$

$$
u_{\rho}:= \begin{cases}\mathbf{w}_{\rho} \circ \pi_{\rho}, & \text { on } \Sigma_{\rho} \backslash \mathcal{C} \\ u_{v}\left(y_{v v^{\prime}}\right)=u_{v^{\prime}}\left(y_{v^{\prime} v}\right)=p_{v v^{\prime}}, & \text { on } \mathcal{C} .\end{cases}
$$

Now set

$$
\begin{aligned}
& \Lambda: L^{p}\left(\Lambda_{J}^{0,1}\left(\Sigma_{\rho}, u_{\rho}^{*} T X\right)\right) \rightarrow L^{p}\left(\Lambda_{J}^{0,1}\left(\Sigma, w_{\rho}^{*} T X\right)\right), \\
& \eta \mapsto \begin{cases}\left(\pi_{\rho}^{*}\right)^{-1} \eta, & \text { on } \operatorname{Im}\left(\pi_{\rho}\right), \\
0, & \text { otherwise. }\end{cases}
\end{aligned}
$$

Next, we define $\Gamma$. Set $\xi_{v, v^{\prime}}:=\xi\left(y_{v, v^{\prime}}\right)$ and $\beta_{r_{v v^{\prime}}}(z):=\beta\left(4 \log |z| / \log r_{v v^{\prime}}\right)$, where $\beta$ is a cut-off function as before. Then $\Gamma$ is an interpolation between $\xi_{v}$ and $\xi_{v^{\prime}}$ :

$$
\Gamma: W^{1, p}\left(w_{\rho}^{*} T X\right) \rightarrow W^{1, p}\left(u_{\rho}^{*} T X\right), \quad \xi:=\left\{\xi_{v}\right\}_{v \in V} \mapsto \xi_{\rho},
$$

where

$\xi_{\rho}=\left\{\begin{array}{l}\xi_{v}\left(z_{f}\right)+\beta_{r_{v v^{\prime}}}\left(z_{f}\right)\left(\xi_{v^{\prime}}\left(z_{f^{\prime}}\right)-\xi_{v, v^{\prime}}\right), \quad \text { when } \quad\left|z_{f^{\prime}}\right| \leq\left|z_{f}\right| \leq\left|r_{v v^{\prime}}\right|^{1 / 4}, \\ \xi_{\rho}=\xi(z) \text { otherwise. }\end{array}\right.$

It follows from the estimates (6.8) in Lemma 6.2 below that the maps are $D_{u_{\rho}}^{X} R_{u_{\rho}}^{X}$ is invertible for small enough gluing parameters. Consequently,

$$
Q_{u_{\rho}}^{X}:=R_{u_{\rho}}^{X}\left(D_{u_{\rho}}^{X} R_{u_{\rho}}^{X}\right)^{-1}
$$


is the unique right inverse for $D_{u_{\rho}}^{X}$ having the same image as $R_{u_{\rho}}^{X}$.

Right inverses estimates. The first estimate below gives the invertibility of $D_{u_{\rho}}^{X} R_{u_{\rho}}^{X}$. The second estimates how the right inverses $Q_{u_{\rho}}^{X}$ vary along a path in $\mathcal{B}_{X}^{1, p}$ of preglued maps, which is needed to show that the gluing map is a local diffeomorphism. For detailed proofs we refer to Proposition 10.5.1 and Lemma 10.6.2 in [17].

Lemma 6.2. Let $p>2$. The operator $R_{u_{\rho}}^{X}$ depends smoothly on $(\mathbf{j}, u)$ and there are constants $C^{X}$ and $\widetilde{C}^{X}$ independent of $\rho$, such that:

$$
\left\|D_{u_{\rho}}^{X} R_{u_{\rho}}^{X} \eta-\eta\right\|_{L^{p}} \leq \frac{C^{X}}{|\log | \rho||^{1-1 / p}}\|\eta\|_{L^{p}}, \quad\left\|R_{u_{\rho}}^{X} \eta\right\|_{W^{1, p}} \leq \frac{\widetilde{C}^{X}}{2}\|\eta\|_{L^{p}}
$$

Moreover, let $\left\{u_{t}\right\}_{t \in[0, v)}$ be a path of stable maps, with $\zeta:=\left.\frac{d}{d t}\right|_{t=0} u_{t}$. Let $\left\{u_{\rho, t}\right\}_{t \in[0, v)}$ be the corresponding path of preglued maps, with $u_{\rho, 0}:=u_{\rho}$. there is a uniform constant $\bar{c}^{X}$ such that

$$
\left\|\left.\frac{d}{d t}\right|_{t=0} Q_{u_{\rho, t}}^{X}\right\| \leq \bar{c}^{X}\|\zeta\|_{W^{1, p}} .
$$

Proof. We only give the proof of estimate (6.9).

$$
\left.\frac{d}{d t}\right|_{t=0} Q_{u_{\rho, t}}^{X}=\left(\left.\frac{d}{d t}\right|_{t=0} R_{u_{\rho, t}}^{X}\right)\left(D_{u_{\rho}}^{X} R_{u_{\rho}}^{X}\right)^{-1}+\left.R_{u_{\rho}}^{X} \frac{d}{d t}\right|_{t=0}\left(D_{u_{\rho, t}}^{X} R_{u_{\rho, t}}^{X}\right)^{-1} .
$$

On the one hand,

$$
\left\|\left.\frac{d}{d t}\right|_{t=0} R_{u_{\rho, t}}^{X}\right\|=\left\|\Gamma\left(\left.\frac{d}{d t}\right|_{t=0} Q_{w_{t}}^{X}\right) \Lambda\right\| \leq M\|\zeta\|_{W^{1, p}}
$$

since $\left\|\left.\frac{d}{d t}\right|_{t=0} Q_{w_{\rho, t}}^{X}\right\|$ is bounded above by $C\|\zeta\|_{W^{1, p}}$ for some positive constant $C$ (see [17], Lemma 10.6.2). On the other hand,

$$
\begin{aligned}
& \left.\left(D_{u_{\rho}}^{X} R_{u_{\rho}}^{X}\right) \frac{d}{d t}\right|_{t=0}\left(D_{u_{\rho}, t}^{X} R_{u_{\rho, t}}^{X}\right)^{-1} \\
& \quad=-\left(D_{u_{\rho}}^{X} R_{u_{\rho}}^{X}\right)^{-1}\left(\left.\frac{d}{d t}\right|_{t=0} D_{u_{\rho}, t}^{X} R_{u_{\rho}}^{X}+\left.D_{u_{\rho}}^{X} \frac{d}{d t}\right|_{t=0} R_{u_{\rho, t}}^{X}\right) .
\end{aligned}
$$

But the norm of derivative of $D_{u_{\rho, t}}^{X}$ is bounded and the estimate follows.

Step 3: the gluing map $G l_{\mathcal{S}_{X}}$. Let $U_{X}$ be a proper open subset of $\mathcal{M}_{\mathcal{S}_{X}}(X)$. For $\delta_{X} \in \mathbb{R}^{+}$, let $B_{\delta_{X}}(0)$ denote the $\delta_{X}$-neighborhood around the 0-section in $\mathcal{E}_{X}^{p}(J)$. Also, let $B_{\delta_{X}, u_{\rho}}(0)$ denote the corresponding ball in $\mathcal{E}_{X, u_{\rho}}^{p}(J)$. Then, for each $(u, \mathbf{j}, \rho)$ we can find a unique element

$$
f^{X}(u, \mathbf{j}, \rho) \in B_{\delta_{X}, u_{\rho}}(0) \subset L^{p}\left(\Lambda_{J}^{0,1}\left(S^{2}, u_{\rho}^{*} T X\right)\right)
$$

verifying:

$$
\bar{\partial}_{J}\left(\exp _{u_{\rho}} Q_{u_{\rho}}^{X}\left(f^{X}(u, \mathbf{j}, \rho)\right)\right)=0 .
$$


The gluing map is then defined by

$$
\begin{aligned}
G l_{\mathcal{S}_{X}}: \mathcal{L}_{\mathcal{S}_{X}, \epsilon_{X}, U_{X}}^{*} & \rightarrow \mathcal{M}_{0, l}(X, A), \\
(u, \mathbf{j}, \rho) & \mapsto\left(\mathbf{j}_{\rho}, \exp _{u_{\rho}} Q_{u_{\rho}}^{X}\left(f^{X}(u, \mathbf{j}, \rho)\right)\right),
\end{aligned}
$$

for some positive constant $\epsilon_{X}$ given by the Implicit Function Theorem below. Note that we directly have the commutativity (6.2). Moreover, in reality, the gluing map is defined on local uniformizing system for $\mathcal{L}_{\mathcal{S}_{X}, \epsilon_{X}, U_{X}}$, but since $\mathcal{S}_{X}$ and $\mathcal{S}$ are stable, the gluing is invariant under $\operatorname{Aut}(\mathbf{j})$, hence welldefined after quotient by the reparameterizations. Existence and unicity of $f^{X}(u, \mathbf{j}, \rho)$ are given by the following standard parametric version of the implicit function theorem (Theorem 9.5 in [3], Theorem 3.5.2 in [17]).

Implicit Function Theorem. Let $p>2$ and let $c_{1}^{X}$ denote the positive constant in (6.4). There is a constant $\epsilon_{X}$ such that, for every $(u, \mathbf{j}, \rho) \in$ $\mathcal{L}_{\mathcal{S}_{X}, \epsilon_{X}, U_{X}}$ we have a uniform positive constants $\delta_{X}, \epsilon_{1}^{X}$ and $c_{2}^{X}$, verifying:

$$
\left\|\bar{\partial}_{J} u_{\rho}\right\|_{L^{p}} \leq \epsilon_{1}^{X}, \quad\left\|Q_{u_{\rho}}^{X}\right\| \leq c_{2}^{X}, \quad \epsilon_{1}^{X}<\delta_{X} / 4, \quad \epsilon_{1}^{X}<\left(8 c_{1}^{X}\left(c_{2}^{X}\right)^{2}\right)^{-1},
$$

and we have a map,

$$
f^{X}: \mathcal{L}_{\mathcal{S}_{X}, \epsilon_{X}, U_{X}} \rightarrow B_{\delta_{X}}(0),
$$

which is continuous in the $\rho$ coordinate and smooth in the $(u, \mathbf{j})$ coordinate, such that $f^{X}(u, \mathbf{j}, \rho)$ is the unique solution to (6.11). Furthermore,

$$
\left\|f^{X}(u, \mathbf{j}, \rho)\right\|_{L^{p}}<2 \epsilon_{1}^{X} .
$$

Proof. The constants $\epsilon_{1}^{X}$ and $c_{1}^{X}$ are given by the estimates in Lemma 6.1, while $c_{2}^{X}$ is given by Proposition 6.6. Consider the Fredholm fibered map:

$$
\begin{aligned}
& F^{X}:\left(\operatorname{pgl}^{X}\right)^{*} T \mathcal{B}_{X} \rightarrow \mathcal{E}_{X}^{p}(J), \\
& ((u, \mathbf{j}, \rho), \xi) \mapsto F_{(u, \mathbf{j}, \rho)}^{X}(\xi):=\Phi_{X, u_{\rho}}^{-1}(\xi) \bar{\partial}_{\mathbf{j}_{\rho}, J}\left(\exp _{u_{\rho}} \xi\right)
\end{aligned}
$$

where $\Phi_{X}$ is the parallel transport induced by the Hermitian connection induced from the $\mathrm{L}-\mathrm{C}$ connection $\nabla^{T X}$ with respect to $g_{J}$. When $\xi=0$, this map coincides with $\bar{\partial}_{\mathbf{j}_{\rho}, J} u_{\rho}$ so that

$$
D F_{(u, \mathbf{j}, \rho)}^{X}(0)(\xi)=D_{u \rho}^{X} \xi .
$$

For every $x:=(u, \mathbf{j}, \rho) \in \mathcal{L}_{\mathcal{S}_{X}, \epsilon_{X}, U_{X}}$, we want to find $f^{X}(x)$ in $\mathcal{E}_{X, x}^{p}(J)$ such that $F^{X}\left(Q_{x}^{X} f^{X}(x)\right)=0$, in other words such that

$$
0=F_{x}^{X}(0)+f^{X}(x)+N_{x}^{X}\left(Q_{x}^{X} f^{X}(x)\right),
$$

where $N_{x}^{X}$ is the non-linear term of the expansion of $F_{x}^{X}$ around $\xi \equiv 0$. Consider the family of operators

$$
H_{x}: \mathcal{E}_{X, x}^{p}(J) \rightarrow \mathcal{E}_{X, x}^{p}(J), \quad \eta \rightarrow-F_{x}^{X}(0)-N_{x}^{X}\left(Q_{x}^{X} \eta\right) .
$$


We have

$$
\left\|H_{x}(\eta)\right\|_{L^{p}} \leq\left\|F_{x}^{X}(0)\right\|_{L^{p}}+\left\|N_{x}^{X}\left(Q_{x}^{X} \eta\right)\right\|_{L^{p}} \leq \epsilon_{1}^{X}+c_{1}^{X}\left(c_{2}^{X}\right)^{2}\|\eta\|_{L^{p}}^{2},
$$

so that $H$ maps the ball $B_{\delta_{X}}(0)$ into itself whenever $\epsilon_{1}^{X}+c_{1}^{X}\left(c_{2}^{X}\right)^{2} \delta_{X}^{2} \leq \delta_{X}$. This is realized when $\epsilon_{1}^{X}<\delta_{X} / 4$ and $4 c_{1}^{X}\left(c_{2}^{X}\right)^{2} \epsilon_{1}^{X}<1 / 2$. Furthermore,

$$
\begin{aligned}
\left\|H_{x}\left(\eta_{1}\right)-H_{x}\left(\eta_{2}\right)\right\|_{L^{p}} & =\left\|N_{x}^{X}\left(Q_{x}^{X} \eta_{1}\right)-N_{x}^{X}\left(Q_{x}^{X} \eta_{2}\right)\right\|_{L^{p}} \\
& \leq 2 c_{1}^{X}\left(c_{2}^{X}\right)^{2} \delta_{X}\left\|\eta_{1}-\eta_{2}\right\|_{L^{p}} \\
& <\left\|\eta_{1}-\eta_{2}\right\|_{L^{p}}
\end{aligned}
$$

Thus, $H$ is a contraction map so that existence and uniqueness follow. One can further show that the map $H$ defines a contraction from $B_{2 \epsilon_{1}^{X}}(0)$ to itself when $4 c_{1}^{X}\left(c_{2}^{X}\right)^{2} \epsilon_{1}^{X}<1 / 2$. This gives the estimate $\left\|f^{X}(x)\right\|_{L^{p}}<2 \epsilon_{1}^{X}$. Smoothness of $f^{X}$ with respect to $(\mathbf{j}, u)$ follows from implicit function theorem since $D F_{x}^{X}$ is an isomorphism from $Q^{X}\left(\mathcal{E}_{X}^{p}(J)\right)$ to $\mathcal{E}_{X}^{p}(J)$.

Next, we prove that $G l_{\mathcal{S}_{X}}$ is a local diffeomorphism. We will in fact show that for every fixed $(\mathbf{j}, \rho)$, the gluing map $G l_{\mathcal{S}_{X}}(., \mathbf{j}, \rho)$ is a local diffeomorphism. Then the statement follows since, as the gluing map $g l_{\mathcal{S}}$ is a local diffeomorphism by our stability assumption, we can treat the pairs $(\mathbf{j}, \rho)$ as actual parameters (cf [3] Section 12, [20] Section 3). Without loss of generality we can assume that $U_{X}$ is the preimage under $\mathcal{F}_{X}$ of a neighborhood of $\mathbf{j}_{0} \in \mathcal{M}_{\mathcal{S}}$. Fix $(\mathbf{j}, \rho)$, and let $u_{\rho 0}$ be the pregluing with parameter $\rho$ of some $u_{0}$ in the preimage of $\mathbf{j}$. Let $W$ be an open neighborhood of 0 in $W^{1, p}\left(S^{2}, u_{\rho}^{*} T X\right)$. Also, let $U_{1}$ be the image of $U_{X} \cap \mathcal{F}_{X}^{-1}(\mathbf{j})$ under $\operatorname{pgl}^{X}(., \mathbf{j}, \rho)$. Decompose $G l_{\mathcal{S}_{X}}(., \mathbf{j}, \rho)$ as follows:

$$
U_{1} \stackrel{1 \times f_{\mathbf{j}, \rho}^{X}}{\longrightarrow} U_{1} \times B_{\delta_{X}}(0) \stackrel{\Phi}{\longrightarrow} W \stackrel{\exp _{u_{\rho_{0}}}}{\longrightarrow} \mathcal{M}_{0, l}(P),
$$

where $\Phi\left(u_{\rho}, \eta\right):=\xi_{\rho}+Q_{u_{\rho}}^{X} \eta$, where $\exp _{u_{\rho_{0}}} \xi_{\rho}=u_{\rho}$, and where $f_{\mathbf{j}, \rho}^{X}(u):=$ $f^{X}(u, \mathbf{j}, \rho)$. Also, for any path $u_{\rho_{t}}$ starting at $u_{\rho_{0}}$, with derivative $\xi$ at $t=0$, differentiating the equation

$$
0=\bar{\partial}_{J} u_{\rho_{t}}+f^{X}\left(u_{\rho_{t}}\right)+N_{u_{\rho_{t}}}^{X}\left(Q_{u_{\rho_{t}}}^{X} f^{X}\left(u_{\rho_{t}}\right)\right)
$$

and using the estimates proved so far, one obtains the estimate:

$$
\left\|\frac{d f^{X}}{d \xi}\right\|_{L^{p}}<C r^{1 / 2 p}\|\xi\|_{W^{1, p}} .
$$

This ensures that for small enough gluing parameter, the differential of the gluing map is well defined. Therefore, it suffices to check that $\Phi$ is a diffeomorphism. To prove this, we identify $W^{1, p}\left(S^{2}, u_{\rho_{0}}^{*} T X\right)$ with $\operatorname{ker} D_{u_{\rho_{0}}}^{X} \oplus L^{p}\left(S^{2}, u_{\rho_{0}}^{*} T X\right)$ via the map

$$
\xi \mapsto\left(\left(I d-Q_{u_{\rho_{0}}}^{X} D_{u_{\rho_{0}}}^{X}\right) \xi, D_{u_{\rho_{0}}}^{X} \xi\right),
$$


and then rewrite $\Phi$ as

$$
\Phi\left(u_{\rho}, \eta\right)=\left(\left(I d-Q_{u_{\rho_{0}}}^{X} D_{u_{\rho_{0}}}^{X}\right)\left(\xi_{u_{\rho}}+Q_{u_{\rho}}^{X} \eta\right), D_{u_{\rho_{0}}}^{X}\left(\xi_{\rho}+Q_{u_{\rho}}^{X} \eta\right)\right) .
$$

Then, $D^{X} \Phi_{\left(u_{\rho}, \eta\right)}=I d+K_{\left(u_{\rho}, \eta\right)}$ where

$K_{\left(u_{\rho}, \eta\right)}(\xi, \zeta)=\left(\begin{array}{cc}\left(I d-Q_{u_{\rho_{0}}}^{X} D_{u_{\rho_{0}}}^{X}\right)\left(\xi+\frac{d Q_{u_{\rho}}^{X}}{d \xi} \eta\right)-\xi & \left(I d-Q_{u_{\rho_{0}}}^{X} D_{u_{\rho_{0}}}^{X}\right) Q_{u_{\rho}}^{X} \zeta \\ D_{u_{\rho_{0}}}^{X}\left(\xi+\frac{d Q_{u_{\rho}}^{X}}{d \xi} \eta\right) & \left(Q_{u_{\rho_{0}}}^{X} D_{u_{\rho_{0}}}^{X}-I d\right) \zeta\end{array}\right)$.

Hence, we deduce from Corollary 6.1 and Lemma 6.9, that for proper subset $U_{1}^{\prime} \subset U_{1}$, and for $\delta_{1}<\delta_{X}$, the operator $D^{X} \Phi_{\left(u_{\rho}, \eta\right)}$ is invertible for any $\left(u_{\rho}, \eta\right) \in U^{\prime} \times B_{\delta_{1}}(0)$, and that

$$
\left\|D^{X} \Phi_{\left(u_{\rho}, \eta\right)}\right\| \leq 2 .
$$

For the injectivity of $\Phi$, let $N\left(\xi_{\rho}, \eta\right)$ be the non-linear part in the expansion of $\Phi\left(u_{\rho}, \eta\right)$ around $\left(u_{\rho_{0}}, 0\right)$. Then,

$$
N^{X}\left(\xi_{\rho}, \eta\right)=\left(\left(I d-Q_{u_{\rho_{0}}}^{X} D_{u_{\rho_{0}}}^{X}\right) Q_{u_{\rho}}^{X} \eta, D_{u_{\rho_{0}}}^{X} Q_{u_{\rho}}^{X} \eta-\eta\right) .
$$

Assume that $\Phi\left(u_{\rho_{1}}, \eta_{1}\right)=\Phi\left(u_{\rho_{2}}, \eta_{2}\right)$, for $\left(u_{\rho_{1}}, \eta_{1}\right) \neq\left(u_{\rho_{2}}, \eta_{2}\right)$. Then

$$
\left\|D^{X} \Phi_{\left(u_{\rho_{0}}, 0\right)}\left(\xi_{\rho_{1}}-\xi_{\rho_{2}}, \eta_{1}-\eta_{2}\right)\right\|_{L^{p}}=\left\|N^{X}\left(\xi_{\rho_{1}}, \eta_{2}\right)-N^{X}\left(\xi_{\rho_{2}}, \eta_{2}\right)\right\|_{L^{p}} .
$$

But, by standard arguments the right handside is bounded above by

$$
C\left(\left\|\left(\xi_{\rho_{1}}, \eta_{1}\right)\right\|_{W^{1, p}}+\left\|\left(\xi_{\rho_{2}}, \eta_{2}\right)\right\|_{W^{1, p}}\right)\left\|\left(\xi_{\rho_{1}}-\xi_{\rho_{2}}, \eta_{1}-\eta_{2}\right)\right\|_{W^{1, p}}
$$

for some positive constant $C$. Since $\left\|\left(\xi_{\rho_{1}}-\xi_{\rho_{2}}, \eta_{1}-\eta_{2}\right)\right\|_{W^{1, p}}>0$ by assumption, we have

$$
0<\left\|D^{X} \Phi_{\left(u_{\rho_{0}}, 0\right)}\right\|_{L^{p}} \leq C\left(\left\|\left(\xi_{\rho_{1}}, \eta_{1}\right)\right\|_{W^{1, p}}+\left\|\left(\xi_{\rho_{2}}, \eta_{2}\right)\right\|_{W^{1, p}}\right),
$$

which is impossible for small enough $\left(\xi_{\rho_{i}}, \eta_{i}\right), i=1,2$.

As such, the construction above still applies when the domain is not stable, but the obtained gluing maps are only defined at the level of parameterized maps, i.e., before quotienting by $\operatorname{Aut}(\mathbf{j})$, even though these maps are $\operatorname{Aut}(u, \mathbf{j})$ equivariant. The reason is that so far we have parameterized our gluing maps according to the gluing for the domains, but the gluing for nodal surfaces is neither injective, nor a local diffeomorphism in the unstable case. Therefore, we cannot treat the gluing parameters $\rho$ as parameters. The problem is to find a slice for the action of the group of reparameterizations of the domain. 
6.1.3. Gluing unstable components. Let $(u, \mathbf{j}) \in \mathcal{M}_{\mathcal{S}_{X}}(X)$, and let $v \in$ $V$ be a $\pi_{p t_{*}}$-unstable component. Note that $|v|>2$ is possible. Following Chen-Li [3], we describe the notion of balanced component when $|v| \leq 2$. For the commodity of the readers, we furnish the details below, as it will be generalized to the case of general base $B$. Let $\mathbf{t r} \cong \mathbb{C} \subset G$ denote the subgroup of translations, and let $\mathbf{m} \cong \mathbb{C}^{*} \subset G$ denote the subgroup acting on $S^{2}=\mathbb{C} \cup\{\infty\}$ by complex multiplication. Then, the semi direct product $\mathcal{G}:=\mathbf{t} \ltimes \mathbf{m}$ acts on $\mathbb{C}$ by

$$
(m, t) \in \mathcal{G}, \quad(t, m) \cdot z:=m(z-t) .
$$

(1) Balanced maps. First assume $|v|=2$. Up to the action of $\mathbf{m}$, the component $v$ can be parameterized by $\mathbb{C P}^{1}$ with special points $0=[1: 0]$ and $\infty=[0: 1]$. Identify $\Sigma_{v} \backslash\{\infty\}$ with $\mathbb{C}$. The parameterization is balanced if:

$$
\frac{1}{2} \int_{|z| \leq 1}\|d u\|^{2} d \operatorname{vol} S^{2}=\hbar
$$

where $\hbar$ denotes the minimal energy of a non-constant pseudo-holomorphic map in $X$. Next, when $|v|=1$, up to the action of $\mathcal{G}$, the component $v$ can be parameterized by $\mathbb{C P}^{1}$ such that the special point is $\infty$. Identify $\Sigma_{v} \backslash\{\infty\}$ with $\mathbb{C}$. The parameterization is balanced if (6.13) holds and if the center of energy of $u$ is 0 :

$$
\int z\|d u\|^{2} d \operatorname{vol} S^{2}=0, \quad \text { where } z \in \mathbb{C} .
$$

We will say that $u$ is centered if it is the case. The $\pi_{p t_{*}}$-unstable component $\Sigma_{v}$ with balanced parameterization is called a balanced component. Recall that the reparameterizations of a $\pi_{p t_{*}}$-stable component is of finite order. Here, the reparameterizations of a balanced component is given by $S^{1}$. Note that the neighborhoods of the special points in a balanced parameterization for $\Sigma_{v}$ can be put in standard cylindrical coordinates: for $\infty$

$$
[0, \infty) \times \mathbb{R} / 2 \pi \mathbb{Z} \cong D^{*}\left(r_{\infty}\right):(s, t) \mapsto e^{s+i t},
$$

while for 0

$$
(-\infty, 0] \times \mathbb{R} / 2 \pi \mathbb{Z} \cong D^{*}\left(r_{0}\right):(s, t) \mapsto e^{s+i t} .
$$

To each unbalanced map $u_{v}: \Sigma_{v} \rightarrow X$ smoothly corresponds a unique element $\phi_{X}^{b}\left(u_{v}\right) \in \mathcal{G}$ consisting of the pair of translation and real dilation such that: the center of energy of

$$
u_{v}^{b}:=u_{v} \circ\left(\phi_{X}^{b}\left(u_{v}\right)\right)
$$

is zero; half the total energy of $u_{v}^{b}$ lies in the unit disc around zero. Let $\widetilde{\mathcal{M}}_{0, i}^{b}\left(X, A_{v}\right), i=1,2$, denote the sets of balanced $J$-holomorphic maps representing $A_{v}$ with one and two marked points. The map $u_{v} \mapsto u_{v}^{b}$ sends an 
orbit of $\mathcal{G}$ to an $S^{1}$ orbit where $S^{1}$ acts by rotations around the origin, hence we have the following natural identifications:

$$
\mathcal{M}_{0, i}(X, A) \cong \widetilde{\mathcal{M}}_{0, i}^{b}(X, A) / S^{1}, \quad i=1,2 .
$$

More generally, we say that $(u, \mathbf{j}) \in \widetilde{\mathcal{M}}_{\mathcal{S}_{X}}(X)$ is balanced if each of $\pi_{p t_{*}}$ unstable component $v$ with $|v| \leq 2$ is balanced. Let $\widetilde{\mathcal{M}}_{\mathcal{S}_{X}}^{b}(X)$ denote the subset of all balanced stable maps, and call it moduli space of balanced $J$-holomorphic maps for $\mathcal{S}_{X}$. On $\widetilde{\mathcal{M}}_{\mathcal{S}_{X}}^{b}(X)$ the action of the reparameterizations reduces to the action of $\mathrm{Aut}_{\text {red }}^{X}$ :

$$
\operatorname{Aut}_{\text {red }}^{X} \cong\left(S^{1}\right)^{\left|V_{b}\right|} \times \operatorname{Aut}\left(\mathcal{S}_{X}\right) .
$$

where $V_{b} \subset V$ denotes the subset of $\pi_{p t_{*}}$-unstable components $v$ with $|v| \leq 2$. From the discussion above we have the identification:

$$
\mathcal{M}_{\mathcal{S}_{X}}(X)=\widetilde{\mathcal{M}}_{\mathcal{S}_{X}}^{b}(X) / \mathrm{Aut}_{\text {red }}^{X} .
$$

Next we define the gluing maps for balanced curves.

(2) Gluing balanced maps. Let $\mathcal{S}:=S_{\pi_{p t_{*}}}\left(\mathcal{S}_{X}\right)$. Set $\mathcal{S}^{u}:=\mathcal{F}_{X}\left(\mathcal{S}_{X}\right)$, and let $\widetilde{\mathcal{M}}_{\mathcal{S}^{u}}$ denote the set of parameterized nodal curves having $\mathcal{S}^{u}$ as stratum data. Let $\widetilde{\mathcal{L}}_{\mathcal{S}^{u}}$ be the corresponding fiber bundle of gluing parameters. Also, let $\widetilde{\mathcal{L}}_{\mathcal{S}_{X}}$ denote the bundle $\mathcal{F}_{X}^{*} \widetilde{\mathcal{L}}_{\mathcal{S}^{u}}$ over $\widetilde{\mathcal{M}}_{\mathcal{S}_{X}}(X)$, and let $\widetilde{\mathcal{L}}_{\mathcal{S}_{X}}^{b}$ denote its restriction to $\widetilde{\mathcal{M}}_{\mathcal{S}_{X}}^{b}(X)$. The forgetful map $\bar{\pi}_{p t}^{\mathcal{S}_{P}}$ induces a map:

$$
\overline{\bar{\pi}}_{p t} \mathcal{S}_{X}: \widetilde{\mathcal{L}}_{\mathcal{S}_{X}} \rightarrow \widetilde{\mathcal{L}}_{\mathcal{S}}, \quad(u, \mathbf{j}, \rho) \mapsto\left(\bar{\pi}_{p t}^{\mathcal{S}_{X}}(u, \mathbf{j}), \rho^{\mathrm{st}}\right),
$$

where $\rho$ st leaves unchanged the gluing parameters between $\pi_{p t_{*}}$-stable components, forgets about all the gluing parameters of components lying in contracted branch and sends the gluing parameters of a connecting branch to the product of the parameters of the corresponding connecting chain.

Note that the group Aut ${ }_{\text {red }}^{X}$ acts naturally by rotations on $\widetilde{\mathcal{L}}_{\mathcal{S}_{X}}^{b}$. Since we consider balanced parameterizations, $\rho^{\text {st }}$ is invariant under the reparameterizations of $\mathbf{j}$, and $\overline{\bar{\pi}}_{p t} \mathcal{S}_{X}$ is well defined after quotient:

$$
\overline{\bar{\pi}}_{p t} \mathcal{S}_{X}: \mathcal{L}_{\mathcal{S}_{X}}^{b}:=\widetilde{\mathcal{L}}_{\mathcal{S}_{X}} / \mathrm{Aut}_{\text {red }}^{X} \rightarrow \mathcal{L}_{\mathcal{S}}
$$

Now, by hypothesis (SR), for any element of $\widetilde{\mathcal{M}}_{\mathcal{S}_{X}}^{b}(X)$, the linearization of the Cauchy-Riemann operator is surjective. It follows from Theorem 6.3 that for any proper open subset $U_{X}$ of $\widetilde{\mathcal{M}}_{\mathcal{S}_{X}}^{b}(X)$, that we may choose to be Aut ${ }_{\text {red }}^{X}$-invariant, there exists $\epsilon_{X}>0$ and a map

$$
\widetilde{G l}_{\mathcal{S}_{X}}^{b}: \widetilde{\mathcal{L}}_{\mathcal{S}_{X}, \epsilon_{X}, U_{X}}^{b *} \rightarrow \widetilde{\mathcal{M}}_{0, l}(X, A) .
$$




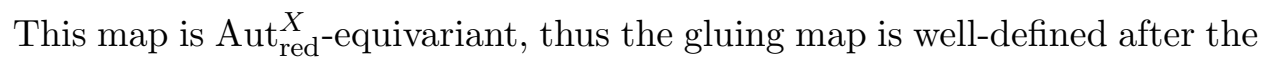
quotient by the action:

$$
G l_{\mathcal{S}_{X}}^{b}: \widetilde{\mathcal{L}}_{\mathcal{S}_{X}, \epsilon_{X}, U_{X}}^{b *} / \mathrm{Aut}_{\text {red }}^{X} \rightarrow \widetilde{\mathcal{M}}_{0, l}(X, A) .
$$

Note that the domain of $G l_{\mathcal{S}_{X}}^{b}(u, \mathbf{j}, \rho)$ is $g l_{\mathcal{S}^{u}}(\mathbf{j}, \rho)$. Since $\mathcal{S}^{u}$ is not stable, $g l_{\mathcal{S}^{u}}$ is not injective nor a local diffeomorphism and $\rho$ cannot be treated as a parameter anymore. Moreover, note that for $l \geq 3$, this gluing map takes value in $\mathcal{M}_{0, l}(X, A)$, while for $l<3$, one needs to make sure that the image of the gluing gives a slice for the action of the automorphisms of $S^{2}$ with less than three marked points.

Theorem 6.4 (Chen-Li [3], Theorems 12.2 and 12.10). $G l_{\mathcal{S}_{X}}^{b}$ is a local $C^{1}$-diffeomorphism. Furthermore,

$$
\bar{\pi}_{p t}^{\mathcal{S}_{X}} \circ G l_{\mathcal{S}_{X}}^{b}=g l_{\mathcal{S}} \circ \overline{\bar{\pi}}_{p t} \mathcal{S}_{X} .
$$

Proof. The gluing among $\pi_{*}$-unstable components is divided into two cases: (1) the gluing between a balanced component and a stable component; (2) the gluing between two balanced components. Let $\Sigma_{v}$ and $\Sigma_{v^{\prime}}$ be the two components to be glued at the edge $e_{f, f^{\prime}}$. We may write

$$
\Sigma_{v}=\left(S^{2}, z_{f} \equiv 0,\left\{x_{k}\right\}_{k=1, \ldots, m}\right) \quad \text { and } \quad \Sigma_{v^{\prime}}=\left(S^{2}, z_{f^{\prime}} \equiv \infty,\left\{x_{k}^{\prime}\right\}_{k=1, \ldots, m^{\prime}}\right) .
$$

Then (1) and (2) can be deduced from: (a) $\Sigma_{v}$ is $\pi_{*}$-stable while $\Sigma_{v^{\prime}}$ is not, $m \geq 3$ and $m^{\prime}=0$; (b) both components are $\pi_{*}$-unstable and $m=m^{\prime}=0$.

Case (a). For simplicity we forget about the marked points on $\Sigma_{v}$. Let $V_{z_{f}}$ denote a neighborhood of $z_{f}$, then $g l_{\mathcal{S}^{u}}$ sends the neighborhood $V_{z_{f}} \times \mathbb{C}^{*}$ of $\mathbf{j}$ to $\left(S^{2}, \infty\right)$. Let $\widetilde{N}_{u_{0}} \times V_{\rho_{0}}$ denote a neighborhood of $\left(u_{0}, \rho_{0}\right)$ in $\widetilde{\mathcal{M}}_{\mathcal{S}_{X}}(X) \times \mathbb{C}_{\mathbf{j}}^{*}$, in local coordinates for $\widetilde{\mathcal{L}}_{\mathcal{S}_{X}}$. We want to define a gluing map:

$$
G l: \frac{\widetilde{N}_{u_{0}} \times V_{\rho_{0}}}{\mathcal{G}} \rightarrow \mathcal{M}_{0, l}(X, A) .
$$

By choosing a proper slice for the action of $\mathcal{G}$ we can construct a well-defined gluing map, namely $G l_{\mathcal{S}_{X}}^{b}$, locally given by:

$$
G l_{\mathcal{S}_{X}}^{b}: N_{u_{0}, \mathbf{j}} \times V_{z_{f}} \times V_{\rho_{0}} \rightarrow \mathcal{M}_{0, l}(X, A),
$$

where $N_{u_{0}, \mathbf{j}}$ stands for an $S^{1}$ slice in $\widetilde{\mathcal{M}}_{\mathcal{S}_{X}}^{b}(X) \cap \mathcal{F}_{X}^{-1}(\mathbf{j})$ around $u_{0}$. To see that this map is a local diffeomorphism, we compare it to a gluing map already encountered. To do this, use the identification of $\mathbf{m}$ with $V_{\rho_{0}}$ to obtain a new map:

$$
G l_{1}: \frac{\tilde{N}_{u_{0}}}{\mathbf{t}} \times\left\{\rho_{0}\right\} \rightarrow \mathcal{M}_{0, l}(X, A) .
$$

Next, add two marked points on the second component, $\{0\}$ and $\{1\}$, in order to stabilize, and let $\mathcal{S}_{X}(2)$ denote the corresponding stratum data. 
Then $\widetilde{N}_{u_{0}}$ can be written as a product of $V_{z_{f}}$ with a neighborhood $N_{u_{0}, z_{f}}$ of $u_{0}$ in $\mathcal{F}_{X}^{-1}(\mathbf{j}) \cap \widetilde{\mathcal{M}}_{\mathcal{S}_{X}(2)}(X)$. Then, we use the natural identification between $\mathbf{t}$ and $V_{z_{f}}$ in order to obtain a gluing map $G l_{1}^{\prime}$ defined on $N_{u_{0}, z_{f}} \times\left\{z_{f}\right\} \times\left\{\rho_{0}\right\}$, which we know is a diffeomorphism. This map is in fact $C^{1}$-close to $G l_{\mathcal{S}_{X}}^{b}$ so that $G l_{\mathcal{S}_{X}}^{b}$ is a local diffeomorphism.

Case (b). We may assume that $z_{f}=0$ and $z_{f^{\prime}}=\infty$ in the balanced parameterizations. This time $\Sigma_{v}=\left(S^{2}, 0\right)$ and $\Sigma_{v^{\prime}}=\left(S^{2}, \infty\right)$. Furthermore,

$$
\operatorname{Aut}(\Sigma)=\mathcal{G}_{1} \times \mathcal{G}_{2}=\left(\mathbf{t}_{1} \ltimes \mathbf{m}_{1}\right) \times\left(\mathbf{t}_{2} \ltimes \mathbf{m}_{2}\right) .
$$

Again, $\operatorname{Aut}(\Sigma)$ acts on $\mathbb{C}_{\epsilon_{X}}^{*}$, and we set $\operatorname{Aut}_{v v^{\prime}}(\Sigma)$ to be the normal subgroup that fixes the gluing parameters under this action. Hence, $\operatorname{Aut}_{v v^{\prime}}(\Sigma)$ is isomorphic to $\mathbf{t}_{1} \times \mathbf{t}_{2} \times \mathbb{C}_{1}^{*}$, where $\mathbb{C}_{1}^{*}:=\Delta^{-1}(1)$ and

$$
\Delta: \mathbf{m}_{1} \times \mathbf{m}_{2} \rightarrow \mathbf{m}, \quad\left(m_{1}, m_{2}\right) \mapsto m_{1} m_{2} .
$$

The complementary of $\mathbb{C}_{1}^{*}$ in $\mathbf{m}_{1} \times \mathbf{m}_{2}$ is denoted by $\mathbb{C}_{2}^{*}$ and is naturally identified with $\mathbb{C}_{\epsilon_{X}}^{*}$. The map $G l_{\mathcal{S}_{X}}^{b}$ comes from a map on $\widetilde{\mathcal{M}}_{\mathcal{S}_{X}}(X)$ locally given by:

$$
\widetilde{G l}_{\mathcal{S}_{X}}: U_{u_{0}} \times V_{\rho_{0}} \rightarrow \widetilde{\mathcal{M}}_{0,0}(X, A),
$$

where $U_{u_{0}}$ denotes a $\mathcal{G}_{1} \times \mathcal{G}_{2}$-invariant neighborhood of $u_{0} \in \widetilde{\mathcal{M}}_{\mathcal{S}_{X}}(X)$, and $V_{\rho_{0}}$ is a neighborhood of $\rho_{0} \in \mathbb{C}_{\epsilon_{X}}^{*}$. The map $G l_{\mathcal{S}_{X}}^{b}$ is obtained by choosing an appropriate slice for the action of $\operatorname{Aut}(\Sigma)$ once we restrict ourselves to balanced maps. Quotienting by the automorphism group we get a map

$$
G l_{\mathcal{S}_{X}}: \frac{U_{u_{0}} \times V_{\rho_{0}}}{\mathcal{G}_{1} \times \mathcal{G}_{2}} \rightarrow \widetilde{\mathcal{M}}_{0,0}(X, A),
$$

which we would like to take values in $\widetilde{\mathcal{M}}_{0,0}(X, A) / \operatorname{Aut}\left(S^{2}\right)$. Using the identification between $V_{\rho_{0}}$ and a neighborhood of the identity in $\mathbb{C}_{2}^{*}$ we get a new map:

$$
G l_{\mathcal{S}_{X}, 1}: \frac{U_{u_{0}}}{\operatorname{Aut}_{v v^{\prime}}(\Sigma)} \times\left\{\rho_{0}\right\} \rightarrow \frac{\widetilde{\mathcal{M}}_{0,0}(X, A)}{\operatorname{Aut}\left(S^{2}\right)},
$$

which is a well-defined local diffeomorphism close to $G l_{\mathcal{S}_{X}}^{b}$. That this map is indeed well-defined follows since $\operatorname{Aut}_{v v^{\prime}}(\Sigma)$ and $\operatorname{Aut}\left(S^{2}\right)$ are locally diffeomorphic around the identity.

Proof of (6.18). First consider a contracted branch. It is connected to a unique $\pi_{*}$-stable component. The subgraph is a tree with a distinguished root that is attached to the $\pi_{*}$-stable component. We can parameterize each component $\Sigma_{v}$ of the branch by $\mathbb{C P}^{1}$, such that the special point closest to the root is given by $\infty=[0: 1]$. By gluing from the farthest component to the closest, it suffices to consider the case of only one component attached to a root. But in that case, the glued surface $\mathbf{j}_{\rho}$ is isomorphic to the domain of the root component for every small enough $\rho$, so that the forgetful map takes the corresponding glued maps to the same point. 
Now consider a connecting branch. Observe that we can treat the components that are not in the connecting chain in the same way as the the components of a contracted branch. Therefore we only consider the case where the connecting branch coincides with the connecting chain. This chain is connected to exactly two $\pi_{*}$-stable components. The subgraph is a tree with two distinguished components, a root and a top that are attached to $\pi_{*}$-stable components. We can parameterize each component $\Sigma_{v}$ of the branch by $\mathbb{C P}^{1}$, such that the special point that is the closest to the root is given by $\infty=[0: 1]$, and the point that is farthest is given by $0=[1: 0]$. Let $k$ denote the number of components of the connecting chain. By adding one marked point, say $\{1\}$, on every component of the chain, the resulting nodal Riemmann surface $\mathbf{j}^{\prime}$ becomes stable. Then for every $\rho$, the gluing $\Sigma_{\rho}$ is obtained from $\Sigma_{\rho}^{\prime}$ by forgetting the added marked points. Note that each such choice of point $\{1\}$ fixes one gluing parameter on each component of the chain. For $\rho=\left(\rho_{1}, \ldots, \rho_{k+1}\right)$, starting from the top, we can fix all gluing parameter to a fixed small $\rho_{0}$, except for the gluing parameter associated to the root and the $\pi_{*}$-stable component, which is given by $\widetilde{\rho}$ the product of all the $\rho_{i}$ 's. Now, $\Sigma_{\rho}^{\prime}$ and $\Sigma_{\widetilde{\rho}}^{\text {st }}$ are isomorphic since they are both realized by gluing on a cylinder of length $\sum \log \left|\rho_{i}\right|$.

6.2. Gluing for general $\boldsymbol{B}$. It is completely parallel to the special case $B=p t$ treated above. We mainly point out the differences. Let $\mathcal{S}_{P}:=$ $\left(V_{P}, \mathrm{Fl}_{P} ; \operatorname{pr}_{P}, \varrho_{P}\right)$ be a stable stratum data for $\overline{\mathcal{M}}_{0, l}(P, \sigma)$, and let $\mathcal{S}_{B}:=$ $\left(V_{B}, \mathrm{Fl}_{B} ; \mathrm{pr}_{B}, \varrho_{B}\right)$ be its image under $S_{\pi_{*}}$. Also let $\mathcal{S}$ denote the image of $\mathcal{S}_{P}\left(\right.$ or $\left.\mathcal{S}_{B}\right)$ under the forgetful map.

6.2.1. Pregluing. Let $(u, \mathbf{j})$ be a $J_{P}$-holomorphic stable map in $P$, representing the stratum data $\mathcal{S}_{P}$. We show that the pregluing of $(u, \mathbf{j})$ projects under $\pi$ to the pregluing of $(\pi(u), \mathbf{j})$ with same gluing parameter:

Lemma 6.3. For every $(u, \mathbf{j})$ stable map, and gluing parameter $\rho$ :

$$
\pi\left(u_{\rho}\right) \equiv \pi\left(p g l^{P}(u, \mathbf{j})\right)=p g l^{B}(\pi(u), \mathbf{j}) \equiv \pi(u)_{\rho} .
$$

Proof. Assume for simplicity in the notations that $|V|=2$ with elements $v$ and $v^{\prime}$, and let $e_{f, f^{\prime}}$ be the corresponding edge. Set

$$
\xi_{v}(z):=\exp _{p_{v v^{\prime}}}^{-1} u_{v}(z), \quad \xi_{v^{\prime}}(z):=\exp _{p_{v v^{\prime}}}^{-1} u_{v^{\prime}}\left(\rho_{v v^{\prime}} / z\right),
$$

and let $\beta^{+}$and $\beta^{-}$respectively denote the functions $\beta\left(|z| / r_{v v^{\prime}}^{1 / 4}\right)$ and $\beta\left(r_{v v^{\prime}}^{3 / 4} /|z|\right)$. From (2.1) we deduce that on $\Sigma_{v}$,

$$
\pi\left(u_{\rho}\right)= \begin{cases}\pi\left(u_{v}\right), & \text { if } z \in \Sigma_{v} \backslash D_{f}\left(2 r_{v v^{\prime}}^{1 / 4}\right), \\ \pi\left(p_{v v^{\prime}}\right)=\pi\left(u_{v}\left(y_{v, v^{\prime}}\right)\right)=\pi\left(u_{v^{\prime}}\left(y_{v, v^{\prime}}\right)\right), & \text { if } z \in D_{f}\left(r_{v v^{\prime}}^{1 / 4}\right) \backslash D_{f}\left(r_{v v^{\prime}}^{3 / 4}\right), \\ \exp _{\pi\left(p_{v v^{\prime}}\right)}\left(\beta^{+} \pi_{* p_{v v^{\prime}}} \xi_{v}(z)\right. & \text { otherwise. }\end{cases}
$$


We can rewrite this last expression as follows:

$$
\exp _{\pi\left(p_{v v^{\prime}}\right)}\left(\beta^{+} \exp _{\pi\left(p_{v v^{\prime}}\right)}^{-1}\left(\pi\left(u_{v}(z)\right)\right)+\beta^{-} \exp _{\pi\left(p_{v v^{\prime}}\right)}^{-1}\left(\pi\left(u_{v^{\prime}}\left(\rho_{v v^{\prime}} / z\right)\right)\right)\right),
$$

so that $\pi\left(u_{\rho}\right)$ coincides with the pregluing $\pi(u)_{\rho}$.

Remark 6.2. The map obtained is not the pregluing of the stabilized map $\bar{\pi}(u, \mathbf{j})$. If $u$ is only made of $\pi_{*}$-stable components, these two pregluings coincide. Furthermore, $u_{\rho}$ may not necessarily lie in the restriction $\left.P\right|_{\pi(u)}$, e.g if $u$ has only one $\pi_{*}$-stable component $u_{0}$. Nevertheless, we will see that the glued map projects to $\pi\left(u_{0}\right)$.

Let $p>2$ and let $(\mathbf{j}, u) \in \widetilde{\mathcal{M}}_{\mathcal{S}_{P}}(P)$. Here are some estimates that follow directly from Lemma 6.1 in the $B=p t$ case. From (6.3) there are uniform positive constants $c^{B}$ and $c^{v}$ such that: $\left\|d u_{\rho}^{v}\right\|_{L^{\infty}} \leq c^{v},\left\|d \pi(u)_{\rho}\right\|_{L^{\infty}} \leq c^{B}$, and:

$$
\left\|\left(\bar{\partial}_{J_{P}} u_{\rho}\right)^{v}\right\|_{L^{p}} \leq c^{v}|\rho|^{1 / 2 p}, \quad\left\|\bar{\partial}_{J_{B}} \pi(u)_{\rho}\right\|_{L^{p}} \leq c^{B}|\rho|^{1 / 2 p} .
$$

Moreover, by definition of $g_{J_{P}}$ :

$$
\left\|d u_{\rho}\right\|_{L^{p}} \leq\left\|d u_{B, \rho}\right\|_{L^{p}}+\left\|d u_{\rho}^{v}\right\|_{L^{p}}
$$

hence there is a positive uniform constant $c^{P}$ such that:

$$
\left\|d u_{\rho}\right\|_{L^{\infty}} \leq c^{P}, \quad\left\|\bar{\partial}_{J_{P}} u_{\rho}\right\|_{L^{p}} \leq c^{P}|\rho|^{1 / 2 p} .
$$

Also, from (6.4) there is a uniform positive constant $c_{1}^{P}$ such that:

$$
\left\|N_{u_{\rho}}^{P}\left(\xi_{1}\right)-N_{u_{\rho}}^{P}\left(\xi_{2}\right)\right\|_{L^{p}} \leq c_{1}^{P}\left(\left\|\xi_{1}\right\|_{W^{1, p}}+\left\|\xi_{2}\right\|_{W^{1, p}}\right)\left\|\xi_{1}-\xi_{2}\right\|_{W^{1, p}} .
$$

Furthermore, from (6.5), if $u_{t}:=\left\{\left(u_{v t}, u_{v^{\prime} t}\right)\right\}_{v E v^{\prime}}, t \in[0, v)$ is a a path in $\mathcal{B}_{P}^{1, p}$, with $\zeta:=\left.\frac{d}{d t}\right|_{t=0} u_{t}$, and if $u_{\rho, t}$ is the corresponding path of preglued with $\zeta_{\rho}:=\left.\frac{d}{d t}\right|_{t=0} u_{\rho, t}$, there are uniform constants $\widetilde{c}^{P}$ and $\widetilde{c}^{B}$ such that $\widetilde{c}^{B} \leq \widetilde{c}^{P}$ and

$$
\left\|\zeta_{\rho}\right\|_{W^{1, p}} \leq \widetilde{c}^{P}\|\zeta\|_{W^{1, p}},\left\|D_{u_{\rho, 0}}^{P} \zeta_{\rho}\right\|_{L^{p}} \leq\left\|D_{u_{0}}^{P} \zeta\right\|_{L^{p}}+\widetilde{c}^{P}|\rho|^{1 / 2 p}\|\zeta\|_{C^{1}}
$$

and

$$
\begin{aligned}
& \left\|\pi_{*} \zeta_{\rho}\right\|_{W^{1, p}} \leq \widetilde{c}^{B}\left\|\pi_{*} \zeta\right\|_{W^{1, p}}, \\
& \left\|D_{\pi\left(u_{\rho, 0}\right)}^{B} \pi_{*} \zeta_{\rho}\right\|_{L^{p}} \leq\left\|D_{\pi\left(u_{0}\right)}^{B} \pi_{*} \zeta\right\|_{L^{p}}+\widetilde{c}^{B}|\rho|^{1 / 2 p}\|\zeta\|_{C^{1}} .
\end{aligned}
$$

6.2.2. Right inverses. We give the description of right inverses for $D_{u}^{P}$, which are induced by right inverses for $D_{u}^{v}$ and right inverses for $D_{\pi(u)}^{B}$. By assumption, $D_{\pi(u)}^{B}$ and $D_{u}^{v}$ are surjective and we can therefore consider their unique $L^{2}$-orthogonal right inverses, $Q_{\pi(u)}^{B}$ and $Q_{u}^{v}$, with respect to $g_{J_{B}}$ and $g_{J}$. Set

$$
Q_{u}^{P}:=\left(\begin{array}{cc}
\left(Q_{\pi(u)}^{B}\right)^{h} & 0 \\
L_{u}^{\prime} & Q_{u}^{v}
\end{array}\right)
$$


From the matrix expression (3.2) for $D_{u}^{P}$, we get

$$
D_{u}^{P} \circ Q_{u}^{P}:=D_{u}^{P} \circ\left(\begin{array}{cc}
\left(Q_{\pi(u)}^{B}\right)^{h} & 0 \\
L_{u}^{\prime} & Q_{u}^{v}
\end{array}\right)=\left(\begin{array}{cc}
I d & 0 \\
L_{u} \circ\left(Q_{\pi(u)}^{B}\right)^{h}+D_{u}^{v} \circ L_{u}^{\prime} & I d
\end{array}\right),
$$

where $L$ is given by (3.3). Thus $Q_{u}^{P}$ is a right inverse, if $L^{\prime}:\left(\mathcal{E}_{P}\right)_{u}^{h} \rightarrow\left(\mathcal{X}_{P}\right)_{u}^{v}$ verifies

$$
L_{u} \circ\left(Q_{\pi(u)}^{B}\right)^{h}+D_{u}^{v} \circ L_{u}^{\prime}=0 .
$$

A natural choice for $L^{\prime}$ is $L^{\prime}=-Q^{v} \circ L \circ\left(Q^{B}\right)^{h}$.

Remark 6.3. By definition, $L_{u}$ is bounded. Namely, $\left\|L_{u}\right\| \leq C^{\prime \prime}$ for some constant $C^{\prime \prime}$ depending on $\|J\|_{C^{1}},\|d u\|_{L^{\infty}}$, and $\|R\|_{H}$.

The following is immediate.

Lemma 6.4. Let $Q_{u}^{P}$ be a right inverse for $D_{u}^{P}$ and suppose $Q_{\pi(u)}^{B}$ and $Q_{u}^{v}$ are as above. Then $L_{u}^{\prime}$ is uniquely determined by (6.22) and the requirement that $Q_{u}^{P}$ has for image the $L^{2}$-orthogonal complement of $\operatorname{ker} D_{u}^{P}$. In this case,

$$
L^{\prime}=-Q_{u}^{v} \circ L \circ\left(Q_{\pi(u)}^{B}\right)^{h} .
$$

In particular if $\left\|Q_{\pi(u)}^{B}\right\|<C^{h}$ and $\left\|Q_{u}^{v}\right\|<C^{v}$, for some positive constants $C^{v}$ and $C^{h}$ depending on $\|d u\|_{L^{\infty}}$, then $\left\|L^{\prime}\right\|<C^{h} C^{\prime \prime} C^{v}$.

Remark 6.4. The $L^{2}$-orthogonal complementarity condition is a commodity assumption. The lemma above still holds for different choices of right inverses as long as we ask that the image of $Q^{P}$ is given by the images of $Q^{B}$ and $Q^{v}$.

Let $p>2$, and let $(\mathbf{j}, u) \in \widetilde{\mathcal{M}}_{\mathcal{S}_{P}}(P)$. From Assumption (SR), Lemma 6.2 and Proposition 6.1, we have uniform constants, $c^{B}$ and $c^{v}$, and right inverses $Q_{\pi(u)_{\rho}}^{B}$ and $Q_{u_{\rho}}^{v}$ for $D_{\pi(u)_{\rho}}^{B}$ and $D_{u_{\rho}}^{v}$ such that:

$$
\left\|Q_{\pi(u)_{\rho}}^{B} \eta\right\|_{W^{1, p}} \leq c^{B}\|\eta\|_{L^{p}}, \quad\left\|Q_{u_{\rho}}^{v} \eta\right\|_{W^{1, p}} \leq c^{v}\|\eta\|_{L^{p}} .
$$

Similarly to Proposition 6.1 we have:

Proposition 6.2. There exists a constant $c^{P}$ independent of $\rho$ and right inverse $Q^{P}$ for $D^{P}$ such that for $(u, \mathbf{j}) \in \mathcal{M}_{\mathcal{S}_{P}}(P)$ :

$$
Q_{u_{\rho}}^{P}:=\left(\begin{array}{cc}
\left(Q_{\pi(u)_{\rho}}^{B}\right)^{h} & 0 \\
-Q_{u_{\rho}}^{v} \circ L_{u_{\rho}} \circ\left(Q_{\pi(u)_{\rho}}^{B}\right)^{h} & Q_{u_{\rho}}^{v}
\end{array}\right)
$$

and such that

$$
\left\|Q_{u_{\rho}}^{P} \eta\right\|_{W^{1, p}} \leq c^{P}\|\eta\|_{L^{p}}
$$


Proof. The right inverses $Q_{\pi(u)_{\rho}}^{B}$ and $Q_{u_{\rho}}^{v}$ are obtained from quasi-inverses $R_{\pi(u)_{\rho}}^{B}$ for $D_{\pi(u)_{\rho}}^{B}$, and $R_{u_{\rho}}^{v}$ for $D_{u_{\rho}}^{v}$, constructed as in (6.7). From these we construct a quasi-inverse for $D_{u_{\rho}}^{P}$ :

$$
\begin{aligned}
R_{u_{\rho}}^{P}: \mathcal{E}_{P, u_{\rho}}^{p} \equiv \mathcal{E}_{P, u_{\rho}}^{p, h} \oplus \mathcal{E}_{P, u_{\rho}}^{p, v} \rightarrow \mathcal{X}_{P, u_{\rho}}^{1, p} \equiv \mathcal{X}_{P, u_{\rho}}^{1, p, h} \oplus \mathcal{X}_{P, u_{\rho}}^{1, p, v} \\
\left(\eta^{h}, \eta^{v}\right) \mapsto\left(\left(R_{\pi(u)_{\rho}}^{B} \pi_{*} \eta^{h}\right)^{h}+L_{u_{\rho}}^{R} \eta^{h}, R_{u_{\rho}}^{v} \eta^{v}\right) .
\end{aligned}
$$

In fact,

$$
R_{u_{\rho}}^{P}:=\Gamma \circ Q_{\mathbf{w}_{\rho}}^{P} \circ \Lambda .
$$

where, $\mathbf{w}_{\rho}, \Lambda$ and $\Gamma$, are defined as in the $B=p t$ case. Note that $\Gamma$ and $\Lambda$ preserve the splitting induced by the Hamiltonian connection on $T P$, hence they have the following matrix representation

$$
\Lambda \equiv\left(\begin{array}{cc}
\Lambda^{h} & 0 \\
0 & \Lambda^{v}
\end{array}\right) \quad \Gamma \equiv\left(\begin{array}{cc}
\Gamma^{h} & 0 \\
0 & \Gamma^{v}
\end{array}\right) .
$$

It follows from the matrix form of $Q_{w_{\rho}}^{P}$ that

$$
R_{u_{\rho}}^{P}=\left(\begin{array}{cc}
\Gamma^{h}\left(Q_{\pi\left(\mathbf{w}_{\rho}\right)}^{B}\right)^{h} \Lambda^{h} & 0 \\
-\Gamma^{v} Q_{\mathbf{w}_{\rho}}^{v} L_{\mathbf{w}_{\rho}}\left(Q_{\pi\left(\mathbf{w}_{\rho}\right)}^{B}\right)^{h} \Lambda^{h} & \Gamma^{v} Q_{\mathbf{w}_{\rho}}^{v} \Lambda^{v}
\end{array}\right) \equiv\left(\begin{array}{cc}
R_{u_{\rho}}^{h} & 0 \\
L_{u_{\rho}}^{R} & R_{u_{\rho}}^{v}
\end{array}\right),
$$

we end up with the desired expression for $R_{u_{\rho}}^{P}$. Note that $R^{B} \equiv d \pi \circ R^{h}$. We show that $R^{P}$ is bounded and that $D_{u_{\rho}}^{P} R_{u_{\rho}}^{P}$ is invertible for small enough gluing parameters:

$$
\left\|D_{u_{\rho}}^{P} R_{u_{\rho}}^{P} \eta-\eta\right\|_{L^{p}} \leq \frac{C^{P}}{|\log | \rho \|^{1-1 / p}}\|\eta\|_{L^{p}}, \quad\left\|R_{u_{\rho}}^{P} \eta\right\|_{W^{1, p}} \leq \frac{\widetilde{C}^{P}}{2}\|\eta\|_{L^{p}},
$$

for uniform constants $C^{P}$ and $\widetilde{C}^{P}$. But from Lemma 6.2, there are uniform constants, $C^{B}, \widetilde{C}^{B}, C^{v}$ and $\widetilde{C}^{v}$, such that:

$$
\left\|D_{u_{B, \rho}}^{B} R_{u_{B, \rho}}^{B} \eta-\eta\right\|_{L^{p}} \leq \frac{C^{B}}{|\log | \rho \|^{1-1 / p}}\|\eta\|_{L^{p}}, \quad\left\|R_{u_{B, \rho}}^{B} \eta\right\|_{W^{1, p}} \leq \frac{\widetilde{C}^{B}}{2}\|\eta\|_{L^{p}}
$$

and

$$
\left\|D_{u_{\rho}}^{v} R_{u_{\rho}}^{v} \eta-\eta\right\|_{L^{p}} \leq \frac{C^{v}}{|\log | \rho||^{1-1 / p}}\|\eta\|_{L^{p}}, \quad\left\|R_{u_{\rho}}^{v} \eta\right\|_{W^{1, p}} \leq \frac{\widetilde{C}^{v}}{2}\|\eta\|_{L^{p}}
$$

Set $\xi_{\rho}=R_{u_{\rho}}^{P} \eta$. Suppose without loss of generality that $|V|=2$, and let $V=\left\{v, v^{\prime}\right\}$ with edge $e_{f, f^{\prime}}$. Outside the patched annuli $D_{f}\left(r_{v v^{\prime}}^{1 / 4}\right) \backslash D_{f}\left(r_{v v^{\prime}}^{3 / 4}\right)$, we have that $\xi_{\rho}=\xi=Q_{w_{\rho}}^{P} \eta$ and $u_{\rho}=w_{\rho}$, which implies that $D_{u_{\rho}}^{P} \xi_{\rho}=\eta$. Therefore, the desired estimate for $R_{u_{\rho}}^{P}$ is trivially realized on this part of the curve. Then, it suffices to understand what happens on $D_{f}\left(r_{v v^{\prime}}^{1 / 4}\right) \backslash D_{f}\left(r_{v v^{\prime}}^{1 / 2}\right)$. 
In that region $u_{\rho}$ and $\mathbf{w}_{\rho}$ are constant with value $p_{v v^{\prime}}$. Hence, $D_{u_{\rho}}^{P}, D_{u_{v, \rho}}^{P}$ and $D_{u_{v^{\prime}, \rho}}^{P}$, coincide with the standard Cauchy-Riemann operator:

$$
\left(\begin{array}{cc}
\left(\bar{\partial}_{J_{B}\left(\pi\left(p_{v v^{\prime}}\right)\right)}\right)^{h} & 0 \\
0 & \bar{\partial}_{J_{\pi\left(p_{v v^{\prime}}\right)}}
\end{array}\right) .
$$

Furthermore, $L_{u_{\rho}}^{R}$ must vanish since $L_{\mathbf{w}_{\rho}}$ vanishes pointwise. Then, the result follows from the estimations of $R^{B}$ and $R^{v}$ : the first estimate in (6.26) is obtained by choosing $C^{P} \geq \max \left(C^{B}, C^{v}\right)$, and the second by taking $\widetilde{C}^{P} \geq \max \left(\widetilde{C}^{B}, \widetilde{C}^{v}\right)$. Hence, $D_{u_{\rho}}^{P} R_{u_{\rho}}^{P}$ is invertible for small enough gluing parameters and we set:

$$
Q_{u_{\rho}}^{P}:=R_{u_{\rho}}^{P}\left(D_{u_{\rho}}^{P} R_{u_{\rho}}^{P}\right)^{-1} .
$$

Now $D_{u_{\rho}}^{P} R_{u_{\rho}}^{P}$ is of the following form:

$$
D R:=\left(\begin{array}{cc}
D^{h} R^{h} & 0 \\
L_{D^{P} R^{P}} & D^{v} R^{v}
\end{array}\right),
$$

where

$$
L_{D^{P} R^{P}, u_{\rho}}=L_{u_{\rho}} R_{u_{\rho}}^{h}-D^{v} \Gamma^{v} Q_{w_{\rho}}^{v} L_{w_{\rho}}\left(Q_{\pi(w)_{\rho}}^{B}\right)^{h} \Lambda^{h} .
$$

Furthermore,

$$
\left(D^{P} R^{P}\right)^{-1}:=\left(\begin{array}{cc} 
& \left(D^{h} R^{h}\right)^{-1} \\
L_{\left(D^{P} R^{P}\right)^{-1}}:=\left(D^{v} R^{v}\right)^{-1} L_{D R}\left(D^{h} R^{h}\right)^{-1} & \left(D^{v} R^{v}\right)^{-1}
\end{array}\right) .
$$

Since all the operators involved are lower triangular, we must have that

$$
Q_{u_{\rho}}^{P}:=\left(\begin{array}{cc}
\left(Q_{\pi(u)_{\rho}}^{B}\right)^{h} & 0 \\
L_{u_{\rho}}^{\prime \prime} & Q_{u_{\rho}}^{v}
\end{array}\right) .
$$

We identify $L_{u_{\rho}}^{\prime \prime}$. To simplify notations we will omit the $u_{\rho}$ subscripts and we will set $\left(D^{B}\right)^{h}=D^{h}$. Again, $L_{\mathbf{w}_{\rho}}$ vanishes in the region $D_{f}\left(r_{v v^{\prime}}^{1 / 4}\right) \backslash D_{f}\left(r_{v v^{\prime}}^{3 / 4}\right)$, so that the image of $L_{\mathbf{w}_{\rho}}$ must lie in the image of $\Lambda^{v}$. Now, this latter map is injective, therefore

$$
L^{R}=-R^{v}\left(\Lambda^{v}\right)^{-1} L_{\mathbf{w}_{\rho}}\left(Q_{\pi(\mathbf{w})_{\rho}}^{B}\right)^{h} \Lambda^{h} .
$$

A simple computation then gives:

$$
L^{\prime \prime}=L^{R}\left(D^{h} R^{h}\right)^{-1}+R^{v} L_{\left(D^{P} R^{P}\right)^{-1}}=-Q^{v} L R^{h}\left(D^{h} R^{h}\right)^{-1}=-Q^{v} L\left(Q_{B}\right)^{h} .
$$

Finally, let $\left\{u_{t}\right\}_{t \in[0, v)}$ be a path in $\mathcal{B}_{P}^{1, p}$, where $\zeta:=\left.\frac{d}{d t}\right|_{t=0} u_{t}$. Let $\left\{u_{\rho, t}\right\}_{t \in[0, v)}$ be the corresponding path of preglued curves, with $u_{\rho, 0}=: u_{\rho}$. There are uniform constants $\bar{c}^{B}$ and $\bar{c}^{P}$ such that:

$$
\left\|\left.\frac{d}{d t}\right|_{t=0} Q_{\pi\left(u_{\rho, t}\right)}^{B}\right\| \leq \bar{c}^{B}\left\|\pi_{*} \zeta\right\|_{W^{1, p},}, \quad\left\|\left.\frac{d}{d t}\right|_{t=0} Q_{u_{\rho, t}}^{P}\right\| \leq \bar{c}^{P}\|\zeta\|_{W^{1, p}} .
$$


6.2.3. Gluing stable components. We assume here that both $S_{\pi_{*}}$ and $\bar{\pi}_{p t}^{\mathcal{S}_{P}}$ preserves the tree structure of $\mathcal{S}_{P}$. Then $\bar{\pi}_{p t}^{\mathcal{S}_{P}} \equiv \mathcal{F}_{P}, \bar{\pi}_{p t}^{\mathcal{S}_{B}} \equiv \mathcal{F}_{B}$ and

$$
\bar{\pi}_{\mathcal{S}_{P}}: \mathcal{M}_{\mathcal{S}_{P}}(P) \rightarrow \mathcal{M}_{\mathcal{S}_{B}}(B), \quad(u, \mathbf{j}) \mapsto\left(u_{B}:=\pi(u), \mathbf{j}\right) .
$$

This induces a map between orbibundles:

$$
\overline{\bar{\pi} \mathcal{S}_{P}}: \mathcal{L}_{\mathcal{S}_{P}}:=\mathcal{F}_{P}^{*} \mathcal{L}_{\mathcal{S}} \rightarrow \mathcal{L}_{\mathcal{S}_{B}}:=\mathcal{F}_{B}^{*} \mathcal{L}_{\mathcal{S}} \quad(u, \mathbf{j}, \rho) \mapsto(\pi(u), \mathbf{j}, \rho) .
$$

Let $U_{P} \subset \mathcal{M}_{\mathcal{S}_{P}}(P)$ and $U_{B} \subset \mathcal{M}_{\mathcal{S}_{B}}(B)$ be proper open subsets such that $\bar{\pi}_{\mathcal{S}_{P}}\left(U_{P}\right)=U_{B}$. To simplify the exposition, we assume that the maps involved do not have automorphism. If not so, the gluing maps $G l_{\mathcal{S}_{P}}$ and $G l_{\mathcal{S}_{B}}$ obtained below are actually defined on local uniformizing systems for neighborhoods $W^{P}$ and $W^{B}$ around the stable (holomorphic) maps $(u, \mathbf{j}$ ) and $(\pi(u), \mathbf{j})$, which are compatible with the projection $\bar{\pi}_{\mathcal{S}_{P}}$.

From Theorem 6.3 there exists a positive constant $\epsilon_{B}$, and a diffeomorphism

$$
G l_{\mathcal{S}_{B}}: \mathcal{L}_{\mathcal{S}_{B}, \epsilon_{B}, U_{B}}^{*} \rightarrow \mathcal{M}_{0, l}\left(B, \sigma_{B}\right), \quad\left(u_{B}, \mathbf{j}, \rho\right) \mapsto G l_{\mathcal{S}_{P}}\left(u_{B}, \mathbf{j}, \rho\right) .
$$

Similarly to Theorem 6.3 we have:

Theorem 6.5. For small enough positive constant $\epsilon_{P}$, there is a diffeomorphism:

$$
G l_{\mathcal{S}_{P}}: \mathcal{L}_{\mathcal{S}_{P}, \epsilon_{P}, U_{P}}^{*} \rightarrow \mathcal{M}_{0, l}(P, \sigma), \quad(u, \mathbf{j}, \rho) \mapsto G l_{\mathcal{S}_{P}}(u, \mathbf{j}, \rho),
$$

such that:

$$
\bar{\pi} \circ G l_{\mathcal{S}_{P}}=G l_{\mathcal{S}_{B}} \circ \overline{\bar{\pi}}{\mathcal{\mathcal { S } _ { P }}} .
$$

Proof. First, recall that for $(\pi(u), \mathbf{j}, \rho) \in \mathcal{L}_{\mathcal{S}_{B}, \epsilon_{B}, U_{B}}^{*}$,

$$
G l_{\mathcal{S}_{B}}(\pi(u), \mathbf{j}, \rho)=\left(\mathbf{j}_{\rho}, \exp _{\pi(u)_{\rho}} Q_{\pi(u)_{\rho}}^{B}\left(f^{B}(\pi(u), \mathbf{j}, \rho)\right)\right),
$$

where $f^{B}$ is as in (6.10):

$$
f^{B}(\pi(u), \mathbf{j}, \rho) \in B_{\delta_{B}, \pi(u)_{\rho}}(0) \subset L^{p}\left(\Lambda_{J}^{0,1}\left(S^{2}, \pi(u)_{\rho}^{*} T B\right)\right)
$$

for a positive (uniform) constant $\delta_{B}$ given by the Implicit Function Theorem. From the estimates (6.19), (6.20), (6.25), Implicit Function Theorem applies here, and there are uniform constants $\epsilon_{P}$ and $\delta_{P}$, and a map

$$
f^{P}: \mathcal{L}_{\mathcal{S}_{P}, \epsilon_{P}, U_{P}} \rightarrow B_{\delta_{P}}(0)
$$


such that

$$
f^{P}(u, \mathbf{j}, \rho) \in B_{\delta_{P}, u_{\rho}}(0) \subset L^{p}\left(\Lambda_{J}^{0,1}\left(S^{2}, u_{\rho}^{*} T P\right)\right)
$$

is the unique solution to $\bar{\partial}_{J_{P}}\left(\exp _{u_{\rho}} Q_{u_{\rho}}^{P}\left(f^{P}(u, \mathbf{j}, \rho)\right)\right)=0$. Then the gluing map $G l_{\mathcal{S}_{P}}$ is defined by

$$
G l_{\mathcal{S}_{P}}(u, \mathbf{j}, \rho)=\left(\mathbf{j}_{\rho}, \exp _{u_{\rho}} Q_{u_{\rho}}^{P}\left(f^{P}(u, \mathbf{j}, \rho)\right)\right) .
$$

The proof that this is a local diffeomorphism is verbatim the proof of Theorem 6.3 using the estimates (6.21) and (6.27).

Next we show (6.28). We begin by showing that

$$
d \pi \circ f^{P}=f^{B} \circ \bar{\pi}_{\mathcal{S}_{P}} .
$$

By Lemma 6.3, $\pi\left(u_{\rho}\right)=\pi(u)_{\rho}$. Let $\xi^{P}$ denote $Q_{u_{\rho}}^{P} f^{P}(u, \mathbf{j}, \rho)$. Then,

$$
\pi\left(\exp _{u_{\rho}}\left(\xi^{P}\right)\right)=\exp _{\pi(u)_{\rho}} d \pi\left(\xi^{P}\right) .
$$

Since $\xi^{P}$ is the unique solution to $\bar{\partial}_{J_{P}} \exp _{u_{\rho}}\left(\xi^{P}\right)=0$, we deduce that

$$
\bar{\partial}_{J_{B}} \exp _{\pi(u)_{\rho}} d \pi\left(\xi^{P}\right)=0 .
$$

Moreover,

$$
\xi^{B}:=d \pi\left(\xi^{P}\right)=d \pi\left(Q_{u_{\rho}}^{P} f^{P}(u, \mathbf{j}, \rho)\right)=Q_{\pi(u)_{\rho}}^{B} d \pi\left(f^{P}(u, \mathbf{j}, \rho)\right),
$$

implying that $\xi^{B}$ is in the image of $Q_{u_{B, \rho}}^{B}$, so that $d \pi\left(f^{P}(u, \mathbf{j}, \rho)\right)$ is in the image of $f^{B}$ (by the implicit function theorem and choosing $\delta_{P}$ smaller than $\left.\delta_{B}\right)$. Finally,

$$
\begin{aligned}
\bar{\pi}\left(G l_{\mathcal{S}_{P}}(u, \mathbf{j}, \rho)\right) & =\pi\left(\exp _{u_{\rho}} Q_{u_{\rho}}^{P} f^{P}(u, \mathbf{j}, \rho)\right) \\
& =\exp _{\pi(u)_{\rho}}\left(d \pi\left(Q_{u_{\rho}} f^{P}(u, \mathbf{j}, \rho)\right)\right) \\
& =\exp _{\pi(u)_{\rho}}\left(Q_{\pi(u)_{\rho}}^{B} f^{B}(\pi(u), \mathbf{j}, \rho)\right) \\
& =G l_{\mathcal{S}_{B}}(\pi(u), \mathbf{j}, \rho)
\end{aligned}
$$

6.2.4. Gluing: the unstable case. Let $(u, \mathbf{j}) \in \mathcal{M}_{\mathcal{S}_{P}}(P)$, and let $v \in V_{P}$ be a $\pi_{*}$-unstable component. Again, $|v|>2$ is possible. Generalizing the approach in [3], we describe the notion of balanced component when $|v| \leq 2$.

(1) Balanced maps. First assume $|v|=2$. In this case $u_{v}: \Sigma_{v} \rightarrow P$ can be parameterized by $\mathbb{C P}^{1}$ with special points $0=[1: 0]$ and $\infty=[0: 1]$. Identify $\Sigma_{v} \backslash\{\infty\}$ with $\mathbb{C}$. If $\pi\left(u_{v}\right)$ is non-constant, we say that $u_{v}$ is balanced if it is horizontally balanced, i.e., if $\pi\left(u_{v}\right)$ is balanced in the sense of (6.13). On the other hand, if $\pi\left(u_{v}\right)$ is constant, we say that $u_{v}$ is balanced if it is vertically balanced, i.e., if half the vertical energy $\left(\hbar^{v}\right)$ of $u_{v}$ is contained in the unit disc around 0 .

Next, when $|v|=1, \Sigma_{v}$ can be parameterized by $\mathbb{C P}^{1}$ with special point $\infty=[0: 1]$. Identify $\Sigma_{v} \backslash\{\infty\}$ with $\mathbb{C}$. If $\pi\left(u_{v}\right)$ is not constant, $u_{v}$ is balanced 
if it is horizontally balanced, as in the $|v|=2$ case, and if $u_{v}$ is horizontally centered, i.e., if the center of energy of $\pi\left(u_{v}\right)$ is zero. If $\pi\left(u_{v}\right)$ is constant, we say that $u_{v}$ is balanced if it is vertically balanced as above and if vertically centered, i.e., if the mean value for $\left\|d u_{v}^{v}\right\|_{g_{J_{P}}}^{2}$ is zero.

For $i=1,2$, let $\widetilde{\mathcal{M}}_{0, i}^{b, h}\left(P, \sigma_{v}\right)$, resp. $\widetilde{\mathcal{M}}_{0, i}^{b, v}\left(P, \sigma_{v}\right)$, denote the set of horizontally, resp. vertically, balanced $J_{P}$-holomorphic maps with $i=1$ or 2 marked point representing $\sigma_{v}$. In order to simplify notations, $\widetilde{\mathcal{M}}_{0, i}^{b}\left(P, \sigma_{v}\right)$ will designate the set of (vertically and horizontally) balanced $J_{P}$-holomorphic maps with $i$ marked point. When $\pi_{*} \sigma_{v} \neq 0$, the projection $\pi$ naturally induces a map:

$$
\pi: \widetilde{\mathcal{M}}_{0,1}^{b}\left(P, \sigma_{v}\right)=\widetilde{\mathcal{M}}_{0, i}^{b, h}\left(P, \sigma_{v}\right) \rightarrow \widetilde{\mathcal{M}}_{0, i}^{b}\left(B, \pi_{*} \sigma_{v}\right) .
$$

As in the $B=p t$ case, there is a natural smooth surjective map between the moduli space of holomorphic maps to the moduli space of balanced maps, $u_{v} \mapsto u_{v}^{b}$, where $u_{v}^{b}$ is given by (6.14), and it is not hard to see that

$$
\pi\left(u_{v}\right)^{b}=\pi\left(u_{v}^{b}\right) \quad \text { if } \pi_{*} \sigma_{v} \neq 0 .
$$

We conclude that

$$
\mathcal{M}_{0, i}\left(P, \sigma_{v}\right) \cong \widetilde{\mathcal{M}}_{0, i}^{b}\left(P, \sigma_{v}\right) / S^{1}, \quad i=1,2,
$$

and when $\pi_{*} \sigma \neq 0$, the map $\pi$ in (6.29) descends to the (expected) map:

$$
\bar{\pi}: \mathcal{M}_{0, i}\left(P, \sigma_{v}\right) \rightarrow \mathcal{M}_{0, i}\left(B, \pi_{*} \sigma_{v}\right), \quad i=1,2 .
$$

Finally, we say that $(u, \mathbf{j}) \in \widetilde{\mathcal{M}}_{\mathcal{S}_{P}}(P)$ is balanced if each of its $\pi_{*}$-unstable components $u_{v}$ with $|v| \leq 2$ is balanced. The corresponding moduli space of balanced $J_{P}$-holomorphic maps is denoted $\widetilde{\mathcal{M}}_{\mathcal{S}_{P}}^{b}(P)$. Similarly to $(6.16)$, we have a natural identification,

$$
\mathcal{M}_{\mathcal{S}_{P}}(P)=\widetilde{\mathcal{M}}_{\mathcal{S}_{P}}^{b}(P) / \mathrm{Aut}_{\mathrm{red}}^{P}
$$

where Aut $\mathrm{t}_{\text {red }}^{P}$ denotes the group of reparameterizations acting on $\widetilde{\mathcal{M}}_{\mathcal{S}_{P}}^{b}(P)$ (see $(6.15))$. Observe that the projection $\pi_{\mathcal{S}_{P}}$ restricts to an $\mathrm{Aut}_{\text {red }}^{P}$, Aut red equivariant map

$$
\pi_{\mathcal{S}_{P}}: \widetilde{\mathcal{M}}_{\mathcal{S}_{P}}^{b}(P) \rightarrow \widetilde{\mathcal{M}}_{\mathcal{S}_{B}}^{b}(B)
$$

and descends to $\bar{\pi}_{\mathcal{S}_{P}}: \mathcal{M}_{\mathcal{S}_{P}}(P) \rightarrow \mathcal{M}_{\mathcal{S}_{B}}(B)$ after quotienting by the reparameterizations.

Remark 6.5. Let $P$ denote the bundle $\left(S^{2} \times S^{2}, \omega_{0}+\omega_{0}\right)$ over the base $B:=\left(S^{2}, \omega_{0}\right)$, where $\omega_{0}$ denotes the Fubini-Study form on $S^{2}$. Here, $\pi$ represents the projection to the first factor, $J_{P}$ is the product complex structure. Consider the holomorphic section $u(z)=(z, z+b)$. A simple computation shows that $\pi(u)$ is balanced (the map $z \mapsto a z+b$ is balanced if and only 
if $|a|=1$ and $b=0)$. In addition, one sees that $u(z-b)$ is vertically balanced. Note that if we had adopted the definition for balanced maps in $P$ with respect to the energy density $\|d u\|_{g_{J_{P}}}^{2}$, the mapping (6.29) would not necessarily exist. Indeed, the map $u(z-b / 2)$ is balanced in this sense, but projects to $z-b / 2$ which is not balanced.

Finally, had we defined a balanced map in $P$ as being both horizontally and vertically balanced, the compatibility (6.30) may not be realized. For example, consider the maps $u(z)=(z, a z)$, for $a \in \mathbb{R}^{+}$, with $a \neq 1$. Then the center of energy of $u$ is 0 , so that it is horizontally balanced. However, the map is not vertically balanced, and if $\hbar^{h}$ denotes the energy of the projection $\pi(u)$, then the energy of $u$ in the unit disc around 0 is given by $\hbar^{h}+\pi\left(1-\frac{1}{1+a^{2}}\right)$, which gives $\hbar$ if and only if $a=1$.

(2) Gluing of balanced maps and compatibility. Let $\mathcal{S}_{B}^{u}$ and $\mathcal{S}^{u}$ respectively denote the projections of $\mathcal{S}_{P}$ under $\pi_{*}$ and $\mathcal{F}_{P}$. These stratum data are not stable here. Note that $\mathcal{S}_{B}^{u}$ and $\mathcal{S}_{P}$ have the same tree structure but the homological data for $\mathcal{S}_{B}^{u}$ are the projection of the homological data for $\mathcal{S}_{P}$ under $\pi_{*}$.

Let $\widetilde{\mathcal{L}}_{\mathcal{S}_{P}}$ and $\widetilde{\mathcal{L}}_{\mathcal{S}_{B}}$ denote the bundles of gluing parameters over the balanced moduli spaces $\widetilde{\mathcal{M}}_{\mathcal{S}_{P}}(P)$ and $\widetilde{\mathcal{M}}_{\mathcal{S}_{B}}(B)$ obtained by pull-backing $\widetilde{\mathcal{L}}_{\mathcal{S}^{u}}$ under $\mathcal{F}_{P}$ and $\mathcal{F}_{B}$, respectively. Consider the bundle map lifting $\bar{\pi}_{S_{P}}$ :

$$
\overline{\bar{\pi}}{\mathcal{\mathcal { S } _ { P }}}: \widetilde{\mathcal{L}}_{\mathcal{S}_{P}} \rightarrow \widetilde{\mathcal{L}}_{\mathcal{S}_{B}}, \quad(u, \mathbf{j}, \rho) \mapsto\left(\bar{\pi}_{\mathcal{S}_{P}}(u, \mathbf{j}), \rho^{\mathrm{st}}\right),
$$

where $\rho$ st denotes the stabilization of $\rho$ with respect to $\pi_{*}$ this time (see (6.17)). This map descends to a well-defined map:

$$
\overline{\bar{\pi}} \mathcal{S}_{P}: \mathcal{L}_{\mathcal{S}_{P}}:=\widetilde{\mathcal{L}}_{\mathcal{S}_{P}} / \mathrm{Aut}_{\mathrm{red}}^{P} \rightarrow \mathcal{L}_{\mathcal{S}_{B}}:=\widetilde{\mathcal{L}}_{\mathcal{S}_{B}} / \mathrm{Aut}_{\text {red }}^{B}
$$

Let $U_{P} \subset \mathcal{M}_{\mathcal{S}_{P}}(P)$ and $U_{B} \subset \mathcal{M}_{\mathcal{S}_{B}}(B)$ be proper open subsets such that $\bar{\pi}_{\mathcal{S}_{P}}\left(U_{P}\right)=U_{B}$. From the discussion in Section 6.1.3, we have a gluing map,

$$
G l_{\mathcal{S}_{P}}^{b}: \widetilde{\mathcal{L}}_{\mathcal{S}_{P}, \epsilon_{P}, U_{P}}^{*} / \mathrm{Aut}_{\text {red }}^{P} \rightarrow \mathcal{M}_{0, l}(P, \sigma),
$$

and a gluing map $G l_{\mathcal{S}_{B}}^{b}$ above $U_{B}$ defined similarly. We prove the following generalization of Theorem 6.4:

Theorem 6.6. The gluing maps $G l_{\mathcal{S}_{P}}^{b}$ and $G l_{\mathcal{S}_{B}}^{b}$ are local diffeomorphisms such that

$$
\bar{\pi} \circ G l_{\mathcal{S}_{P}}^{b}=G l_{\mathcal{S}_{B}}^{b} \circ \overline{\bar{\pi}}{\overline{\mathcal{S}_{P}}} .
$$

Proof. The diffeomorphism issue follows directly from Theorem 6.4. The compatibility (6.31) is obtained as follows. Let $\widetilde{\mathcal{M}}_{\mathcal{S}_{B}^{u}}^{b}(B)$ denote the image of $\widetilde{\mathcal{M}}_{\mathcal{S}_{P}}^{b}(P)$ under $\pi:(u, \mathbf{j}) \mapsto(\pi(u), \mathbf{j})$. Note that the parameterization of the domain $\pi(u)$ comes from the parameterization of the domain of $u$, which 
is fixed up to an $S^{1}$-action. Now, let $\widetilde{\mathcal{L}}_{\mathcal{S}_{B}^{u}}^{b}$ denote the pull-back of $\widetilde{\mathcal{L}}_{\mathcal{S}^{u}}$ under the forgetting-the-map map. Then, by assumption (SR), we can construct a gluing map $\widetilde{G l}_{\mathcal{S}_{B}^{u}}^{b}$ with value in $\widetilde{\mathcal{M}}_{0, l}\left(B, J_{B}\right)$, and domain a neighborhood of the zero section in $\widetilde{\mathcal{L}}_{\mathcal{S}_{B}^{u}}$ restricted to $\pi\left(U_{P}\right)$. This map is equivariant with respect to the balanced maps automorphisms, but since $\mathcal{S}_{B}^{u}$ is not stable, it is not a local diffeomorphism nor injective. Nevertheless, $\widetilde{G l}_{\mathcal{S}_{B}^{u}}^{b}$ still verifies the compatibility:

$$
\pi \circ \widetilde{G l}_{\mathcal{S}_{P}}^{b}=\widetilde{G l}_{\mathcal{S}_{B}^{u}}^{b} \circ \pi
$$

Again, the problem is localized in the contracted branches and the connecting branches.

Contracted branch. Set $\left(u_{B}, \mathbf{j}_{B}\right):=\pi_{\mathcal{S}_{P}}(u, \mathbf{j})$. As in Theorem (6.4), for every $\rho$ the glued surface $\mathbf{j}_{\rho}$ is isomorphic to $\mathbf{j}_{B}$, i.e., to the domain of the $\pi_{*}$-stable component. Therefore, $u_{B}$ and $\widetilde{G l}_{\mathcal{S}_{P}}^{b}(u, \mathbf{j}, \rho)$ have the same domain. Fix $\rho_{0}$ with small radius. We show that

$$
\widetilde{G l}_{\mathcal{S}_{B}^{u}}^{b}\left(\pi(u), \mathbf{j}, \rho_{0}\right)=\left(u_{B}, \mathbf{j}_{B}\right) .
$$

By definition, the pregluing of $\left(\pi(u), \mathbf{j}, \rho_{0}\right)$ coincides with $\pi(u)$ except on disc with radius determined by $\rho_{0}$ on which

$$
\pi(u)_{\rho_{0}}(z) \equiv \operatorname{pgl}_{B}\left(\pi(u), \mathbf{j}, \rho_{0}\right)(z)=\exp _{\pi\left(p_{\infty}\right)}\left(\beta\left(z / \rho_{0}^{1 / 4}\right) \exp _{\pi\left(p_{\infty}\right)}^{-1}\left(\pi\left(u_{0}(z)\right)\right),\right.
$$

$p_{\infty}$ being the image under $u$ of the point of the root identified to $\infty$. Set

$$
\xi:=\exp _{\pi\left(p_{\infty}\right)}^{-1}\left(\pi\left(u_{0}(z)\right)\right) \quad \text { and } \quad \beta_{\rho_{0}}:=\beta\left(z / \rho_{0}^{1 / 4}\right) .
$$

Then, $\widetilde{G l}_{\mathcal{S}_{B}^{u}}\left(\pi(u), \mathbf{j}, \rho_{0}\right)$ coincides with $u_{B} \equiv \exp _{\pi(u)_{\rho_{0}}}\left(\left(1-\beta_{\rho_{0}}\right) \xi\right)$.

Connecting branch. As in Theorem (6.4), we can assume the contracting branch is a connecting chain. By adding $k$-marked point, one for each component of the connecting branch, $\pi(u)$ becomes stable. Let $\mathbf{j}^{\prime}$ denote the conformal structure resulting from this operation. Let $\widetilde{\mathcal{M}}_{\mathcal{S}_{B}^{u}(k)}^{b}(B)$ denote the stabilization by adding $k$-marked points. We have a gluing map

$$
\widetilde{G l}_{\mathcal{S}_{B}^{u}(k)}: \widetilde{\mathcal{L}}_{\mathcal{S}_{B}^{u}(k)}^{*} \equiv \mathcal{F}_{B}^{*} \widetilde{\mathcal{L}}_{\mathcal{F}_{B}\left(\mathcal{S}_{B}^{u}(k)\right)} \rightarrow \widetilde{\mathcal{M}}_{0, l+k}\left(B, \sigma_{B}\right) .
$$

By definition of the gluing, and since the components of $\pi(u)$ coming from the connecting branch are constant, the maps

$$
\widetilde{G l}_{\mathcal{S}_{B}^{u}(k)}\left(\pi(u), \mathbf{j}^{\prime}, \rho\right) \quad \text { and } \quad \widetilde{G l}_{\mathcal{S}_{B}^{u}}^{b}(\pi(u), \mathbf{j}, \rho)
$$

coincide for every $\rho$ and any choice of balanced parameterization on the domain $\Sigma$ of $\mathbf{j}$ (here the Aut $(\mathbf{j})$-equivariance of $G l_{\mathcal{S}_{B}^{u}}$ is needed). Note that $\mathbf{j}_{\rho}$ is obtained from $\mathbf{j}_{\rho}^{\prime}$ by forgetting the added marked points. We compare the gluing of $\left(\pi(u), \mathbf{j}^{\prime}\right)$ with that of $\left(u_{B}, \mathbf{j}_{B}\right):=\pi_{\mathcal{S}_{P}}(u, \mathbf{j})$. Note that $\left(u_{B}, \mathbf{j}_{B}\right)$ 
is the image of $\left(\pi(u), \mathbf{j}^{\prime}\right)$ via the map that forgets the added marked points and stabilizes. The fiber over $\left(u_{B}, \mathbf{j}_{B}\right)$ corresponds to the set of all possible reparameterizations for $\mathbf{j}^{\prime}$, since $u_{B}$ and $\pi(u)$ have same image. Thus, it suffices to identify the gluings of the corresponding domains but this follows from Theorem 6.4. Finally,

$$
\begin{aligned}
\pi \circ \widetilde{G l}_{\mathcal{S}_{P}}(u, \mathbf{j}, \rho) & =\widetilde{G l}_{\mathcal{S}_{B}^{u}}(\pi(u), \mathbf{j}, \rho) \\
& =\widetilde{G l} l_{\mathcal{S}_{B}^{u}(k)}\left(\pi(u), \mathbf{j}^{\prime}, \rho\right)=\widetilde{G l}_{\mathcal{S}_{B}}\left(u_{B}, \mathbf{j} B, \rho^{\mathrm{st}}\right) \\
& =\widetilde{G l} l_{\mathcal{S}_{B}} \circ \overline{\bar{\pi}}{\overline{\mathcal{S}_{P}}}(u, \mathbf{j}, \rho) .
\end{aligned}
$$

From now on, we will always assume that gluing maps are obtained by considering balanced maps, and we will drop the $b$ indices from the notations for the gluing maps.

6.2.5. Gluing maps between strata. One can generalize the preceding discussions and introduce gluing maps between different stable strata. Let $\mathcal{S}_{X}$ and $\mathcal{S}_{X}^{\prime}$ be stable stratum data for $\overline{\mathcal{M}}_{0, l}(X, A)$, such that $\mathcal{S}_{X} \prec \mathcal{S}_{X}^{\prime}$. Let $\mathcal{S}$ and $\mathcal{S}^{\prime}$ denote their projections under $\mathcal{F}_{X}$, and consider the bundles $\mathcal{L}_{\mathcal{S}, \mathcal{S}^{\prime}}$ and $\mathcal{L}_{\mathcal{S}_{X}, \mathcal{S}_{X}^{\prime}}:=\mathcal{F}_{X}^{*} \mathcal{L}_{\mathcal{S}, \mathcal{S}^{\prime}}$. For a proper open subset $U_{X}$ of $\mathcal{M}_{\mathcal{S}_{X}}(X)$ there exists a positive constant $\epsilon_{X}$ and a diffeomorphism:

$$
G l_{\mathcal{S}_{X}, \mathcal{S}_{X}^{\prime}}: \mathcal{L}_{\mathcal{S}_{X}, \mathcal{S}_{X}^{\prime}, \epsilon_{X}, U_{X}}^{*} \rightarrow \mathcal{M}_{\mathcal{S}_{X}^{\prime}}(X),
$$

which coincides with the identity on the zero section. Also, from the definition of $\mathcal{L}_{\mathcal{S}_{X}, \mathcal{S}_{X}^{\prime}}$, a point of $\mathcal{L}_{\mathcal{S}_{X}}$ is locally given by a tuple $\left(u, \mathbf{j}, \rho_{1}, \rho_{2}\right)$ where $\left(u, \mathbf{j}, \rho_{1}\right) \in \mathcal{L}_{\mathcal{S}_{X}, \mathcal{S}_{X}^{\prime}}$ and where $\rho_{2}$ accounts for the remaining gluing parameters. Therefore, $G l_{\mathcal{S}_{X}, \mathcal{S}_{X}^{\prime}}$ induces a map

$$
\mathcal{L}_{\mathcal{S}_{X}} \rightarrow \mathcal{L}_{\mathcal{S}_{X}^{\prime}}, \quad\left(u, \mathbf{j}, \rho_{1}, \rho_{2}\right) \mapsto\left(G l_{\mathcal{S}_{X}, \mathcal{S}_{X}^{\prime}}\left(u, \mathbf{j}, \rho_{1}\right), \rho_{2}\right) .
$$

It follows that $\mathcal{L}_{\mathcal{S}_{X}}$ coincides with the pullbacks $G l_{\mathcal{S}_{X}, \mathcal{S}_{X}^{\prime}}^{*} \mathcal{L}_{\mathcal{S}_{X}^{\prime}}$. Suppose now we have a third stratum data $\mathcal{S}_{X}^{\prime \prime}$ such that $\mathcal{S}_{X}^{\prime} \prec \mathcal{S}_{X}^{\prime \prime}$. Since $G l_{\mathcal{S}_{X}, \mathcal{S}_{X}^{\prime}}$ is a local diffeomorphism, we can define a new gluing map:

$$
G l_{\mathcal{S}_{X}^{\prime}, \mathcal{S}_{X}^{\prime \prime}}^{\prime}:=G l_{\mathcal{S}_{X}, \mathcal{S}_{X}^{\prime \prime}} \circ G l_{\mathcal{S}_{X}, \mathcal{S}_{X}^{\prime}}^{-1}: \mathcal{L}_{\mathcal{S}_{X}^{\prime}, \mathcal{S}_{X}^{\prime \prime}, \epsilon_{X}, \operatorname{Im}\left(G l_{\mathcal{S}_{X}, \mathcal{S}_{X}^{\prime}}\right)} \rightarrow \mathcal{M}_{\mathcal{S}_{X}^{\prime}}(X),
$$

extending the identity map on the zero section. This new gluing does not necessarily coincide with $G l_{\mathcal{S}_{X}^{\prime}, \mathcal{S}_{X}^{\prime \prime}}$. The equality would mean that the gluing procedure is associative, which is a priori not true due to the numerous choices made along the gluing construction (in particular the independence with respect to the choice of right inverses). Nevertheless we can see that these maps are close, in the $C^{\infty}$ sense, which is enough to give the moduli spaces the structure of smooth orbifolds, as we will see in the next Section.

Now, consider the Hamiltonian Fibration case $\pi: P \rightarrow B$. Let $\mathcal{S}_{P}$ and $\mathcal{S}_{P}^{\prime}$ be stable stratum data for $\overline{\mathcal{M}}_{0, l}(P, \sigma)$ such that $\mathcal{S}_{P} \prec \mathcal{S}_{P}^{\prime}$, and let $\mathcal{S}_{B}$ and 
$\mathcal{S}_{B}^{\prime}$ be their corresponding projections via $S_{\pi_{*}}$. We see that $\mathcal{S}_{B} \prec \mathcal{S}_{B}^{\prime}$. By the discussion above, for $U_{P}$ and $U_{B}$ proper open subsets of $\mathcal{M}_{\mathcal{S}_{P}}(P)$ and $\mathcal{M}_{\mathcal{S}_{B}}(B)$ such that $\bar{\pi}_{\mathcal{S}_{P}}\left(U_{P}\right)=U_{B}$, we do have gluing maps $G l_{\mathcal{S}_{B}, \mathcal{S}_{B}^{\prime}}$ and $G l_{\mathcal{S}_{P}, \mathcal{S}_{P}^{\prime}}$ such that:

$$
\left.G l_{\mathcal{S}_{B}, \mathcal{S}_{B}^{\prime}} \circ \overline{\bar{\pi}_{\mathcal{S}_{P}}}\right|_{\mathcal{L}_{\mathcal{S}_{P}, \mathcal{S}_{P}^{\prime}}}=\bar{\pi}_{\mathcal{S}_{P}^{\prime}} \circ G l_{\mathcal{S}_{P}, \mathcal{S}_{P}^{\prime}}
$$

Suppose now we have a third stratum data $\mathcal{S}_{P}^{\prime \prime}$ projecting on $\mathcal{S}_{B}^{\prime \prime}$ and such that $\mathcal{S}_{P}^{\prime} \prec \mathcal{S}_{P}^{\prime \prime}$. Then we also have the commutativity:

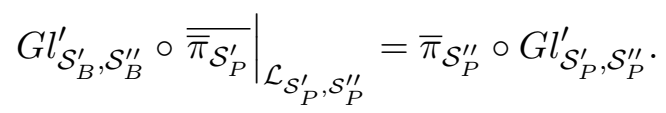

6.3. Fibration of moduli spaces: proof of Theorem C. Following [3], Section 13.2, we show below how the compactified moduli spaces can be given the structure of a differentiable orbifolds compatibly with the $\bar{\pi}_{\mathcal{S}_{P}}$. As observed at the beginning of Section 3, this will imply Theorem 6.1.

6.3.1. Charts data and admissible gluing maps. Consider a Fredholm system $(\mathcal{B}, \mathcal{E}, s)$ with moduli space $\mathcal{M}=s^{-1}(0)$, modeled on maps. Assume that the linearization $L_{x_{0}}$ at $x_{0} \in \mathcal{M}$ is surjective. Then, a standard construction gives a local coordinate chart around $x_{0}$. Such a chart is given by a triple $(U, \phi, f)$ where:

(i) $U$ is a submanifold of a neighborhood $V_{x_{0}}$ of zero in $T_{x_{0}} \mathcal{B}$ (which we identify to a neighborhood $V_{x_{0}}$ of $x_{0}$ in $\mathcal{B}$ via the exponential map);

(ii) $\phi: U \times B_{\delta} \rightarrow V_{x_{0}}^{\prime}$ is a diffeomorphism where $B_{\delta} \subset \mathcal{E}_{x_{0}}$ is an open ball, $V_{x_{0}}^{\prime}$ is a neighborhood of 0 in $V_{x_{0}}$;

(iii) $f$ is a smooth section $f: U \rightarrow B_{\delta}$;

with the property that

$$
\Psi: U \stackrel{1 \times f}{\longrightarrow} U \times B_{\delta} \stackrel{\phi}{\longrightarrow} V_{x_{0}}^{\prime}
$$

is a diffeomorphism from $U$ onto $V_{x_{0}}^{\prime} \cap \mathcal{M}$. Here $x_{0}$ serves as a reference point. More generally, fix $x_{0} \in \mathcal{B}$, which may not belong to $\mathcal{M}$, and let $V_{x_{0}} \subset \mathcal{B}$ be a neighborhood of $x_{0}$.

Definition 6.1. A triple $(U, \phi, \Psi)$, or $(U, \phi, f)$, verifying conditions (i), (ii) and (iii) above, is a called chart data for $\mathcal{M}$.

Remark 6.6. It immediately follows from the definition that the triples $\left(U_{P}, f^{P}, G l_{\mathcal{S}_{P}}\right)$ and $\left(U_{B}, f^{B}, G l_{\mathcal{S}_{B}}\right)$ give charts data for both $\mathcal{M}_{0, l}(P)$ and $\mathcal{M}_{0, l}(B)$. Moreover, these charts are compatible with $\bar{\pi}$.

In fact, one can construct chart data for $\mathcal{M}_{\mathcal{S}_{P}}(P)$ from pairs, $\mathcal{Q}_{P}:=$ $\left(U_{P}, Q^{P}\right)$, where $U_{P}$ is a smooth submanifold of $\mathcal{B}_{\mathcal{S}_{P}}^{1, p}$, and where

$$
Q^{P}:=\left\{Q_{u}^{P} \mid u \in U_{P}\right\}
$$


is a smooth $U_{P}$-family of right inverses for $D_{u}^{P}$. In order to do so, we assume the following conditions (that we actually met when constructing the gluing maps):

\section{Assumption 6.1.}

- for every $u \in U_{P}$,

$$
\|d u\|_{L^{p}} \leq C_{P} \quad \text { and } \quad\left\|\bar{\partial}_{J_{P}} u\right\|_{L^{p}} \leq \epsilon_{P}
$$

- for all $\xi \in T_{u} U_{P}$,

$$
\left\|\frac{d}{d \xi} \bar{\partial}_{J_{P}} u\right\|_{L^{p}} \leq \epsilon_{P}\|\xi\|_{W^{1, p}},
$$

- the family $Q_{u}^{P}$ is Lipschitz continuous for the constant $C_{P}$ and for all $u \in U_{P}$ :

$$
\left\|Q_{u}^{P}\right\| \leq C_{P}
$$

the constants $C_{P}$ and $\epsilon_{P}$ being such that $C_{P} \epsilon_{P} \ll 1$, with $\epsilon_{P}$ small.

The chart data are then given as follows. Fix $u_{0} \in U_{P}$, and let $W$ be a neighborhood of 0 in $T_{u_{0}} \mathcal{B}_{\mathcal{S}_{P}}^{1, p}$. Denote by $U_{P}$, the lift (around $u_{0}$ ) of $U_{P}$ in $W$ via $\exp _{u_{0}}^{-1}$. Then set

$$
\phi_{P}: U_{P} \times \mathcal{E}_{P, u_{0}}^{p}\left(\mathcal{S}_{P}\right) \rightarrow \mathcal{X}_{P, u_{0}}^{1, p}, \quad(\xi, \eta) \mapsto \xi+Q_{u_{0}}^{P} \eta .
$$

From the assumptions above, there is a unique smooth map $f^{P}: U_{P} \rightarrow B_{\delta}$, around $u_{0}$, such that

$$
\bar{\partial}_{J_{P}} \exp _{u_{0}} \phi_{P}\left(\xi, f^{P}(\xi)\right)=0 .
$$

By reducing $\delta$ and the neighborhoods involved, we further have that $\phi_{P}$ is diffeomorphic, hence $\left(U_{P}, \phi_{P}, f^{P}\right)$ is a chart data for $\mathcal{M}_{\mathcal{S}_{P}}(P)$.

Remark 6.7. Regarding compatibility of the coordinate charts one needs to be careful, as pointed out in Section $3[\mathbf{2 0}]$. In fact, the $C^{\infty}$ compatibility is ensured if we restrict our attention to smooth stable maps, which is sufficient to study pseudo-holomorphic stable maps (by elliptic regularity).

From the data $\mathcal{Q}_{P}$, we can furthermore define another type of gluing map. We explain this. Suppose that $\left(U_{P}, \phi_{P}, f^{P}\right)$ is a chart data for a proper subset $\widetilde{U}_{P}$ of $\mathcal{M}_{\mathcal{S}_{P}}(P)$, i.e., $\widetilde{U}_{P}$ is the image of the diffeomorphism: $\Psi_{P}:=$ $\phi_{P} \circ\left(1 \times f^{P}\right)$. By further reducing $U_{P}$ if necessary, we can find a pair

$$
\mathcal{Q}_{P}^{\prime}=\left(U_{P}^{\prime}:=\operatorname{pgl}\left(\Psi_{P}^{-1}\left(\widetilde{U}_{P}\right)\right), Q^{\prime}\right)
$$

where $Q^{\prime}$ is a family of right inverses for the elements in $U_{P}^{\prime}$ constructed from the original family of right inverses $Q^{P}$. These data provide a new gluing map defined by the composition:

$$
G l_{\mathcal{Q}_{P}}:\left.\left.\mathcal{L}_{\mathcal{S}_{P}}^{*}\right|_{\widetilde{U}_{P}} \longrightarrow \Psi_{P}^{*} \mathcal{L}_{\mathcal{S}_{P}}^{*}\right|_{U_{P}} \stackrel{G l}{\longrightarrow} \mathcal{M}_{0, l}(P, \sigma) .
$$


Following the terminology used in $[\mathbf{3}]$ (Definition 11.1), we say that $G l_{\mathcal{Q}_{P}}$ is admissible. Moreover, if $U_{P} \subset \mathcal{M}_{\mathcal{S}_{P}}(P)$, we say that $G l_{\mathcal{Q}_{P}}$ is of type-1, otherwise we say that it is of type-2. In particular, the gluing map $G l_{\mathcal{S}_{P}}$ constructed directly from $\widetilde{U}_{P}$ is admissible and of type-1.

Using gluing maps we introduce a topology basis on $\overline{\mathcal{M}}_{0, l}(P, \sigma)$ : an open neighborhood of $(u, \mathbf{j}) \in \overline{\mathcal{M}}_{0, l}(P, \sigma)$ will be the image of some gluing map $G l_{\mathcal{S}_{P}}$ previously constructed. Hence, a neighborhood is given by charts data of the type $\left(\mathcal{L}_{\mathcal{S}_{P}, \epsilon_{P}, U_{P}}, G l_{\mathcal{S}_{P}}\right)$. By a standard argument these charts are $C^{0}$ compatible ([3] Theorem 11.2), hence they indeed define a topology basis on $\overline{\mathcal{M}}_{0, l}(P, \sigma)$.

That these charts are $C^{0}$ compatible is proved by comparing any admissible gluing map $G l_{\mathcal{Q}_{P}}$ arising from a chart data $\mathcal{Q}_{P}$ for a proper subset $\widetilde{U}_{P} \subset \mathcal{M}_{\mathcal{S}_{P}}(P)$, with the type-1 gluing map $G l_{\mathcal{S}_{P}}$ on $\widetilde{U}_{P}$. Concretely one shows that for small enough $\rho$, the map $\left(G l_{\mathcal{S}_{P}}\right)^{-1} G l_{\mathcal{Q}_{P}}$ is close to the identity map, hence continuous. Thus, the moduli space $\overline{\mathcal{M}}_{0, l}(P, \sigma)$ has the structure of an orbifold in the topology given by the gluing maps.

The differentiable $\left(C^{1}\right)$ orbifold structure is given by the two lemmas in the next subsection. In these two lemmas we prove more. Namely, we construct differentiable atlases on $\overline{\mathcal{M}}_{0, l}(P, \sigma)$ and $\overline{\mathcal{M}}_{0, l}\left(B, \sigma_{B}\right)$ compatibly with $\bar{\pi}$.

6.3.2. Structure of orbi-bundle. Consider the fibration context. We begin by the following observation. Let $\mathcal{Q}_{P}:=\left(U_{P}, Q^{P}\right)$ projecting onto $\mathcal{Q}_{B}:=\left(U_{B}, Q^{B}\right)$ in the sense that $\bar{\pi}_{\mathcal{S}_{P}}\left(U_{P}\right)=U_{B}$ and $Q^{P}$ is of the matrix form (6.22). Also, suppose that both pairs satisfy the assumption (6.1), and that they generate charts data, $\left(U_{P}, \phi_{P}, f^{P}\right)$ and $\left(U_{B}, \phi_{B}, f^{B}\right)$, for some proper open subsets, $\widetilde{U}_{P} \subset \mathcal{M}_{\mathcal{S}_{P}}(P)$ and $\widetilde{U}_{B} \subset \mathcal{M}_{\mathcal{S}_{B}}(B)$, such that $\bar{\pi}_{\mathcal{S}_{P}}\left(\widetilde{U}_{P}\right)=\widetilde{U}_{B}$. Then, repeating the arguments in the gluing map section we obtain that

$$
\bar{\pi} \circ G l_{\mathcal{Q}_{P}}=G l_{\mathcal{Q}_{B}} \circ \overline{\bar{\pi}}{\mathcal{\mathcal { S } _ { P }}} .
$$

This implies that the orbifold structures on the compactified moduli spaces are defined compatibly with $\bar{\pi}$, which is continuous, open and surjective, in the topology of the gluing maps. We now construct $C^{1}$ atlases on both $\overline{\mathcal{M}}_{0, l}(P, \sigma)$ and $\overline{\mathcal{M}}_{0, l}\left(B, \sigma_{B}\right)$ compatibly with $\bar{\pi}$. In order to do so we introduce stratum-coverings.

Definition 6.2 ([3], Definition 13.2). A strata-covering of $\overline{\mathcal{M}}_{0, l}(P, \sigma)$ consists in pairs $\left(U_{P}, \epsilon_{\mathcal{S}_{P}}\right)$ for each stratum data $\mathcal{S}_{P}$ such that:

- $U_{P}$ is a proper open subset in $\mathcal{M}_{\mathcal{S}_{P}}(P)$,

- there exists a well-defined gluing map $G l_{\mathcal{S}_{P}}$ with domain $\mathcal{L}_{\mathcal{S}_{P}, \epsilon_{\mathcal{S}_{P}}, U_{P}}$, 
- letting $W_{\mathcal{S}_{P}}$ be the image $G l_{\mathcal{S}_{P}}\left(\mathcal{L}_{\mathcal{S}_{P}, \epsilon_{\mathcal{S}_{P}}, U_{P}}\right)$, we have that for any two (effective) stratum data $\mathcal{S}_{P}$ and $\mathcal{S}_{P}^{\prime}$ :

$$
W_{\mathcal{S}_{P}} \cap W_{\mathcal{S}_{P}^{\prime}} \neq \emptyset \quad \text { iff } \quad \mathcal{S}_{P} \prec \mathcal{S}_{P}^{\prime}, \quad \text { or } \quad \mathcal{S}_{P}^{\prime} \prec \mathcal{S}_{P}
$$

- the family $\left\{W_{\mathcal{S}_{P}}\right\}_{\mathcal{D}_{0, l}^{\sigma, J_{P}}}$ yields an open covering of $\overline{\mathcal{M}}_{0, l}(P, \sigma)$.

Lemma 6.5. There exists strata-coverings $\left(U_{P}, \epsilon_{\mathcal{S}_{P}}\right)$ and $\left(U_{B}, \epsilon_{\mathcal{S}_{B}}\right)$ for $\overline{\mathcal{M}}_{0, l}(P, \sigma)$ and $\overline{\mathcal{M}}_{0, l}\left(B, \sigma_{B}\right)$, such that $\bar{\pi}_{\mathcal{S}_{P}}\left(U_{P}\right)=U_{B}$.

Proof. The proof is an induction on the stratum data in $\mathcal{D}^{B}:=\mathcal{D}_{0, l}^{\sigma_{B}, J_{B}}$ and $\mathcal{D}^{P}:=\mathcal{D}_{0, l}^{\sigma, J_{P}}$. Let $\mathcal{S}_{B, 0}$ be the set of lowest strata in $\mathcal{D}^{B}$. For $\mathcal{S}_{B}$ in $\mathcal{S}_{B, 0}$ set $U_{B}=\mathcal{M}_{\mathcal{S}_{B}}(B)$. Since $\mathcal{S}_{B}$ is minimal $U_{B}$ is compact and there exists $\epsilon_{\mathcal{S}_{B}}$ and a gluing map $G l_{\mathcal{S}_{B}}$ defined on the restriction of $\mathcal{L}_{\mathcal{S}_{B}, \epsilon_{\mathcal{S}_{B}}}$ to $U_{B}$. Furthermore, the minimal strata are isolated and for each $\mathcal{S}_{B} \in \mathcal{S}_{B, 0}$ we can choose a small enough $\epsilon_{B}$ so that the resulting gluing neighborhoods never intersect. Now let $\mathcal{S}_{P, 0,0, \mathcal{S}_{B}}$ be the set of lowest strata in $\mathcal{D}^{P} \cap S_{\pi_{*}}^{-1}\left(\mathcal{S}_{B}\right)$, where $\mathcal{S}_{B}$ is minimal. For any $\mathcal{S}_{P} \in \mathcal{S}_{P, 0,0, \mathcal{S}_{B}}$ set $U_{P}=\mathcal{M}_{\mathcal{S}_{P}}(P)$. Argumenting as above, there is $\epsilon_{\mathcal{S}_{P}}$ and $G l_{\mathcal{S}_{P}}$, with domain $\mathcal{L}_{\mathcal{S}_{P}, \epsilon_{\mathcal{S}_{P}}, U_{P}}^{*}$, such that $\bar{\pi} \circ G l_{\mathcal{S}_{P}}=G l_{\mathcal{S}_{B}} \circ \overline{\bar{\pi}} \overline{\mathcal{S}_{P}}$. Once again, we can choose the $\epsilon_{\mathcal{S}_{P}}$ such that $W_{\mathcal{S}_{P}} \cap W_{\mathcal{S}_{P}^{\prime}}=\emptyset$, for any two strata in $\mathcal{S}_{P, 0,0, \mathcal{S}_{B}}$.

Define inductively $\mathcal{S}_{B, k}$ as being the set of minimal strata in $\mathcal{D}^{B} \backslash \mathcal{S}_{B, k-1}$, and $\mathcal{S}_{P, k, m_{k}}$ as being the set of minimal strata in $\mathcal{D}^{P} \cap S_{\pi_{*}}^{-1}\left(\mathcal{S}_{B, k}\right) \backslash \mathcal{S}_{P, k, m_{k}-1}$. Suppose that each pairs $\left(U_{B}, \epsilon_{\mathcal{S}_{B}}\right)$ and $\left(U_{P}, \epsilon_{\mathcal{S}_{P}}\right)$, for $\mathcal{S}_{B} \in \mathcal{S}_{B, n}$ and $\mathcal{S}_{P} \in$ $\mathcal{S}_{P, n, m_{n}}$ with $n \leq k-1$ and $m_{n} \leq m_{k}-1$, have been chosen so that the induction holds. Set

$$
W_{\mathcal{S}_{B}, \mathcal{S}_{B}^{\prime}}:=G l_{\mathcal{S}_{B}, \mathcal{S}_{B}^{\prime}}\left(\mathcal{L}_{\mathcal{S}_{B}, \mathcal{S}_{B}^{\prime}, \epsilon_{\mathcal{S}_{B}}, U_{B}}\right)
$$

Then, for $\mathcal{S}_{B}^{\prime} \in \mathcal{S}_{B, k}$ we can choose a proper open subset $U_{B}^{\prime}$ such that $\left\{W_{\mathcal{S}_{B}, \mathcal{S}_{B}^{\prime}} \mid \mathcal{S}_{B} \prec \mathcal{S}_{B}^{\prime}\right\} \cup U_{B}^{\prime}$, is a covering for $\mathcal{M}_{\mathcal{S}_{B}^{\prime}}(B)$. Furthermore, there is $\epsilon_{\mathcal{S}_{B}^{\prime}}$ and a gluing map

$$
G l_{\mathcal{S}_{B}^{\prime}}: \mathcal{L}_{\mathcal{S}_{B}^{\prime}, \epsilon_{\mathcal{S}_{B}^{\prime}}^{\prime}, U_{B}^{\prime}}^{\rightarrow} \mathcal{M}_{0, l}\left(B, \sigma_{B}\right),
$$

and we can make sure that for all $\mathcal{S}_{B}^{\prime} \in \mathcal{S}_{B, k}$ and $\mathcal{S}_{B} \in \bigsqcup_{i=0}^{k} \mathcal{S}_{B, i}$, the intersection $W_{\mathcal{S}_{B}^{\prime}} \cap W_{\mathcal{S}_{B}}$ is empty unless $\mathcal{S}_{B} \prec \mathcal{S}_{B}^{\prime}$ (by choosing smaller $\epsilon_{\mathcal{S}_{B}^{\prime}}$ and $\epsilon_{\mathcal{S}_{B}}$ if necessary).

Since the gluings commute with the projection, it suffices to fix $\mathcal{S}_{B} \in \mathcal{S}_{B, k}$, and to apply the arguments given for $k=0$ to the elements of $\mathcal{S}_{P, k, m_{k}, \mathcal{S}_{B}}:=$ $\mathcal{S}_{P, k, m_{k}} \cap S_{\pi_{*}}^{-1}\left(\mathcal{S}_{B}\right)$. Set

$$
W_{\mathcal{S}_{P}, \mathcal{S}_{P}^{\prime}}:=G l_{\mathcal{S}_{P}, \mathcal{S}_{P}^{\prime}}\left(\mathcal{L}_{\mathcal{S}_{P}, \mathcal{S}_{P}^{\prime}, \epsilon_{\mathcal{S}_{P}}, U_{P}}\right)
$$

where $\mathcal{S}_{P}^{\prime}$ projects onto $\mathcal{S}_{B}$. Note that $W_{\mathcal{S}_{P}, \mathcal{S}_{P}^{\prime}}$ also projects onto $W_{\pi\left(\mathcal{S}_{B}\right), \pi\left(\mathcal{S}_{B}^{\prime}\right)}$. We can choose $U_{\mathcal{S}_{P}^{\prime}}$ such that $\left\{W_{\mathcal{S}_{P}, \mathcal{S}_{P}^{\prime}} \mid \mathcal{S}_{P} \prec \mathcal{S}_{P}^{\prime}\right\} \cup U_{P}^{\prime}$, covers $\mathcal{M}_{\mathcal{S}_{P}^{\prime}}(P)$, and 
for a well-chosen $\epsilon_{\mathcal{S}_{P}^{\prime}}$ we have a map $G l_{\mathcal{S}_{P}^{\prime}}$ whose image does not intersect the neighborhoods obtained so far unless it comes from a stratum $\mathcal{S}_{P}$ such that $\mathcal{S}_{P} \prec \mathcal{S}_{P}^{\prime}$.

A strata-covering gives an atlas. If the transition functions were to be smooth we would directly have a smooth orbifold structure on the considered moduli spaces. However, this may be hard to show and even not true in full generality. Instead, we show as in [3], Section 13.2, that for each stratum data there are charts $G l_{\mathcal{S}_{B}}$ such that the composition $G l_{\mathcal{S}_{B}} \circ G l_{\mathcal{S}_{B}, \mathcal{S}_{B}^{\prime}}^{-1}$ is differentiable for every $\mathcal{S}_{B}^{\prime} \prec \mathcal{S}_{B}$, which provides, not canonically, a differentiable atlas.

Lemma 6.6. There are strata-coverings $\left(U_{P}, \epsilon_{\mathcal{S}_{P}}\right)$ and $\left(U_{B}, \epsilon_{\mathcal{S}_{B}}\right)$, and gluing maps, $G l_{\mathcal{S}_{P}}$ and $G l_{\mathcal{S}_{B}}$, compatible with $\bar{\pi}_{\mathcal{S}_{P}}$, such that for every stratum data $\mathcal{S}_{P}$ and $\mathcal{S}_{B}$, the maps $G l_{\mathcal{S}_{P}}$ and $G l_{\mathcal{S}_{B}}$ coincide with any other gluing maps

$$
G l_{\mathcal{S}_{P}}^{\prime}=G l_{\mathcal{S}_{P}^{\prime}} \circ G l_{\mathcal{S}_{P}^{\prime}, \mathcal{S}_{P}}^{-1} \quad \text { and } \quad G l_{\mathcal{S}_{B}}^{\prime}=G l_{\mathcal{S}_{B}^{\prime}} \circ G l_{\mathcal{S}_{B}^{\prime}, \mathcal{S}_{B}}^{-1},
$$

where $\mathcal{S}_{P}^{\prime} \prec \mathcal{S}_{P}$ and $\mathcal{S}_{B}^{\prime} \prec \mathcal{S}_{B}$.

Proof. The proof is again by induction. Let $\mathcal{S}_{B, k}$ and $\mathcal{S}_{P, k, m_{k}}$ as in lemma 6.5. We see that the result holds for $\mathcal{S}_{B, 0}$ and $\mathcal{S}_{P, 0,0}$. Suppose it is true for all $\mathcal{S}_{B} \in \mathcal{S}_{B, n}$ and $\mathcal{S}_{P} \in \mathcal{S}_{P, n, m_{n}}$ such that $n \leq k-1$ and $m_{n} \leq m_{k}-1$. Let $\mathcal{S}_{B} \in \mathcal{S}_{B, k}$ and set $W_{\mathcal{S}_{B}}:=\cup_{\mathcal{S}_{B}^{\prime} \prec \mathcal{S}_{B}} W_{\mathcal{S}_{B}^{\prime}, \mathcal{S}_{B}}$. Let $G l_{\mathcal{S}_{B}}^{\prime}\left(\mathcal{S}_{B}^{\prime}\right)$ be the gluing map induced by $\mathcal{S}_{B}^{\prime} \prec \mathcal{S}_{B}$. Recall that this map is defined above $W_{\mathcal{S}_{B}^{\prime}, \mathcal{S}_{B}}$. We must show that

$$
G l_{\mathcal{S}_{B}}^{\prime}\left(\mathcal{S}_{B}^{\prime}\right)=G l_{\mathcal{S}_{B}}^{\prime}\left(\mathcal{S}_{B}^{\prime \prime}\right),
$$

on $W_{\mathcal{S}_{B}^{\prime}, \mathcal{S}_{B}} \cap W_{\mathcal{S}_{B}^{\prime \prime}, \mathcal{S}_{B}}$. But this latter intersection is non-empty if and only if $\mathcal{S}_{B}^{\prime \prime} \prec \mathcal{S}_{B}^{\prime}$. But from the induction, $G l_{\mathcal{S}_{B}^{\prime}}^{\prime}\left(\mathcal{S}_{B}^{\prime \prime}\right)=G l_{\mathcal{S}_{B}^{\prime}}$ on $W_{\mathcal{S}_{B}^{\prime \prime}, \mathcal{S}_{B}^{\prime}} \cap U_{B}^{\prime}$. Thus

$$
\begin{aligned}
G l_{\mathcal{S}_{B}}^{\prime}\left(\mathcal{S}_{B}^{\prime \prime}\right) & =G l_{\mathcal{S}_{B}^{\prime \prime}} \circ G l_{\mathcal{S}_{B}^{\prime \prime}, \mathcal{S}_{B}}^{-1} \\
& =G l_{\mathcal{S}_{B}^{\prime \prime}} \circ G l_{\mathcal{S}_{B}^{\prime \prime}, \mathcal{S}_{B}^{\prime}}^{-1} \circ G l_{\mathcal{S}_{B}^{\prime \prime}, \mathcal{S}_{B}^{\prime}} \circ G l_{\mathcal{S}_{B}^{\prime \prime}, \mathcal{S}_{B}}^{-1} \\
& =G l_{\mathcal{S}_{B}^{\prime}} \circ G l_{\mathcal{S}_{B}^{\prime \prime}, \mathcal{S}_{B}^{\prime}}^{\prime} \circ G l_{\mathcal{S}_{B}^{\prime \prime}, \mathcal{S}_{B}}^{-1},
\end{aligned}
$$

giving (6.32). As a result, we obtain a gluing map $G l_{\mathcal{S}_{B}}^{\prime}$ defined on $W_{\mathcal{S}_{B}}$. Now, given a gluing map $G l_{\mathcal{S}_{B}}^{\prime \prime}$ on $U_{B}$, we derive a third map $G l_{\mathcal{S}_{B}}$, which is obtained as an interpolation between $G l_{\mathcal{S}_{B}}^{\prime}$ and $G l_{\mathcal{S}_{B}}^{\prime \prime}$ using a cut-off function. This ends the induction for $\overline{\mathcal{M}}_{0, l}\left(B, \sigma_{B}\right)$.

We explain in details how to interpolate $G l_{\mathcal{S}_{B}}^{\prime}$ and $G l_{\mathcal{S}_{B}}^{\prime \prime}$. By definition $G l_{\mathcal{S}_{B}}^{\prime}\left(\mathcal{S}_{B}^{\prime}\right)$ is of type-2 with domain:

$$
W_{\mathcal{S}_{B}^{\prime}, \mathcal{S}_{B}}=G l_{\mathcal{S}_{B}^{\prime}, \mathcal{S}_{B}}\left(\mathcal{L}_{\mathcal{S}_{B}^{\prime}, \mathcal{S}_{B}, \epsilon_{\mathcal{S}_{B}^{\prime}}, U_{B}^{\prime}}\right) \equiv W_{\mathcal{S}_{B}^{\prime}, \mathcal{S}_{B}}\left(\epsilon_{\mathcal{S}_{B}^{\prime}}\right)
$$


For admissible $G l_{\mathcal{S}_{B}^{\prime}, \mathcal{S}_{B}}$, the associated chart data are given by the triple $\left(V:=\operatorname{pgl}\left(\mathcal{L}_{\mathcal{S}_{B}^{\prime}, \mathcal{S}_{B}, \epsilon_{\mathcal{S}_{B}^{\prime}}}\right), \phi, F\right)$ where:

$$
F: V \rightarrow \mathcal{M}_{\mathcal{S}_{B}}(B), \quad\left(u_{B, \rho}, \mathbf{j}_{\rho}\right) \mapsto \exp _{u_{B, \rho}} Q_{u_{B, \rho}}^{B} f_{\mathcal{S}_{B}}\left(u_{B}, \mathbf{j}, \rho\right) .
$$

Consider a function

$$
\nu: \mathcal{L}_{\mathcal{F}_{B}\left(\mathcal{S}_{B}^{\prime}\right), \mathcal{F}_{B}\left(\mathcal{S}_{B}\right), \epsilon_{\mathcal{S}_{B}^{\prime}}} \rightarrow \mathbb{R}, \quad \nu(\mathbf{j}, \rho) \mapsto \begin{cases}0 & \text { si }|\rho| \leq 0.5 \epsilon_{\mathcal{S}_{B}^{\prime}}, \\ 1 & \text { si }|\rho| \geq 0.75 \epsilon_{\mathcal{S}_{B}^{\prime}}^{\prime}\end{cases}
$$

We now "glue" the domains of the chart data for $G l_{\mathcal{S}_{B}}^{\prime \prime}$ and $G l_{\mathcal{S}_{B}}^{\prime}\left(\mathcal{S}_{B}^{\prime}\right)$, i.e., we glue $U_{B}$ and $V$. The new domain

$$
V^{\prime}:=\operatorname{Im}\left(\exp _{u_{B, \rho}}\left(\nu(\mathbf{j}, \rho) Q_{u_{B, \rho}}^{B} f_{\mathcal{S}_{B}}\left(u_{B}, \mathbf{j}, \rho\right)\right)\right)
$$

coincides with $V$ for $|\rho| \leq 0.5 \epsilon_{\mathcal{S}_{B}^{\prime}}$, and with $U_{\mathcal{S}_{B}^{\prime}}$ when $|\rho| \geq 0.75 \epsilon_{\mathcal{S}_{B}^{\prime}}$. We can make sure that the pair, $\left(V^{\prime},\left\{Q_{u_{B}}^{B} \mid u_{B} \in V^{\prime}\right\}\right)$, satisfies hypothesis (6.1) since $V^{\prime}$ is a uniform deformation between $V$ and $U_{B}$ ( $\nu$ does not depend on $u_{B}$ ). Denote by $G l_{\mathcal{S}_{B}}$ the gluing map arising from the pair $\left(V^{\prime},\left\{Q_{u_{B}}^{B} \mid u_{B} \in V^{\prime}\right\}\right)$. Then, $G l_{\mathcal{S}_{B}}$ coincides with $G l_{\mathcal{S}_{B}}^{\prime}\left(\mathcal{S}_{B}^{\prime}\right)$ on $V \cap V^{\prime}=W_{\mathcal{S}_{B}^{\prime}, \mathcal{S}_{B}}\left(0.5 \epsilon_{\mathcal{S}_{B}^{\prime}}\right)$, and with $G l_{\mathcal{S}_{B}}^{\prime \prime}$ on $U_{B} \cap V^{\prime}$. In particular, this map extends to $U_{B} \backslash W_{\mathcal{S}_{B}^{\prime}, \mathcal{S}_{B}}\left(\epsilon_{\mathcal{S}_{B}^{\prime}}\right)$. Regarding $\overline{\mathcal{M}}_{0, l}(P, \sigma)$ we proceed similarly. Fixing $\mathcal{S}_{B} \in \mathcal{S}_{B, k}$, and applying the same arguments as above to the elements $\mathcal{S}_{P} \in \mathcal{S}_{P, k, m_{k}, \mathcal{S}_{B}}$, ends the proof.

\section{References}

[1] R. Bott and W. Tu. Loring, Differential forms in algebraic topology, Vol. 82, Graduate Texts in Mathematics. Springer-Verlag, New York, 1982.

[2] O. Buse, Relative family Gromov-Witten invariants and symplectomorphisms, 2001, arXiv: org: math/0110313.

[3] B. Chen and A.-M. Li, Symplectic virtual localization of Gromov-Witten invariants, 2006, arXiv:org:math/0610370.

[4] K. Costello, The $A$-infinity operad and the moduli space of curves, 2004, arXiv: org: math/0402015.

[5] V. Guillemin, E. Lerman and Sh. Sternberg, Symplectic fibrations and multiplicity diagrams, Cambridge University Press, Cambridge, 1996.

[6] J. Hu, T-J. Li and Y. Ruan, Birational cobordism invariance of uniruled symplectic manifolds, 2006, arXiv:org:math/0611592.

[7] Ch. Hummel, Gromov's compactness theorem for pseudo-holomorphic curves, Vol. 151, Progress in Mathematics, Birkhäuser-Verlag, Basel, 1997.

[8] C. Hyvrier, Invariants de Gromov-Witten et fibrations hamiltoniennes, Dissertation de Thèse, 2008.

[9] M. Kontsevich and Yu. Manin, Quantum cohomology of a product, Invent. Math. 124(1-3) (1996), 313-339. With an appendix by R. Kaufmann. 
[10] F. Lalonde and D. McDuff, Symplectic structures on fiber bundles Topology 42(2) (2003), 309-347.

[11] F. Lalonde, D. McDuff and L. Polterovich, Topological rigidity of Hamiltonian loops and quantum homology, Invent. Math. 135(2) (1999), 369-385.

[12] H.-V. Le and K. Ono, Parameterized Gromov-Witten invariants and topology of symplectomorphism groups, 2007, arXiv:org:0704.3899.

[13] J. Li and G. Tian, Virtual moduli cycles and Gromov-Witten invariants of general symplectic manifolds, in Topics in symplectic 4-manifolds (Irvine, CA, 1996), First International Press Lecture Series, I, Int. Press, Cambridge, MA, 1998, 47-83.

[14] G. Liu, Associativity of quantum multiplication, Comm. Math. Phys. 191(2) (1998), 265-282.

[15] D. McDuff, Quantum homology of fibrations over $S^{2}$, Int. J. Math. 11(5) (2000), $665-721$.

[16] D. McDuff and D. Salamon, Introduction to symplectic topology, Oxford Mathematical Monographs, Clarendon Press, Oxford University Press, New York, 2nd ed., 1998.

[17] D. McDuff and D. Salamon, J-holomorphic curves and symplectic topology, Vol. 52, American Mathematical Society Colloquium Publications, American Mathematical Society, Providence, RI, 2004.

[18] D. McDuff, Examples of symplectic structures, Invent. Math. 89(1) (1987), 13-36.

[19] Y. Ruan, Topological sigma model and Donaldson-type invariants in Gromov theory, Duke Math. J. 83(2) (1996), 461-500.

[20] Y. Ruan, Virtual neighborhoods and pseudo-holomorphic curves, in Proceedings of 6th Gökova Geometry-Topology Conf., Vol. 23, 1999, 161-231.

[21] Y. Ruan and G. Tian, A mathematical theory of quantum cohomology, J. Differ. Geom. 42(2) (1995), 259-367.

[22] P. Seidel, $\pi_{1}$ of symplectic automorphism groups and invertibles in quantum homology rings, Geom. Funct. Anal. 7(6) (1997) 1046-1095.

[23] J-C. Sikorav, The gluing construction for normally generic J-holomorphic curves, in Symplectic and contact topology: interactions and perspectives (Toronto, ON/Montreal, QC, 2001), Vol. 35, Fields Inst. Commun., Amer. Math. Soc., Providence, RI, 2003, 175-199.

[24] A. Zinger, Enumerative vs. symplectic invariants and obstruction bundles, J. Symplectic Geom. 2(4) (2004), 445-543.

UNIVERSITY OF UPPSALA

Mathematics Department

Box 480

75106 UPPSALA

SWEDEN

E-mail address: clement.hyvrier@math.uu.se

Received: 06/21/2010, accepted 03/24/2011

I would like to thank François Lalonde and Shengda Hu, for being so generous of their time and support, and for the fruitful discussions I had with them. I should also thank Dusa McDuff for bringing symplectic uniruledness to my mind. Also, many thanks to Frédéric Bourgeois and his corrections and suggestions regarding this work. I should also thank Rémi Leclercq and Liam Watson for their useful comments. Finally, I am grateful to the referee for his suggestions and comments that helped improve the manuscript. 